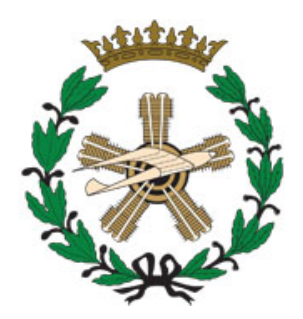

\title{
GLOBAL INSTABILITY ANALYSIS AND CONTROL OF THREE-DIMENSIONAL LONG OPEN CAVITY FLOW
}

Doctoral Thesis

By

Qiong Liu

Aeronautical Engineer 

ESCUELA TÉCNICA SUPERIOR DE INGENIEROS AERONÁUTICOS

UNIVERSIDAD POLITÉCNICA DE MADRID

Doctoral Thesis

\title{
GLOBAL INSTABILITY ANALYSIS AND CONTROL OF THREE-DIMENSIONAL LONG OPEN CAVITY FLOW
}

\author{
by \\ Qiong Liu \\ Aeronautical Engineer \\ Advisors \\ Vassilis Theofilis and Francisco Gómez Carrasco
}

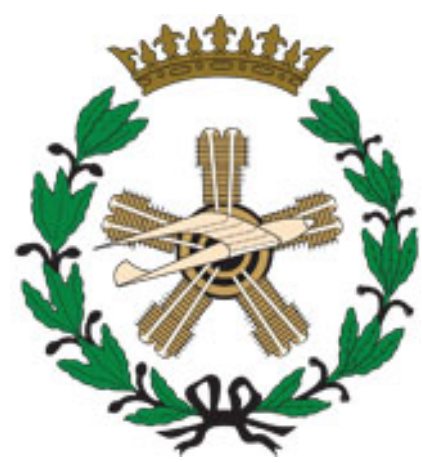

MAdrid, February 2016 
This page intentionally left blank 


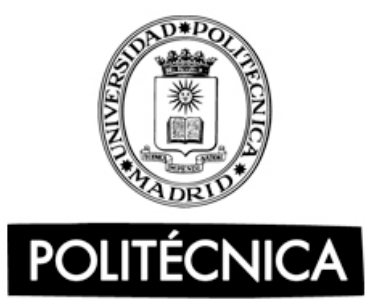

Tribunal nombrado por el Sr. Rector Magfco. de la Universidad Politécnica de Madrid, el día. de. de $20 \ldots$

Presidente:

Vocal:

Vocal:

Vocal:

Secretario:

Suplente:

Suplente:

Realizado el acto de defensa y lectura de la Tesis el día de. de $20 \ldots$ en la E.T.S.I. /Facultad.

Calificación

EL PRESIDENTE

LOS VOCALES 
This page intentionally left blank 
Have no fear of perfection; you'll never reach it.

- Marie Curie 


\section{Abstract}

The three-dimensional wall-bounded open cavity may be considered as a simplified geometry found in industrial applications such as leading gear or slotted flats on the airplane. Understanding the three-dimensional complex flow structure that surrounds this particular geometry is therefore of major industrial interest.

At the light of the remarkable former investigations in this kind of flows, enough evidences suggest that the lateral walls have a great influence on the flow features and hence on their instability modes. Nevertheless, even though there is a large body of literature on cavity flows, most of them are based on the assumption that the flow is two-dimensional and spanwise-periodic. The flow over realistic open cavity should be considered. This thesis presents an investigation of three-dimensional wall-bounded open cavity with geometric ratio 6:2:1. To this aim, three-dimensional Direct Numerical Simulation (DNS) and global linear instability have been performed.

Linear instability analysis reveals that the onset of the first instability in this open cavity is around $R e_{c r} \sim 1080$. The three-dimensional shear layer mode with a complex structure is shown to be the most unstable mode. It is noteworthy that the flow pattern of this high-frequency shear layer mode is similar to the observed unstable oscillations in supercritical unstable case.

DNS of the cavity flow carried out at different Reynolds number from steady state until a nonlinear saturated state is obtained. The comparison of time histories of kinetic energy presents a clearly dominant energetic mode which shifts between low-frequency and highfrequency oscillation. A complete flow patterns from subcritical cases to supercritical case has been put in evidence. The flow structure at the supercritical case $R e=1100$ resembles typical wake-shedding instability oscillations with a lateral motion existed in the subcritical cases. Also, This flow pattern is similar to the observations in experiments.

In order to validate the linear instability analysis results, the topology of the composite flow fields reconstructed by linear superposition of a three-dimensional base flow and its leading three-dimensional global eigenmodes has been studied. The instantaneous wall streamlines of those composited flows display distinguish influence region of each eigenmode. Attention has been focused on the leading high-frequency shear layer mode; the composite flow fields have been fully recognized with respect to the downstream wave shedding. The three-dimensional shear layer mode is shown to give rise to a typical wake-shedding instability with a lateral motions occurring downstream which is in good agreement with the experiment results. Moreover, the spanwise-periodic, open cavity with the same length to depth ratio has been also studied. The most unstable linear mode is different from the real three-dimensional cavity flow, because of the existence of the side walls.

Structure sensitivity of the unstable global mode is analyzed in the flow control context. The adjoint-based sensitivity analysis has been employed to localized the receptivity region, where the flow is more sensible to momentum forcing and mass injection. Because 
of the non-normality of the linearized Navier-Stokes equations, the direct and adjoint field has a large spatial separation. The strongest sensitivity region is locate in the upstream lip of the three-dimensional cavity. This numerical finding is in agreement with experimental observations. Finally, a prototype of passive flow control strategy is applied. 


\section{Acknowledgements}

To my parents

In the course of this work I have been supported by many people. I want to sincerely thank all of them, without who the work would have not been so fulfilling and rewarding.

Firstly, I want to thank my advisor Professor Vassilis Theofilis. He gave me the opportunity to begin learn working under his guidance. Thanks to this, I decided to start my research career in the field of fluid mechanics, specifically in the study of flow instabilities. This thesis is the outcome of his support and advice during 4 and half years. I also want to shown my gratefulness to my co-advisor Dr. F. Gómez, who works in Monash University. He teaches me a lot, from the thought of doing research to the paper writing. He squeezes his precious time to have a international discussion weekly.

In the second year of my study, Vassilis Theofilis supplied an opportunity for me to visiting Professor M.A.Faraco de Medeiros in University of São Paulo. In the shortly three month, Professor M.A.Faraco de Medeiros patiently guided my research and made me confident, evoked my desire to study fluid dynamics. I would like to appreciate Professor M.A. Faraco de Medeiros. During the study in São Carlos, my colleague and friend Dr. D. Rodríguez is a great help. It has been a pleasure to collaborate with Dr. Melina for the dynamic mesh technique in OpenFOAM. At the same time, I met a lot of sincere friends in Brazil, Dr. Elmer Mateus Gennaro, Daniel Souza, Andres Martinez, Leandro Bergama, a kindly appreciation for all of them.

I want to show my gratitude to all the people present in the day-day work, that provided a great working environment. Dr. José Miguel Pérez is the first friend I met in Madrid, he's humor and kind heart helped me when I was suddenly in a unfamiliar situation. Kindly appreciations to My friend Dr. Pedro Paredes, he helped my programming my first Matrix-forming code and encouraged me to make a good job, I have a chance to recognize his fiancée, Maria José, I learned Spanish cuisine from her. My kind-hearted office mate Juan Angel Tendero, Wei He, Dong SiWei and Soledad Le Clainche are great appreciated.

During the work, I recklessly asked help from people who I never have a chance to see face to face. Thanks to Dr. Jean-Christophe Loiseau and Mattias Brynjell-rahkola for the unselfish instruction in Nek5000.

Thanks to my private friend Yu JianGang in RWTH Aachen University, the communication with him for the Ph.D life really helps me in hard time. He lent me his computational resource when my daedalus had a fatal problem.

I am willing to express myheartful appreciation to my parents, Yu HuYang, my older brother Liu YuZhou, my sister Cui LiPing, my dear nephew Liu YiXuan. Your endless support and confidence are great treasure in my life. 


\section{Contents}

$\begin{array}{ll}\text { Abstract } & \text { iii }\end{array}$

$\begin{array}{ll}\text { Acknowledgements } & \text { V }\end{array}$

$\begin{array}{ll}\text { Contents } & \text { vii }\end{array}$

1 Introduction $\quad 1$

1.1 Novelty and Overview . . . . . . . . . . . . . . . . . . . 6

1.2 Publications ......................... 7

2 Hydrodynamic Instability $\quad 9$

2.1 Navier-Stokes equations . . . . . . . . . . . . . . . . . . . . . 9

2.2 Instability analysis theory . . . . . . . . . . . . . . . . . . 10

2.2.1 BiGlobal stability analysis . . . . . . . . . . . . . . 12

2.2.2 TriGlobal stability analysis . . . . . . . . . . . . . . 12

2.3 Determination of the Instability Core: Structure Sensitivity . . . . . . . . 13

3 Numerical Solution of the Eigenvalue Problem 15

3.1 Direct and adjoint linearized Navier-Stokes equations . . . . . . . . . . . . . 16

3.2 Residual Algorithm . . . . . . . . . . . . . . . . . . . . . 18

3.3 Time-stepping method implemented in Nek5000 . . . . . . . . . . . . . . . . 22

3.4 Steady state solution . . . . . . . . . . . . . . . . . . . 24

4 Validations $\quad 27$

4.1 Validation in OpenFoam . . . . . . . . . . . . . . . . . . 27

4.1.1 Two-dimensional open cavity flow . . . . . . . . . . . . 27

4.1.2 Spanwise periodic open cavity flow . . . . . . . . . . . . . 30

4.2 Validation in Nek5000 . . . . . . . . . . . . . . . . . . . . . 33

4.2.1 Two-dimensional lid-driven cavity validation . . . . . . . . . . 33

4.2.2 Two-dimension flow over cylinder . . . . . . . . . . . . . 38

4.2.3 Square open cavity with $L: \Lambda: D=1: 1: 1$. . . . . . . . . . . . . . . . . 43

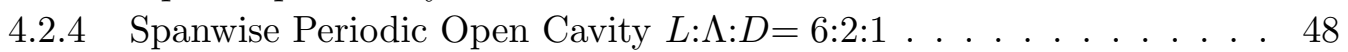

4.2.5 Three-dimensional lid-driven cavity validation ............ 51

5 Three-dimensional wall-bounded open cavity flow 55

5.1 Model description and parameters . . . . . . . . . . . . . . 55

5.2 Boundary conditions and initial conditions . . . . . . . . . . . . . 55

5.2 .1 Domain and grid validations . . . . . . . . . . . . . 57

5.3 Tri-Global linear instability of three-dimensional open cavity flow . . . . . . 61

5.3.1 Case 1: Re $=900$. . . . . . . . . . . . . . 61 
5.3 .2 Case $2: R e=950 \ldots \ldots \ldots \ldots 6 \ldots$

5.3 .3 Case $3: R e=1000 \ldots \ldots \ldots \ldots 70 \ldots \ldots$

5.3 .4 Case $4: R e=1050 \ldots \ldots \ldots \ldots$. . . . . . . . . . . . . . .

5.4 Critical Reynolds number evaluation . . . . . . . . . . . . . . 82

5.5 Topological changes exerted by the global modes . . . . . . . . . . 83

5.6 Sensitivity sensitivity . . . . . . . . . . . . . . . . 85

5.7 Eigenvalue sensitivity to the size of computational domain . . . . . . . . 86

5.8 Nonlinear evolution of supercritical flow . . . . . . . . . . . 88

6 Attempts for the cavity flow control $\quad 93$

6.1 Passive cavity flow control via square cylinder . . . . . . . . . . . . 93

$\begin{array}{lll}7 & \text { Final Remarks } & 99\end{array}$

$\begin{array}{ll}\text { A Adjoint equations } & 103\end{array}$

A.0.1 Variables and inner products . . . . . . . . . . . . . . . 103

A.0.2 Operators and PDEs . . . . . . . . . . . . . . . . 103

A.0.3 Boundary and initial conditions . . . . . . . . . . . . . . . . 104

$\begin{array}{ll}\text { B Chebyshev Polynomial and Staggered grid } & 107\end{array}$ 


\section{List of Figures}

1.1 Sketches of global instability analysis examples . . . . . . . . . . . . 2

2.1 Sketches of typical impulse responses . . . . . . . . . . . . . . . . 10

2.2 Sketches of global instability analysis examples . . . . . . . . . . . . . . 13

3.1 Temporal exponential transformation $[52] \ldots \ldots \ldots \ldots$

4.1 Two-dimensional open cavity mesh. . . . . . . . . . . . . . . . . . . . 29

4.2 Residual of kinetic energy. . . . . . . . . . . . . . . . . . 30

4.3 The real part of direct and adjoint eigenmodes at $R e=1400$. . . . . . . 31

4.4 The real part of direct and adjoint eigenmodes at $R e=1900 . \ldots$. . . . . . 31

4.5 Structure functions of $2 \mathrm{D}$ open cavity. . . . . . . . . . . . . . . . 32

4.6 Direct module, adjoint module and sensitivity region of 3D open cavity. . . 33

4.7 (a) Lid-driven cavity mesh based on the spectral element method. (b) Temporal evolution of the velocity residual of the $2 \mathrm{D}$ lid-driven cavity at

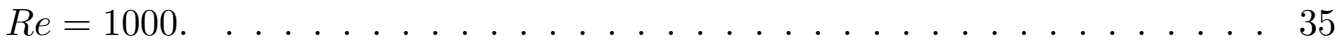

4.8 The steady state stable base flow of lid-driven cavity at $R e=2000 \ldots$. . . . 35

4.9 The comparison of the spectrum of two-dimensional lid-driven cavity at $R e=2000$ calculated by matrix-forming method $(\mathrm{EVP}, \bullet)$ and current method matrix-free method (TS, $\square$ ). The matrix-forming results refers to the private communication with J.M. Pérez. . . . . . . . . . . . . . . 36

4.10 Eigenmodes of the two-dimensional regularized lid-driven cavity ( LCD) obtained using time-stepping method at $R e=2000$. The solid iso-lines $(-)$ represent positive eigenmodes and the dash iso-line (--) label negative eigenmodes. 10 contour-level of the iso-lines are plotted for the values $\hat{u}(x, y)=[-1,1]$ and $\hat{v}(x, y)=[-0.7,0.65]$, both $\hat{u}(x, y)$ and $\hat{v}(x, y)$ normalized by maximum value of $\hat{u}(x, y) \ldots \ldots \ldots 37$

4.11 Schematic description of the $2 \mathrm{D}$ cylinder. . . . . . . . . . . 38

4.12 (a) mesh of the cylinder; (b) mesh detail near the cylinder . . . . . . . . . 39

4.13 The snapshot of the time-periodic solution of (a) $u(x, y)$ and (b) $v(x, y)$ at $R e=40$; The snapshot of the time-periodic solution of (c) $u(x, y)$ and (d) $v(x, y)$ at $R e=50$; The snapshot of the time-periodic solution of (e) $u(x, y)$ and (f) $v(x, y)$ at $R e=100$; The steady unstable base flow (g) $u(x, y)(\mathrm{h}) v(x, y)$ of $R e=100$ from selective frequency damping method. . 40

4.14 (a) Amplification rate and (b) Strouhal number dependence on Re from the previous research of Giannetti and Luchini [49], $\square$ represent the results from current research. . . . . . . . . . . . . . . . . . 4 4 41 
4.15 (a), (b) The leading unstable eigenmode $\lambda=-0.0291+i \cdot 0.7485$ of cylinder at $R e=40$. (c), (d) The leading unstable eigenmode $\lambda=0.01611+i \cdot 0.7452$ of cylinder at $R e=50$. (e), (f) The leading unstable eigenmode $\lambda=$ $0.1253+i \cdot 0.7310$ of cylinder at $R e=100 \ldots \ldots$. . . . . . . . 42

4.16 Schematic description of the square open cavity. . . . . . . . . . . . . 43

4.17 Mesh information of the square open cavity. . . . . . . . . . . . . . 43

4.18 (a), (b) 2D steady unstable base flow of square open cavity ( $L: D: \Lambda=1$ : $1: 1)$ flow at $R e=4140 \ldots \ldots \ldots$. . . . . . . . . . . . 44

4.19 Comparison of eigenvalue spectrum of square open cavity at $R e=4140$ and $\beta=22$ from current results $(\square)$ and results from Citro et al. [27] $(\bullet) . \quad$. . . 45

4.20 Contour plots of the direct modes: (a) mode I: $0.2380+i \cdot 0.000$, (b)mode II $: 0.2243+i \cdot 0.3005,(\mathrm{c})$ mode III: $0.1811+i \cdot 0.6036,(\mathrm{~d})$ mode IV: $0.1176+$ $i \cdot 0.9134$.

4.21 Real part of the eigenfunction velocity field of the spanwise periodic square open cavity at $R e=4140$. Eigenfunctions normalized $\max (\hat{u})$. (a)-(c)mode I: $0.2380+i \cdot 0.000 ;$ (d)-(f)mode II $: 0.2243+i \cdot 0.3005$; (g)-(i)mode III: $0.1811+i \cdot 0.6036 ;(\mathrm{g})-(\mathrm{i})$ mode IV: $0.1176+i \cdot 0.9134$. The iso-surface level are: $\hat{v}(x, y, z), \hat{v}(x, y, z), \hat{w}(x, y, z)= \pm 0.15 \ldots \ldots \ldots \ldots$

4.22 The instantaneous numerical schilieren of (a) $p= \pm 0.4$ and (b) $\omega_{z}= \pm 8$ from DNS. The steady state of (c) $p= \pm 0.04$ and (b) vorticity contours $\omega_{z}= \pm 5$ using SFD. . . . . . . . . . . . . . . . 4 48

4.23 Spectrum of spenwise periodic open cavity $\mathrm{L}: \Lambda: \mathrm{D}=6: 2: 1$ at $R e=1000 \ldots \quad \ldots 49$

4.24 Real part of the eigenfunctions velocity field of the $3 \mathrm{D}$ cavity at $R e=1000$, eigenfunctions normalized with $\max (\hat{u}) . \ldots . . . . . . . .50$

4.25 Geometrical description of the problem and main flow features from Gómez et al. [54] . . . . . . . . . . . . . . . . . . . . . 5 51

4.26 Normal velocity on the centerlines $(0.5, y, 0.5)$ and $(x, 0.5,0.5)$ in the cubic cavity for $L: W: D=1: 1: 1$ using a resolution $N_{x} \times N_{y} \times N_{z}=96 \times 96 \times 96$ at $R e=1000$. The symbol $(\diamond)$ represents the results of Albensoeder and Kuhlmann [2].

4.27 Spectrum of cubic lid-driven cavity at $R e=1000$ calculated by Giannetti et al. [51](GLM, × ) and Gómez et al. [54](FVM, •) and current method

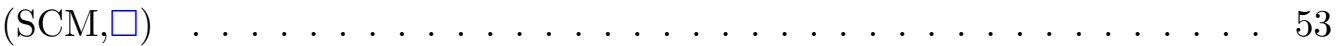

4.28 (a)-(c)mode I: $-0.1353+i \cdot 0.299 \hat{u}(x, y, z)= \pm 0.6, \hat{v}(x, y, z)= \pm 0.8, \hat{w}(x, y, z)=$ \pm 0.8 (d)-(f)mode II :-0.1304+i.0.488 $\hat{u}(x, y, z)= \pm 0.6, \hat{v}(x, y, z)= \pm 0.6, \hat{w}(x, y, z)=$ \pm 1 (g)(i)mode III: $-0.1372+i \cdot 0.000, \hat{v}(x, y, z)= \pm 0.8, \hat{v}(x, y, z)= \pm 0.8, \hat{w}(x, y, z)=$

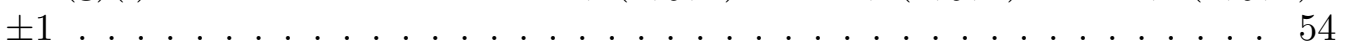

5.1 Computational configuration of full 3D open cavity . . . . . . . . . . 56

5.2 Spatial visualization of $u$ and $w$ in three spanning computational domain

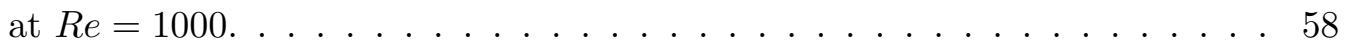

5.3 The grid detail for computation . . . . . . . . . . . . . . . 60

5.4 Validation of the time history of the streamwise velocity $u(x, y, z)$ in the center of the cavity $(3,0,1)$ at $R e=1000$ with three different polynomial orders. . . . . . . . . . . . . . . . . . 6 60

5.5 Time trace of streamwise velocity $u(3,0,1)$ and the details of the signal for the cuboid cavity at $R e=900$. 
5.6 (a) Time evolution of kinetic energy residual of wall-bounded three-dimensional open cavity at $R e=900$. (b) Eigen-spectrum of obtained global modes at

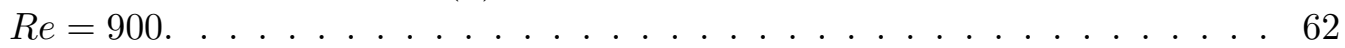

$5.73 \mathrm{D}$ visualization of cavity flow at $R e=900 \ldots \ldots \ldots \ldots$. . . . . . . . 62

5.8 Eigen-spectrum of obtained global modes at $R e=900 \ldots \ldots$. . . . . . . 64

5.9 Real part of the eigenfunction velocity field of the cuboid cavity at $R e=900$, Eigenfunctions normalized with $\max (\hat{u})$. From upper to lower are corresponding to the eigenfunction of eigenmodes listed in table 5.5. Isosurfaces $\hat{u}= \pm 0.1, \hat{v}, \hat{w}= \pm 0.05$. (left): $\hat{u},($ middle) $: \hat{v}$ and $($ right $): \hat{w} . \ldots \ldots 65$

5.10 Time trace of streamwise velocity $u(3.0,0.0,1.0)$ and the details of the signal for the cuboid cavity at $R e=950$.

5.11 (a) Contour of the spanwise velocity $w(x, y, z)$ of the cuboid cavity at $R e=950$, isosurface level are $w(x, y, z)= \pm 0.01$. (b) Grey isosurfaces are streamwise vorticity $\omega_{x}= \pm 0.15$, the blue isosurfaces are negative stream-

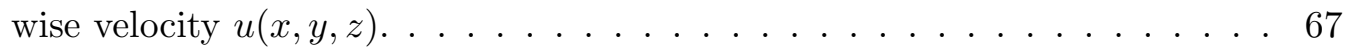

5.12 Eigen-spectrum of cuboid cavity at $R e=950 \ldots \ldots$. . . . . . . 67

5.13 Real part of the eigenfunction velocity field of the cuboid cavity at $R e=$ 950, Eigenfunctions normalized with $\max (\hat{u})$. From upper to lower are corresponding to the eigenfunction of mode 1-6. Isosurfaces $\hat{u}= \pm 0.1$, $\hat{v}, \hat{w}= \pm 0.05$. (left): $\hat{u},($ middle $): \hat{v}$ and (right) $: \hat{w} . \ldots \ldots . \ldots 69$

5.14 Time trace of kinetic energy $(E / U)$ and the details of the signal for the cuboid cavity at $R e=1000 \ldots \ldots \ldots \ldots \ldots$. . . . . . . . . . . . .

5.15 The shear layer vorticity thickness $\delta_{\omega}$ along the cuboid cavity at $R e=$ 1000(०) and reference date from Rowley et al. $[101](\nabla)$ at $M a=0.6$ of the compressible cavity flow. . . . . . . . . . . . . . . . 72

5.16 (a) Time evolution of the kinetic energy residual of the cuboid cavity at $R e=1000$, (b) Power spectral of kinetic energy at time domain [200,480], only the most energetic frequency are captured. . . . . . . . . . . 72

5.17 (a) Contour of the spanwise velocity $w(x, y, z)$ of the cuboid cavity at $R e=1000$, isosurface level are $w(x, y, z)= \pm 0.01$. (b) Grey isosurfaces are streamwise vorticity $\omega_{x}= \pm 0.15$, the blue isosurfaces are negative streamwise velocity $\omega_{x}(x, y, z) \ldots \ldots \ldots \ldots \ldots \ldots$

5.18 Spectrum of open cavity at $R e=1000 \ldots \ldots \ldots$. . . . . . . . . . . 74

5.19 Real part of the eigenfunction velocity field of the three-dimensional open cavity at $R e=1000$, Eigenfunctions normalized with $\max (\hat{u})$. From upper to lower are corresponding to the eigenfunctions of mode 1-6. Isosurfaces $\hat{u}= \pm 0.1, \hat{v}, \hat{w}= \pm 0.05$. (left) $: \hat{u},($ middle $): \hat{v}$ and $($ right $): \hat{w} . \ldots . \ldots 75$

5.20 Time trace of kinetic energy $E / U$ and the details of the signal for the cuboid

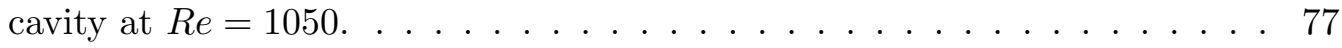

5.21 (a) Time evolution of the kinetic energy $(K / U)$ residual of the cuboid cavity at $R e=1050$, (b) Power spectral of the periodic data in figure 5.20. . . . 77

5.22 (a) Contour of the spanwise velocity $w(x, y, z)$ of the cuboid cavity at $R e=1050$, isosurface level are $w(x, y, z)= \pm 0.01$. (b) Grey isosurfaces are streamwise vorticity $\omega_{x}= \pm 0.15$, the blue isosurfaces are negative streamwise velocity $u(x, y, z) . \ldots \ldots \ldots \ldots \ldots$

5.23 Spectrum of open cavity cavity at $R e=1000 \ldots \ldots$. . . . . . . . 79 
5.24 Real part of the eigenfunction velocity field of the cuboid cavity at $R e=$ 1050 , Eigenfunctions normalized with $\max (\hat{u})$. From upper to lower are corresponding to the eigenmodes listed in table 5.9. Isosurfaces $\hat{u}= \pm 0.1$, $\hat{v}, \hat{w}= \pm 0.05$. (left): $\hat{u},($ middle) $: \hat{v}$ and (right): $\hat{w} . \ldots \ldots 80$

5.25 (a) Nonlinear simulation initialized using a single ST mode at initial amplitude $\epsilon=10^{-4}$ and $R e=1050$. (b) Upper: Temporal evolution of kinetic energy $K$. Lower: Power density spectrum of kinetic energy at $R e=1050$. . 81

5.26 The damping rates of the first three leading modes and the linear interpolation of ST mode. . . . . . . . . . . . . . . . . . . . . . 82

5.27 Comparison of the flow topology from the experiments and numerical simulations. (a) Instantaneous flow streamlines depicted from experiments by Crook et al. [31]. (b) Three-dimensional view of topological changes exert by ST mode at $R e=1050 . \ldots \ldots$. . . . . . . . . . . . . . .

5.28 (a) Streamlines of the base flow at $R e=1050$. (b) Streamlines of the flow reconstruction using the ST mode with lower amplitude $\epsilon=0.1$. (c) Streamlines of the flow reconstruction using CS mode (c) and CT mode (d) with lower amplitude $\epsilon=0.01 . \ldots \ldots$. . . . . . . . . . . . 84

5.29 (a)Normalized module of the ST mode, iso-surface value is 0.2 (grey) and 0.4 (blue). (b)Normalized module of the adjoint ST mode, iso-surfaces represent a value of 0.2 (grey) and 0.4 (blue). (c) Sensitivity function $\delta \lambda$ of the three-dimensional cavity at $R e=1050$. Iso-surfaces represent a normalized value of 0.3 (grey) and 0.7 (blue) . . . . . . . . . . 85

5.30 Contours of $\delta \lambda$ in $x-y$ center-plane of the cavity. . . . . . . . . . . 86

5.31 Time trace of streamwise velocity $u(3,0,1)$ and the details of the signal for the cuboid cavity at $R e=1100 \ldots \ldots \ldots$. . . . . . . . . 88

5.32 Power density spectrum of the streamwise velocity $u$ of probes (a) inside of the cavity $(3,-0.5,1.25)$ and (b) downstream of the cavity $(7,0.1,1.25)$. . 89

5.33 (a) Contour of the spanwise velocity $w(x, y, z)$ of the cuboid cavity at $R e=1100$, isosurface level are $w(x, y, z)= \pm 0.01$. (b) Grey isosurfaces are streamwise vorticity $\omega_{x}= \pm 1$, the blue isosurfaces are negative streamwise velocity $u(x, y, z)$.

5.34 (a) Contour of the average spanwise velocity $\bar{w}(x, y, z)$ at time domain [1200, 1600] of the cuboid cavity at $R e=1100$, isosurface level are $\bar{w}(x, y, z)=$ \pm 0.01 . (b-d) Visualization of the spanwise fluctuation $\Delta w(x, y, z, t)=$ $w(x, y, z, t)-\bar{w}(x, y, z)$ at three different times corresponding to approximately one-period of the three-dimensional instability. . . . . . . . . . .

5.35 Instantaneous visualization of the vortex generated by fluctuation velocity. The vertex identification is normalized by max value of $\mathrm{Q}$ criterion, isosurface of $\mathrm{Q}$ criterion is $0.007 \ldots \ldots \ldots$. . . . . . . . . . . 91

5.36 Surface streamlines of the three-dimensional open cavity flow at $R e=1100.91$

5.37 Anti-symmetric structure of three components (a) $u$, (b) $v$ and (c) $w$. The three components are normalized with $\max (u), \max (v)$ and $\max (w)$, respectively, iso-surface is $\pm 0.01 \ldots \ldots \ldots \ldots \ldots$

6.1 Quantitative evaluation of the structural sensitivity for three-dimensional cavity at $R e=1050 \ldots \ldots \ldots$. . . . . . . . . . . . . 94

6.2 Computational configuration of control Case 1. . . . . . . . . . . . 95 
6.3 Computational configuration of control Case 2. . . . . . . . . . . . 95

6.4 Computational grid of the Case $1 . \ldots \ldots \ldots$. . . . . . . . . 96 


\section{List of Tables}

1.1 The index of the previous studies of the open cavity in numerical simulation and experiment. 2.5D is the spanwise periodic cavity flow. . . . . . . 5 5

2.1 Classification of global linear theory appraches, where $\lambda$ is a complex value: $\lambda=\lambda_{r}+i \cdot \lambda_{i}, \lambda_{r}$ is damping/growth rate, $\lambda_{i}$ is the frequency. . . . . . 11

4.1 Parameters of the two-dimensional open cavity with aspect ratio $\mathrm{L} / \mathrm{D}=2$. . 28

4.2 Grid convergence study based on $u$ velocity for the two-dimensional open cavity at $\mathrm{Re}=1400$ using three meshes: M1 coarse, M2 medium and M3 fine. Grid convergence index obtained with $l=1.5$ and $n=1.25$. . . . . 28

4.3 The summary of the Boundary conditions of the open cavity simulation with OpenFOAM, Periodic ${ }^{\dagger}$ is special for the spanwise periodic open cavity case with the base flow from the spanwise extent of the 2D base flow. . . . 29

4.4 The eigenvalues of the least stable mode . . . . . . . . . . . . 30

4.5 Parameters of the open cavity. . . . . . . . . . . . . . . . . 31

4.6 Comparison of leading mode of 3D open cavity. . . . . . . . . . . . . 33

4.7 Comparison of the values of the minimum $u(0.5, y), v(x, 0.5)$ and maximum $v(x, 0.5)$ as well as the position with different resolutions at $R e=1000$ in the lid-driven cavity. $\dagger$ represents the spectral results from Albensoeder and Kuhlmann [2] . . . . . . . . . . . . . . . . . . . . . . . . 34

4.8 Comparison of the values of the minimum $u(0.5, y), v(x, 0.5)$ and maximum $v(x, 0.5)$ as well as the position with different resolutions at $R e=2000$ in the lid-driven cavity. . . . . . . . . . . . . . . . . 34

4.9 Damping rate and frequency of the leading stationary eigenmode and a traveling eigenmode obtained by solution of the global instability eigenvalue problem at $R e=2000 .^{\dagger}$ refers to the private communication with J.M. Pérez, ${ }^{*}$ is the results from Gómez et al. [53] . . . . . . . . . . . . 35

4.10 Mesh details of the 2D square open cavity. . . . . . . . . . . . . . . . 44

4.11 Unstable eigenvalues of the square open cavity at $R e=4140$ and $\beta=22 . \quad$. 45

4.12 The first three leading unstable eigenvalues of the spanwise periodic open cavity at the $R e=1000 \ldots \ldots$. . . . . . . . . . . . 49

4.13 Comparison results of the minimum and maximum velocities on the centerlines $v(x, 0.5,0.5)$ and $u(0.5, y, 0.5)$ of the cubic lid-driven cavity at $R e=$ 1000. $\mathrm{SCM}^{\dagger}$ spectral results from Albensoeder and Kuhlmann [2] and $\mathrm{FVM}^{*}$ finite volume method results from Gómez et al. [54], SEM represent current results from spectral element method. . . . . . . . . . . . . . . 52

4.14 Comparison of the three eigenvalues of the lid-driven cavity at $R e=1000$. $\quad 53$

5.1 Parameters characterizing the inflow Blasius boundary layer of the threedimensional open cavity with geometric ratio $L: W: D=6: 2: 1 \ldots \ldots \ldots$ 
5.2 The maximum absolute value of $|u|_{\max }$ at the location of $(x,-0.5,0.5)$ and $|w|_{\max }$ at the location of $(5,-0.5, z)$ inside cavity at $R e=1000 \ldots \ldots 58$

5.3 Comparison of the streamwise velocity $u(x, y)$ with three different height domain in the probe point $(x=L / 2, y=0, z=W / 2)$ of the cavity. . . . . 59

5.4 Mesh details of the cuboid open cavity. . . . . . . . . . . . . . 59

5.5 The first six leading eigenvalues of the cuboid cavity at $R e=900 . \quad \ldots$. . 63

5.6 The first six leading eigenvalues of the cuboid cavity at $R e=950 . \quad \ldots .68$

5.7 The first six leading eigenvalues of the cuboid cavity at $R e=1000 . \quad$. . . . 74

5.8 Comparison of the Strouhal number of the least eigenmode of two- and three-dimensional open cavity runs with the same length to depth ratio $L / D=6$ at $R e=1000$. The Rossiter prediction are calculated by (1.1). . . 76

5.9 The first six leading eigenvalues of the cuboid cavity at $R e=1050 \ldots$. . . 78

5.10 The damping rate and the corresponding $R e$ are used for the linear interpolation. . . . . . . . . . . . . . . . . . 82

5.11 Eigenvalue sensitivity to the size of the computational domain. . . . . . . 87

6.1 Details of the parameters of $\delta \lambda \ldots \ldots \ldots \ldots \ldots$. . . . . . . . . 93 


\section{Chapter 1}

\section{Introduction}

Viscous flows inside three-dimensional open cavities are of significant interest in fluid mechanics. This kind of flows have been comprehensively employed as engineering models for the understanding of different aeronautical devices, such as slotted flats or landinggear bays. Despite cavities have a simple geometry, the flow over this particular geometry has proven to be a challenging task due to a complex flow structure. The flow consists of rich flow features involving shear layers, centrifugal motions, shock waves, expansion waves, acoustic waves, and interactions amongst all these phenomena. All these physical features have been subject of an extensive research since the 1950's [72, 99, 104] and a large number of experimental and numerical studies of cavity flow have been carried out since then. Table 1.1 summarizes a list of these studies depending on their geometry and Reynolds number that will be detailed in what follows.

A large part of literature has addressed the flow structure and instability properties of two-dimensional and spanwise-periodic cavity flows. The shear layer instability, centrifugal instability and wake mode instability are well understood.

Shear layer instability, as the well known feature of low Mach number cavity flows, has been widely proved by experiment and numerical simulations in the cavity flow. Rossiter [100] was the first to describe the physical mechanism driving the self-sustained oscillations in cavity flows. He observed that, the small vortices generate at the leading edge by velocity shear, follow their convection and impinge on the trailing edge. This leads to mass injection inside of the cavity in an unsteady fashion, and the vorticity inside of the cavity interact with the flow over the cavity, finally strong fluctuations develop at the trailingedge corner. In the case of an incoming laminar boundary layer, these interactions are expected to be quasi-regular. Hence the physical mechanisms of self-sustained oscillations can be understood as a feedback process. The schematic diagram of self-sustained fluid mechanism is presented in figure 1.1, a fluid with a far-field velocity $U$ following streamwise direction $x$ flows over a length to depth ratio L/D cavity.

Based on this experimental observations, Rossiter [100] developed his semi-empirical formula to predict the resonance frequencies.

$$
S t_{L}=\frac{f L}{U}=\frac{n-\alpha}{M+1 / \kappa},
$$

where $n=1,2,3, \ldots$ leads to the frequency of the Rossiter mode I, II, III... and $M$ is the freestream Mach number. The empirical constant $\alpha$ represents the average convection speed of vortices traveling over the cavity normalized by freestream speed. $\kappa$ is the phase delay of vortices adainst the upstream traveling acoustic waves. In the original experiment from Rossiter, $\alpha=0.25$ and $\kappa=0.57$.

Besides the shear layer instability, centrifugal instability represents one type of lowfrequency oscillation associates to the main recirculation eddy inside cavity. Brès and Colonius [19] conducted a linear instability analysis of two-dimensional steady stable flow 


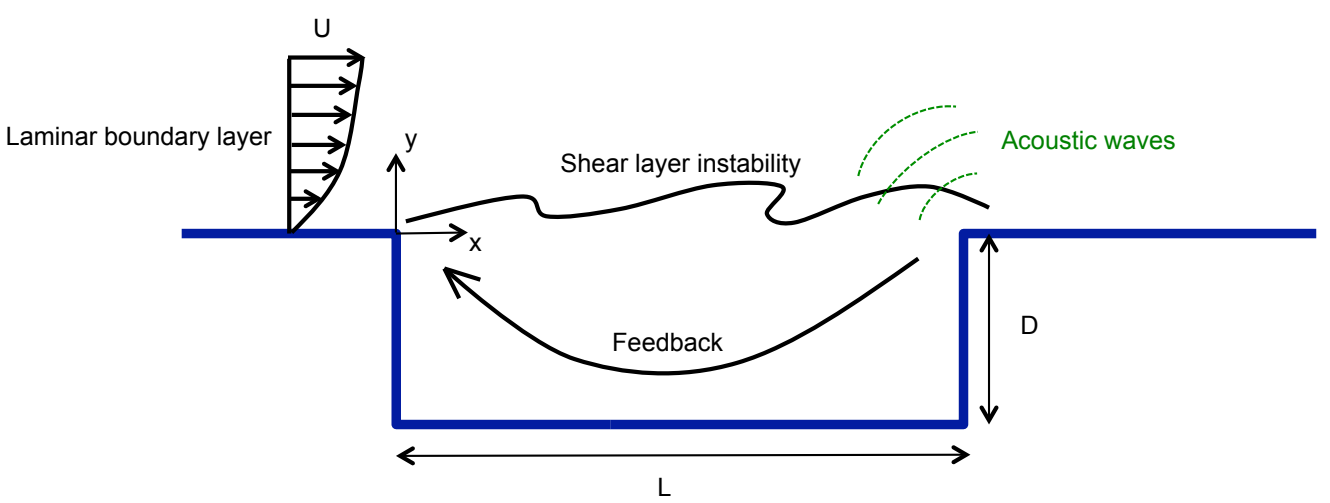

Figure 1.1: Sketches of global instability analysis examples

for a periodic cavity in the spanwise direction, and were first to report that this centrifugal instability is responsible for the loss of stability of two-dimensional cavity flows to three-dimensional instabilities. In their numerical experiments of spanwise-periodic cavities, they observed that the spanwise oscillations had a frequency one order of magnitude lower than the two-dimensional Rossiter mode. Also, they noted that the spanwise oscillations had a wavelength of one cavity depth. This observation was in agreement with the experimental visualizations by Faure et al. [39].

Later, Faure et al. [40] experimentally confirmed that the three-dimensional flow structures in the open cavity were independent of the shear-layer instability. Recently, de Vicente et al. [34] performed an extensive investigation of the onset of the three-dimensional centrifugal instabilities in a $L / D=2$ spanwise-periodic incompressible cavity flow and compared their results to experiments, reaching an qualitative agreement. In the same line, Meseguer-Garrido et al. [83] presented an extension of the theoretical results of de Vicente et al. [34] for a wide range of parameters. They obtained empirical relations for the Strouhal numbers of the possible centrifugal modes. Recent numerical results on spanwiseperiodic open cavities provided by Citro et al. [26] are also in agreement with previous investigations.

A new understanding of cavity flow arises as cavity aspect ratio increases. A different behavior of the shear, termed as wake mode, was firstly revealed by Gharib and Roshko [48] firstly. In their experiments of incompressible flow over an axisymmetric cavity, they observed that under certain Reynolds numbers or length to depth ratio L/D, the cavity flow can behave as a bluff body flow. As such, the flow features changed substantially; the mean streamlines were no longer horizontal along the cavity and the primary recirculation eddy inside the cavity was lost. Hence, they stated that the wake mode was intrinsically different to the shear layer mode, and it is always related to the unstable fashion. Pereira and Sousa [89] and Colonius et al. [30] observed similar behaviors in two-dimensional direct numerical simulation of the cavity flow. Later, Rowley et al. [101] investigated the onset of the shear layer mode and the wake mode in the open cavity flow using modal linear theory. The study clearly demonstrated that the wake mode was a two-dimensional feature and it occurred in large length to depth ratio cavities.

In this context, it is worth mentioning that Komerath et al. [71] established a classification of open cavities based on their length to depth ratio and their behavior. They stated that the flow over cavity can be classified into three types: (1) open flow :L/D $\leq 10,(2)$ 
closed flow: $L / D>13$ and (3) transitional flow: $10<L / D \leq 13$. The recent work of Sun et al. [110] on compressible flow over cavities with aspect ratio aspect ratio $L / D=2$ and $L / D=6$ also corroborates these different possible behaviors.

Although these well-known instabilities in the cavity flow have been validated in many studies, apparent discrepancies have been found with respect to three-dimensional wallbounded open cavity flows. For instance, Sarohia [103] investigated the laminar axissymmetric flow over shallow cavities and found that a large lateral motion of shear layer occurs in the proximity of the downstream lip. This motion leads to a periodic shedding of vortices at a frequency of cavity oscillation different to the Rossiter frequencies.

Forestier et al. [46] studied a deep cuboid cavity with an aspect ratio $L / D=0.42$ and depth to width $D / W=1$ at Reynolds number $R e_{L} \approx 8.6 \times 10^{5}$ based on the cavity length. They observe that the flow was subject to regular self-sustained oscillations with a highly two-dimensional and periodic organization of the mixing layer over the cavity. They pointed out the formation of coherent vortices close to the boundary layer separation had similarity to the 'collective interaction mechanism' introduced by Ho and Huang [60]. Larchevêque et al. [73] identified two different regions in the shear-layer by means of large-eddy simulation of a $L: D: W=5: 1: 1$ cavity at $R e_{L}=7 \times 10^{6}$. The first region of the shear was dominated by two-dimensional Rossiter modes and the subsequent second region behaved very similarly to a free mixing layer. Results from Forestier et al. [45] and Larchevêque et al. [74] in a similar open cavity $L: D: W=2: 1: 0.42$ at $\operatorname{Re}_{D}=8.6 \times 10^{5}$ revealed that the mean flow could present asymmetry inside the cavities.

The observation which expresses the asymmetry structure existed in the three-dimensional cavity has also be confirmed subsequently. Crook et al. [32] investigated the flow topology of the time-averaged flow of a cavity with aspect ratio $L: D: W=6: 2: 1$ at $R e=4.3 \times 10^{4}-$ $3.4 \times 10^{5}$. Apart from the previous observed flow features inside the cavity, they pointed out the existence of a single vortex located near the cavity centerline. This support that this vortex was responsible for asymmetries in the cavity and postulated that the observed asymmetry in the time-average flow field was due to the asymmetric in the instantaneous flow field, which switched between two extremes at low frequency.

Zhang and Naguib $[125,126]$ corroborated these observations by performing experiments of a cuboid shallow cavity flow with fixed $L / D=3.4$ and various values of $W / D$. They observed that low frequency fluctuations were associated to the centrifugal instability identified by Brès and Colonius [19]. In this context, Yao et al. [123] investigated the unsteady incompressible flow past three-dimensional rectangular cavities at different aspect ratios. They observed that as the Reynolds number increases, both three-dimensional centrifugal modes and two-dimensional shear layer modes coexist.

Recently, the effect of the sidewalls in the cavity flow has been studied by George et al. [47]. The experiment were carried on $M a_{\infty}=1.4$, over full and finite span rectangular cavities with aspect ratio $L: D=6: 2$. The unsteady surface pressure and particle image velocimetry results revealed the dominant mode switches from the third to the second Rossiter mode when comparing the full span to the finite span. Also results on flow control of the cavity by leading edge slot blowing technique were presented. The research performed on subsonic cavity flow is presented in the work Zhang et al. [128], who analyzed three-dimensional cavity flow via steady blowing fixed upstream lip. Additionally, they expected that the global instability analysis should be consider as the further researches, which may be provide insight as to the optimal properties of the disturbance.

The previous discuss show that three-dimensional wall-bounded flow gives rises to 
rather complex flow structure patterns, finding instability properties and indicating responsibility of flow unsteadiness are still an open topic nowadays. In this context, an attempt to understand the instability onset of open cavity flows bounded by lateral walls is made in this thesis.

Direct numerical simulation of an open cavity bounded by lateral walls will be performed in order to investigate the instability onset and prove whether the spanwiseperiodicity assumption is adequate to accurately describe the flow features of a realistic cavity. Additionally, we will apply global instability analysis Theofilis [112] to identify the linear modes responsible for the instability. To ease comparison with the flow features observed in the literature, we will choose the same cavity geometry as Crook et al. [32].

Moreover, a novel insight into the theoretical flow control are performed. The cavity control follows a frame of receptivity and structural sensitivity analysis. 


\begin{tabular}{|c|c|c|c|c|c|c|}
\hline References & Type & $M_{\infty}$ & $L / D$ & $L / W$ & $R e_{D}$ & $\theta_{0}$ \\
\hline Sarohia [104] & $2 \mathrm{D}$ & 0.015 & $0-50$ & - & $2 \times 10^{4}-10^{5}$ & - \\
\hline Gharib and Roshko [48] & $2 \mathrm{D}$ & 0.00015 & $\begin{array}{c}0.6780 .985 \\
1.081\end{array}$ & - & $1.2 \times 10^{4}$ & 0.041 \\
\hline Rowley et al. [101] & $2 \mathrm{D}$ & $0.2-0.8$ & $\begin{array}{c}1234 \\
568\end{array}$ & - & $750-3748.3$ & $0.0162-0.0985$ \\
\hline$[122]$ & $2 \mathrm{D}$ & $0.04-0.9$ & 12 & - & 7500 & $0.0043-0.0292$ \\
\hline Sun et al. [110] & $2 \mathrm{D}$ & $\begin{array}{l}0.1-1.6 \\
0.1-1.2\end{array}$ & $\begin{array}{l}2 \\
6\end{array}$ & - & $\begin{array}{l}238-3802 \\
132-1214\end{array}$ & 0.0379 \\
\hline Özsoy et al. [84] & $2.5 \mathrm{D}$ & $0-0.0582$ & 4 & 3.75 & $\begin{array}{c}4000 \\
9000 \\
13000\end{array}$ & $\begin{array}{l}0.035 \\
0.028 \\
0.025\end{array}$ \\
\hline Faure et al. [39] & $2.5 \mathrm{D}$ & $0.1-1.6$ & 2 & 0.333 & $275-5335$ & - \\
\hline Brès and Colonius [19] & $2.5 \mathrm{D}$ & $0.1-0.8$ & 124 & $\begin{array}{cl}0.2 & 0.25 \\
2 & 3.2\end{array}$ & $755-6960$ & $0.0033-0.0571$ \\
\hline Faure et al. [40] & $2.5 \mathrm{D}$ & 0.01 & $0.25-2.5$ & $0.125-4.8$ & $1150-36800$ & - \\
\hline de Vicente et al. [34] & $2.5 \mathrm{D}$ & - & 2 & $0.796-6.369$ & $1100-2400$ & 0.0363-0.0392 \\
\hline Meseguer-Garrido et al. [83] & $2.5 \mathrm{D}$ & - & $\begin{array}{c}1.0,1.1,1.2 \\
1.31 .4 \\
1.523\end{array}$ & $0.0455-\infty$ & $800-4600$ & $0.1157-0.3$ \\
\hline Citro et al. [26] & $2.5 \mathrm{D}$ & - & 1 & $\begin{array}{l}2.134 \\
3.503\end{array}$ & $\begin{array}{l}1370 \\
4140\end{array}$ & $\begin{array}{l}0.0113 \\
0.0065\end{array}$ \\
\hline Forestier et al. [46] & $3 \mathrm{D}$ & 0.8 & 0.42 & 0.42 & $2.048 \times 10^{6}$ & 0.0054 \\
\hline Yao et al. [124] & $3 \mathrm{D}$ & - & $\begin{array}{l}1 \\
2 \\
4\end{array}$ & 0.333 & $\begin{array}{c}300010000 \\
3000 \\
300010000\end{array}$ & $\begin{array}{c}0.0080 .015 \\
0.0130 .028 \\
0.013 \\
0.013\end{array}$ \\
\hline Larchevêque et al. [73] & $3 \mathrm{D}$ & 0.85 & 5 & 5 & $1.4 \times 10^{6}$ & - \\
\hline Ashcroft and Zhang [5] & $3 \mathrm{D}$ & $\sim 0.1$ & $\begin{array}{l}2.0 \\
3.0 \\
4.0\end{array}$ & $\begin{array}{l}0.111 \\
0.167 \\
0.222\end{array}$ & $\begin{array}{l}1.1 \times 10^{5} \\
1.3 \times 10^{5} \\
1.4 \times 10^{5}\end{array}$ & - \\
\hline $\begin{array}{l}\text { Larchevêque et al. [74] } \\
\text { Zhang and Naguib }[125,126]\end{array}$ & $3 \mathrm{D}$ & $\begin{array}{c}0.8 \\
-\end{array}$ & 3.4 & $\begin{array}{cl} & 0.42 \\
0 & 0.479 \\
1.417 & 0.971\end{array}$ & $\begin{array}{c}4.3 \times 10^{5} \\
4067 \\
12200\end{array}$ & $\begin{array}{c}- \\
0.169 \\
0.252\end{array}$ \\
\hline Crook et al. [32] & $3 \mathrm{D}$ & - & 6 & 3 & $\begin{array}{l}7.167 \times 10^{3} \\
5.667 \times 10^{4}\end{array}$ & $\begin{array}{c}0.01 \\
0.011\end{array}$ \\
\hline Zhang et al. [128] & $3 \mathrm{D}$ & $\begin{array}{c}0.330 .430 .53 \\
0.630 .73\end{array}$ & 6 & 1.56 & $4 \times 10^{5}$ & $\begin{array}{l}4.46 \times 10^{-4} \\
5.09 \times 10^{-4}\end{array}$ \\
\hline George et al. [47] & $3 \mathrm{D}$ & 1.4 & 6 & 3 & $4 \times 10^{6}$ & $3.187 \times 10^{-4}$ \\
\hline
\end{tabular}

Table 1.1: The index of the previous studies of the open cavity in numerical simulation and experiment. $2.5 \mathrm{D}$ is the spanwise periodic cavity flow. 


\subsection{Novelty and Overview}

In the past, cavity flow has been analyzed over different configuration ratios from twodimensional to two and half-dimensional (spanwise periodic flow) and three-dimensional, either experiments or numerical simulations. The majority of this research focuses on the high Mach and high Reynold number flows. This leaves the onset of the first instability from laminar transits unsteady state still an open question in the cavity flow. Our study is in an attempt to fill the gaps of low Reynolds number three-dimensional cavity flow in the literature. For the first time, linear instability is applied to three-dimensional cavity flow.

This thesis includes direct numerical simulation (DNS) of an three-dimensional wallbounded open cavity, the discussion of the first instability of this particular flow and the instability properties comparison with the spanwise-periodic counterpart. Insight into the receptivity and structure sensitivity are obtained in order to control the flow instability. To ease comparison with the flow features observed in the literature, we will choose the same cavity geometry as Crook et al. [32].

In this context, achievements have been obtained in OpenFOAM by implementing direct and adjoint linearized Navier-Stokes solver. A time-stepping method, iterative approach of solving eigenvalue problem, coupled with the previous modified code has been used to investigate three-dimensional problem. The code modification and the successful numerical simulations are described in Chapter 3 and Chapter 4, respectively. Owing to two main reasons, three-dimensional open cavity flow with lateral walls is investigated using Nek5000 rather than OpenFOAM. Firstly, second order finite volume numerical scheme in OpenFoam is less sufficient for researching small amplitude perturbation problem. Secondary, the requirement of huge computational resource fetters investigation for three-dimensional complex flow.

The present thesis is divided into different chapters, each one addressing a different objective of the present work:

- Chapter 2 details the instability theory. Different instability approaches depend on the dimensionality of the base flow. In this context, the attention is focused on the BiGlobal and TriGlobal analyses.

- Chapter 3 describes the different numerical methods used in this thesis, from the standard second-order Finite Volume Method (OpenFoam) to high-order spectral element method (Nek5000). All of these numerical methods follow a time-stepping method.

- Chapter 4 presents the implementation and validation of the numerical methods used in this thesis. Two parts of the work are presented. In the first part, the validations are carried out from $2 \mathrm{D}$ to $2.5 \mathrm{D}$ cavity flows in OpenFoam. The second part of work is performed with Nek5000, and it includes the two-dimensional cylinder flow, spanwise periodic cavity flow, and three-dimensional lid-driven cavity cases.

- Chapter 5 documents the global instability analysis of three-dimensional long open cavity with lateral walls. The geometry and flow conditions are briefly discussed. A different flow structures are found as the flow transits from stable to saturated unstable states. A new three-dimensional shear layer mode is observed, with a more 
complex flow structure than the spanwise periodic counterpart. Additionally, the structural sensitivity region has been detected using the adjoint-based theory .

- Chapter 6 proposes a method to examine where a spatial modification of the base flow has the largest impact on the instability features. The spatial variation of base flow is based on the structural sensitivity result of three-dimensional open cavity in the previous chapter 5 . It has relevant implications for the passive control of instabilities.

- Chapter 7 summarizes the final conclusions and suggests further lines of research.

\subsection{Publications}

Most of the material in this thesis, along with additional specific details, has been presented in the following publications:

- Journal Papers (JCR Peer-Reviewed)

- Q. Liu, F. Gómez, J. M. Pérez and V. Theofilis. "Instability and sensitivity analysis of flows using OpenFOAM". Accepted in Chinese Journal of Aeronautics.

- Q. Liu, F. Gómez and V. Theofilis. "TriGlobal linear instability analysis of incompressible flow over a cuboid cavity". Journal of Fluid Mechanics, (In preparation).

- Book Chapters (Peer-Reviewed with ISBN/ISSN)

- Q. Liu, F. Gómez and V. Theofilis. Flow instabilities in an open aircraft bay model cavity. AIAA Aviation and Aeronautics Forum and Exposition (AIAA AVIATION 2016). (Under revision).

- Q. Liu, F. Gómez, and V. Theofilis. Linear instability analysis of incompressible flow over a cuboid cavity. Procedia IUTAM, Volume 14, 2015, pp 511-518.

- Q. Liu, A. Kazakidi, M.A. Medeiros and V. Theofilis. Numerical Simulation of a Synthetic Jet with OpenFOAM. Fluid Mechanics and Its Applications, Volume 107, 2015, pp 197-202, Springer International Publishing.

- H. Zhang, J. Li and Q. Liu. Flight loads analysis of a maneuvering transport aircraft. Advance Materials Research, Vol. 1016, pp. 460-464.

- F. Gómez, V. Theofilis, P. Paredes, Q. Liu and W. He. On the role of global flow instability analysis in closed loop flow control. 2012, AIAA paper 2012-2679.

- List of talks

- 2015 Sept. 28-Oct. 4 Global flow instability and control symposium VI, Crete, Greece.

- 2013 Sept. 4-6. "Numerical simulation of a synthetic jet with OpenFoam", Instability and control of Massively Separated flows, Monash Center in Prato, Italy.

- 2012 Nov. 29-30. 2nd Annual Review Meeting of ICOMASEF consortium, Universidade de São Paulo, Brazil. 


\section{Chapter 2}

\section{Hydrodynamic Instability}

Hydrodynamic instabilities play an essential role in the investigation of the sequence of physical mechanisms leading laminar flow through transition to turbulence. Although turbulent is encountered in nature and engineering application in the majority of fluid, it is typically unwelcome in aerodynamic application, and directly solving the turbulence flow is still a huge challenge. Hydrodynamic instability analysis is of great importance in many flows of engineering relevance, it puts forward a theoretic basis for flow control. As far as we know, flow control plays an important role to enhance the performance of the aerodynamic devices, suppresses the flow separation, delays the evolution to transition and turbulence, depresses acoustic noise or decreases the oscillation which could induce the structure fatigue.

Instability analysis theory has occupied a substantial part of fluid mechanics research for over a century. The bulk of numerical efforts has been confined into analyzing the decay/growth of a small amplitude perturbation superimposed upon a steady or unsteady laminar base flow. The growth amplitude of the perturbation leads to a bifurcation from a steady laminar state to unsteady state. The assumption of asymptotic instability leads to a generalized large-scale eigenvalue problem. The spectrum of the linear global modes are related to the numerical solution of this large matrix, of which eigenvectors are known as global modes, and the corresponding eigenvalues represent the frequencies and growth/damping ratios of the small perturbation.

With flow instability dominating the unsteady motion existing at even low Reynolds numbers, it is expected that classification of the flow manifestations provides intuitively understanding of properties of flow. The stability of a flow can be determined by calculating the response of an impulse at the original of time and space, say $x=0$ and $t=0$. If the amplitude of the response dies out in all space, the flow is stable. If it amplifies, a further distinction is necessary.

If the response is convected away from $x=0$, the flow is locally convective unstable, as see in figure 2.1(a). Boundary layer flow is an example of convective unstable flow, small amplitude perturbations develop inside a thin boundary layer over a fairly long distance, the flow goes through laminar, transition until turbulence, forms TS waves, generates spanwise vorticity, and finally, three-dimensional vortices break down.

If the response expands around $x=0$, the flow is locally absolute unstable, as seen in figure 2.1(b). Instability appearing around a cylinder flow belongs to this kind of unstable flow.

\subsection{Navier-Stokes equations}

The incompressible continuity equation and Navier-Stokes equations are considered,

$$
\nabla \cdot \mathbf{u}=0
$$




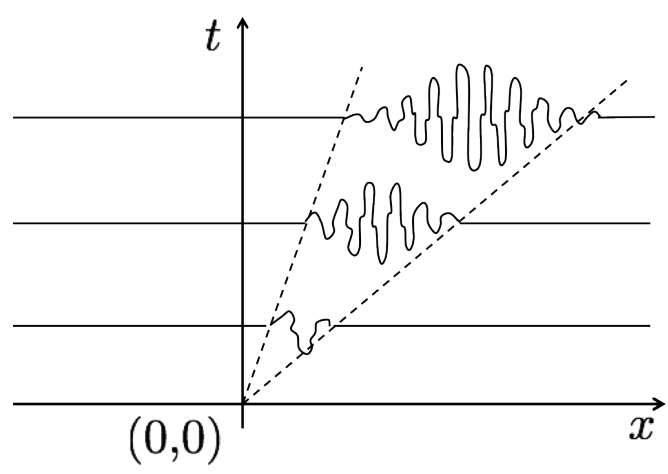

(a) Convective unstable

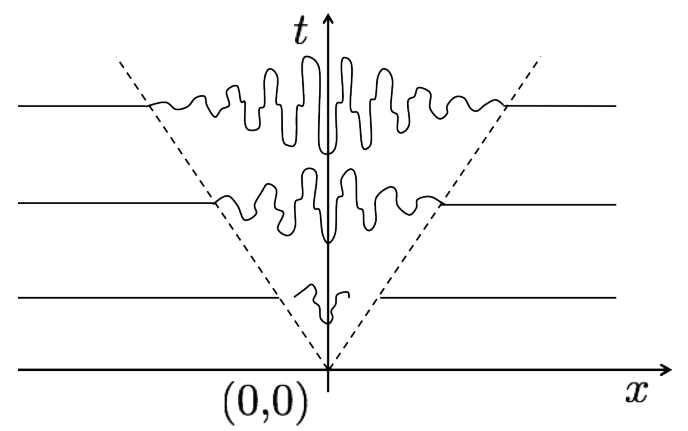

(b) Absolute unstable

Figure 2.1: Sketches of typical impulse responses

$$
\frac{\partial \mathbf{u}}{\partial t}+\mathbf{u} \cdot \nabla \mathbf{u}=-\nabla p+R e^{-1} \nabla^{2} \mathbf{u}
$$

$\mathbf{u}=(u, v, w)$ is the velocity in three directions, streamwise $x$, wall normal $y$ and spanwise direction $z$, respectively. $p$ is the pressure. Re is the Reynolds number,

$$
R e=U L / \nu,
$$

where $U$ and $L$ are the characteristic velocity and length scales, respectively. $\nu$ is the kinematic viscosity.

\section{$2.2 \quad$ Instability analysis theory}

Flow instability theory deals with the evolution of a small-amplitude perturbation in time and space, superimposed upon a steady or unsteady laminar base flow. The flow defined by the governing equations (2.1) is decomposed as a base flow $\overline{\mathbf{u}}$ and unsteady smallamplitude perturbations $\tilde{\mathbf{u}}$,

$$
\mathbf{u}=\overline{\mathbf{u}}(\mathbf{x})+\epsilon \tilde{\mathbf{u}}(\mathbf{x}, t)
$$

where $\epsilon<<1, \mathbf{x}$ is the spatial coordinations depending on the dimensionality of the flow.

By introducing the decomposition (2.3) in to the governing equations (2.1) and retaining the infinitesimal terms $O(\epsilon)$, the linearized Navier-Stokes equations (LNSE) are obtained

$$
\begin{aligned}
\nabla \cdot \tilde{\mathbf{u}} & =0 \\
\frac{\partial \tilde{\mathbf{u}}}{\partial t}+\overline{\mathbf{u}} \cdot \nabla \tilde{\mathbf{u}}+\tilde{\mathbf{u}} \cdot \nabla \overline{\mathbf{u}} & =-\nabla p+R e^{-1} \nabla^{2} \tilde{\mathbf{u}} .
\end{aligned}
$$

Writing (2.4b) in matrix form, the initial-value-problem (IVP) is

$$
\frac{\partial \tilde{\mathbf{u}}}{\partial t}=\mathbf{A} \tilde{\mathbf{u}},
$$

where $\mathbf{A}$ is the Jacobian matrix of the right-hand side of the Navier-Stokes equation. Specific comments on the dependence of these quantities on the spatial coordinates $\mathbf{x}$ 
2.2. Instability analysis theory

\begin{tabular}{l|l|lll}
\hline & Denomination & \multicolumn{2}{|c}{ Basic State Assumptions } & Phase Function $\Theta$ \\
\hline \multirow{3}{*}{ Global } & TriGlobal & - & $\overline{\mathbf{u}}(x, y, z)$ & $\exp (\lambda \cdot t)$ \\
& PSE-3D & $\partial_{x} \overline{\mathbf{u}}<<\partial_{y} \overline{\mathbf{u}}, \partial_{z} \overline{\mathbf{u}}$ & $\overline{\mathbf{u}}\left(x^{*}, y, z\right)$ & $\exp \left(\int \alpha\left(x^{*}\right) d x^{*}+\lambda \cdot t\right)$ \\
& BiGlobal & $\partial_{z} \overline{\mathbf{u}}=0$ & $\overline{\mathbf{u}}(x, y)$ & $\exp (\beta z+\lambda \cdot t)$ \\
\hline Non-Local & PSE & $\partial_{x} \overline{\mathbf{u}}<<\partial_{y} \overline{\mathbf{u}} ; \partial_{z} \overline{\mathbf{u}}=0$ & $\overline{\mathbf{u}}\left(x^{*}, y\right)$ & $\exp \left(\int \alpha\left(x^{*}\right) d x^{*}+\beta z+\lambda \cdot t\right)$ \\
Local & OSE & $\partial_{x} \overline{\mathbf{u}}=\partial_{z} \overline{\mathbf{u}}=0$ & $\overline{\mathbf{u}}(y)$ & $\exp (\alpha x+\beta z+\lambda \cdot t)$ \\
\hline
\end{tabular}

Table 2.1: Classification of global linear theory appraches, where $\lambda$ is a complex value: $\lambda=$ $\lambda_{r}+i \cdot \lambda_{i}, \lambda_{r}$ is damping/growth rate, $\lambda_{i}$ is the frequency.

and time $t$, will be made in the following. The operator $\mathbf{A}$ is associated with the spatial discretization of the linearized Navier Stokes equations (LNSE) and comprise the base state $\overline{\mathbf{u}}$. The separation between time and space coordinates in (2.5) permits introducing a Fourier decomposition in time. The perturbation term is usually written as the product of an amplitude function $\hat{\mathbf{u}}$ with a phase function $\Theta, \tilde{\mathbf{u}}=\hat{\mathbf{u}} \Theta$. Table 2.1 summarizes the different instability approaches associated on the dimensionality of the base flow analyzed.

At the strongest level of approximation local instability analysis, the base flow is considered homogeneous along two spatial directions and then the parallel flow approximation is made. In this approximation, the streamwise and spanwise derivatives of the mean flow are assumed to be negligible. One can then assume small-amplitude perturbations $\tilde{\mathbf{u}}$ in (2.3) as following expression,

$$
\tilde{\mathbf{u}}(x, y, z, t)=\hat{\mathbf{u}}(y) e^{\mathrm{i} \cdot(\alpha x+\beta z)+\lambda t}+\text { c.c. }
$$

with complex wave numbers $\alpha, \beta$ and complex frequency $\lambda$. The periodicity lengths $L_{x}=2 \pi / \alpha$ and $L_{z}=2 \pi / \beta$ have been imposed to the disturbances shape in the $x-$ and $z$ - direction respectively.

Substituting (2.6) in (2.4), Once the appropriate boundary condition imposed, these stability equations turn into an eigenvalue problem whereby eigenfunctions exist only is $\alpha, \beta$ and $\lambda$ satisfy a dispersion relation [3, 28,62]. For simplicity, the wavenumber $\beta$ in (2.6) is taken as a real parameter like a control parameter $R e$, without losing any of the essential characteristics of instability.

$$
D[\alpha, \lambda ; \beta, R e]=0 .
$$

The dispersion relation $D[\alpha, \lambda ; \beta, R e]$ is equivalent in space differential operator $D[\mathrm{i} \partial / \partial x,-\mathrm{i} \partial / \partial t ; \mathrm{i} \partial / \partial z, R e]$ in physical space[11, 12, 20,61, 76, 109], the eigenfuction satisfies

$$
D\left(\mathrm{i} \frac{\partial}{\partial x}, \mathrm{i} \frac{\partial}{\partial t} ; \frac{\partial}{\partial z}, R e\right) \hat{\mathbf{u}}=\mathbf{0} .
$$

The dispersion relation (2.8) maps points between the complex $\alpha$-plane and the complex $\lambda$-plane. The disturbances characterized by $\alpha$ and $\lambda$ can grow/decay in space (spatial local instability), in time (temporal local instability) separately, or simultaneously grow/decay in space and decay/growth temporally (spatio-temporal instability)[62, 68].

In temporal local stability analysis, the quantity $\alpha$ in (2.6) is real wavenumber parameters, the sought complex eigenvalue is $\lambda=\lambda_{r}+\mathrm{i} \cdot \lambda_{i}$, where $\lambda_{r}$ is the growth/damping rate and $\lambda_{i}$ is the circular frequency. In spatial local stability analysis, $\lambda$ is a real frequency parameter and $\alpha=\alpha_{r}+\mathrm{i} \alpha_{i}$ is complex eigenvalue, $\alpha_{r}$ is related with the periodicity length 
along the homogeneous spatial direction, through and $\alpha_{i}$ is the spatial amplification/damping rate. In spatio-temporal stability analysis, both $\alpha$ and $\lambda$ can be complex. The velocity of wave crests $c=\lambda / \alpha$ is called phase velocity and the velocity of the envelope of the wave crests $c_{g}=\partial \lambda / \partial \alpha$ is group velocity. Absolutely instability would appear if the wave has $c_{g}=0$ and the temporal growth rate $\lambda_{r}>0$. The border of absolute instability and convective instability can be defined by the parametric region of those wave-like disturbances.

The traditional instability theory are based on the quasi-parallel flow assumption. It does not account for such shear layer flow, boundary-layers, jet and far wakes, which base flow has a weak variation in one direction (e.g. x direction) and strong variation in another direction (e.g. y direction). PSE have made a successful effort in this kind of flow. The base flow evolves in the streamwise direction with a large scale $X$, while the disturbances evolves with a small scale $x$. The small-amplitude perturbations $\tilde{\mathbf{u}}$ in (2.3) can be decomposed as the following expression,

$$
\tilde{\mathbf{u}}(x, y, z, t)=\hat{\mathbf{u}}(X, y) e^{\mathrm{i}\left(\int_{X} \alpha(X) d X+\beta z\right)+\lambda t}+\text { c.c. },
$$

where amplitude function $\hat{\mathbf{u}}$ and the streamwise wavenumber $\alpha$ are slowly varying functions on $X$. The PSE are valid for convective unstable flows. See more details in Bertolotti [13], Herbert [57].

\subsubsection{BiGlobal stability analysis}

Assuming that the base flow varies in two out of the three spatial directions. For instance, lid-driven cavity with a large span in $z$ direction in figure 2.2(a), the base flow is dependent on the $x-y$ plane, whereas the perturbation is three-dimensional, with the homogeneous $z$ direction, the periodicity length is assumed $L_{z}=2 \pi / \beta$. The small-amplitude perturbations $\tilde{\mathbf{u}}$ in (2.3) can be decomposed as

$$
\tilde{\mathbf{u}}(x, y, z, t)=\hat{\mathbf{u}}(x, y) e^{(\mathrm{i} \cdot \beta z+\lambda t)}+\text { c.c.. }
$$

For the temporal BiGlobal instability case, $\beta$ is a real number and sought complex eigenvalue is $\lambda=\lambda_{r}+\mathrm{i} \cdot \lambda_{i}$. For the spatial BiGlobal instability case, $\beta$ is the complex number, and $\lambda$ is the real number[112].

\subsubsection{TriGlobal stability analysis}

TriGlobal stability analysis is the most general case with the base flow and perturbations being non-homogeneous in the three coordinates. The small-amplitude perturbations $\tilde{\mathbf{u}}$ in (2.3) can be decomposed

$$
\tilde{\mathbf{u}}(x, y, z, t)=\hat{\mathbf{u}}(x, y, z) e^{\lambda t}+\text { c.c. },
$$

where $\lambda=\lambda_{r}+\mathrm{i} \cdot \lambda_{i}, \lambda_{r}$ is the growth rate and $\lambda_{i}$ is the circular frequency. As shown in figure $2.2(\mathrm{c})$, three-dimensional wall-bounded open cavity flow has three non-homogeneous directions, it is a TriGlobal stability analysis example.

Substituting (2.5) by TriGlobal decomposition (2.11), it leads to the generalized matrix eigenvalue problem (EVP),

$$
\lambda \hat{\mathbf{u}}=\mathbf{A} \hat{\mathbf{u}} .
$$




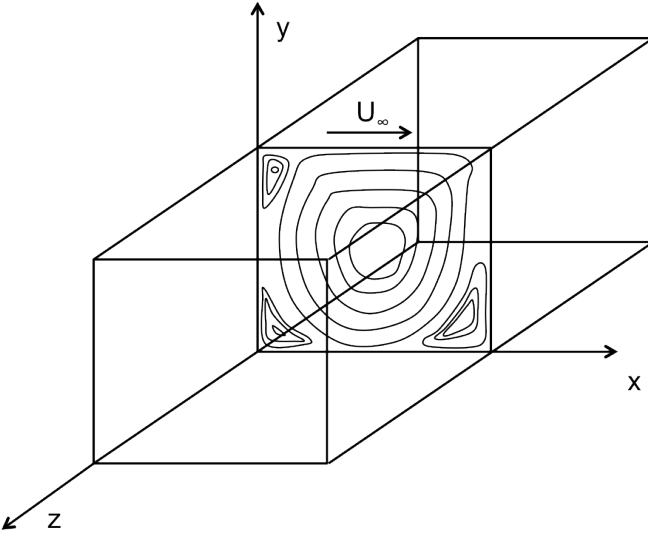

(a) BiGlobal example

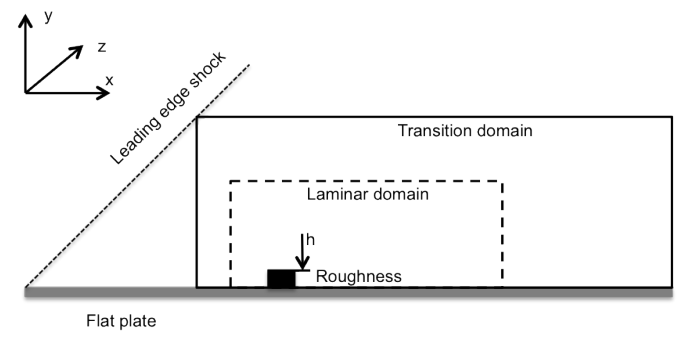

(b) PSE-3D example

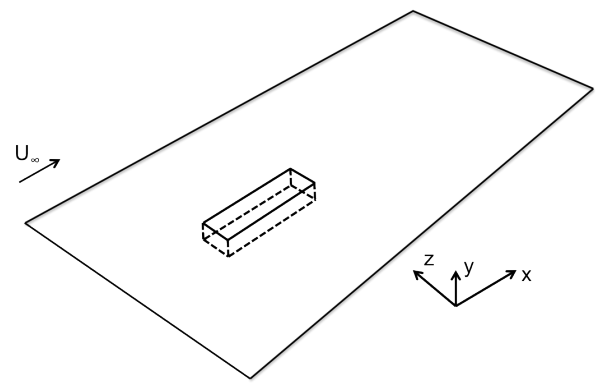

(c) TriGlobal example

Figure 2.2: Sketches of global instability analysis examples

\subsection{Determination of the Instability Core: Structure Sensi- tivity}

The adjoint-based sensitivity analysis has been widely applied from optimal shape design $[66,90]$ to model-based optimal control of fluid flows [70]. The adjoint analysis yields a very efficient way to determine the receptivity properties of the flow, which reveals the initial disturbance amplitude and the influence of external excitation on this flow. Thus, inspecting the adjoint eigenfunctions and assessing the receptivity regions of the flow provide the precious information to the structural sensitivity. An overview of recent developments in adjoint methods studying hydrodynamic stability has been compiled by Luchini and Bottaro [79].

The structure sensitivity of the eigenmodes to modifications of the base flow can be calculated with the construction of a bi-orthogonal or adjoint operator $\mathbf{A}^{*}[50,58,59,79$, 80], with adjoint velocity and pressure field $\hat{\mathbf{v}}$ and $m$. These adjoint equations can be written in a similar fashion (2.4), adjoint linearized Navier-Stokes equations are

$$
\begin{aligned}
\nabla \cdot \tilde{\mathbf{v}} & =0 \\
-\frac{\partial \tilde{\mathbf{v}}}{\partial t}+\overline{\mathbf{u}} \cdot \nabla \tilde{\mathbf{v}}+\tilde{\mathbf{v}} \cdot \nabla \overline{\mathbf{u}} & =-\nabla m+R e^{-1} \nabla^{2} \tilde{\mathbf{v}},
\end{aligned}
$$

The adjoint TriGlobal ansatz is introduced,

$$
\tilde{\mathbf{v}}(x, y, z, t)=\hat{\mathbf{v}}(x, y, z) e^{-\lambda t}+\text { c.c.. }
$$


Combination of the ansatz (2.14) with (2.13b) leads to the adjoint eigenvalue problem,

$$
-\lambda \hat{\mathbf{v}}=\mathbf{A}^{*} \hat{\mathbf{v}}
$$

or equivalently,

$$
\hat{\mathbf{v}}(\mathbf{A}-\lambda \mathbf{I})=\mathbf{0} .
$$

In the spatial structures, the non-normality of the eigenvalue problem gives rise to large differences between direct and adjoint eigenfunction. This suggests that the instability mechanism cannot be identified from the direct and adjoint eigenfunctions separately $[8,50,82]$. So a practical way is to evaluate the overlap between direct and adjoint eigenfunction [50]. The product of overlap indicates the sensitive region[58, 59], where a spatially base flow modification may alter the stability properties of the flows.

By perturbing the direct eigenvalue problem (2.12), it is obtained that,

$$
\delta(\mathbf{A}-\lambda \mathbf{I}) \hat{\mathbf{u}}=(\delta \mathbf{A}-\delta \lambda \mathbf{I}) \hat{\mathbf{u}}+(\mathbf{A}-\lambda \mathbf{I}) \delta \hat{\mathbf{u}} .
$$

and using the adjoint relation (2.16) leads to a relation between the change induced in the eigenvalue $\delta \lambda$ by the modification of the Jacobian-matrix,

$$
\delta \lambda=\frac{\hat{\mathbf{u}} \delta \mathbf{A} \hat{\mathbf{v}}}{<\hat{\mathbf{u}} \cdot \hat{\mathbf{v}}>} .
$$

If a localized in space perturbation as $\delta \mathbf{A}=\delta\left(x-x_{0}, y-y_{0}, z-z_{0}\right)$ is introduced in the above equation, it is possible to define a function that localizes the spatial positions where the $\lambda$ eigenvalue is most sensible to perturbations of the Jacobian-matrix through small changes in the base flow, enabling the possibility of suppressing instability onsets by small modifications in the base flow, as done experimentally by Strykowski and Sreenivasan [108], which leads to

$$
\delta \lambda=\frac{\|\hat{\mathbf{u}}\|\|\hat{\mathbf{v}}\|}{<\hat{\mathbf{u}} \cdot \hat{\mathbf{v}}\rangle} .
$$

See Appendix A for the derivation of the adjoint operator, the derivation is omitted for simplicity and the adjoint equations will be presented with correct boundary conditions that satisfy a zero bilinear concomitant, the most critical step is deriving proper boundary conditions for the treatment of the bilinear concomitant. Further details can be checked in the literature $[10,50,58,116]$. 


\section{Chapter 3}

\section{Numerical Solution of the Eigenvalue Problem}

In the previous chapter, linear stability theory and adjoint-based sensitivity theory are presented. Different numerical methods to analyze global instability are documented in this Chapter, and alternative matrix-free methods are proposed to solve global instability. The numerical details of time-stepping method implemented in open-source CFD code are presented.

Recent advances in CFD software have provided to the computational fluid dynamics community with a great variety of open-source solvers and tools, such as the Stanford University Unstructured $\left(S U^{2}\right)$ [85], Nek5000 [43], Nektar++[22, 118], FreeFem[56] or OpenFOAM ${ }^{\circledR}[120]$, among many alternatives. From an academic point of view, the major advantage of the use of these numerical solvers against the proprietary software, besides license related issues, is the access to the source code and the possibility of customize it for multiple purposes. In addition, these suits allow the use of multiple pre- and postprocessing tools such as parallel processing or mesh creation/manipulation utilities, whose availability a priori can save significant effort and time in the obtaining of results from numerical simulations.

Two classes of approaches can be used to solve the eigenvalue problem (EVP): matrixforming and time-stepping. The matrix forming strategies give all the information of eigenmodes by providing access to larger subsets of the full spectrum, it costs a large computational memory (RAM memory) due to storage of the large matrices. Time-stepping method provides smaller subsets of the spectrum at the cost of long time integration (CPU time). Both methods have different advantages.

Matrix-forming approach has an inherent advantage in deposing the simple geometry, programming simplicity and flexibility. For the one-dimensional problem, it is straightforward to form any of the linear one-dimensional operators. In two or three dimensional problem, the Kronecker product of matrices can be used for multi-dimensional problems. An additional key advantage of a matrix-forming approach is its flexibility: incompressible or compressible, viscous or inviscid operators may be programmed in a straightforward manner into the same code, thus minimizing the amount of validation and verification work necessary when a new problem is to be tackled.

Alternative time-stepping method may be used due to simply implemented to existed CFD codes. A linearized Navier-Stokes solver are need to be implemented in the DNS code firstly, operation is performed in the same manner as those of the underlying DNS code used. Moreover, time-stepping method is good at tackling complex geometry.

In this chapter, details of implementing direct and adjoint linearized Navier-Stokes solver are presented in OpenFOAM. Time-stepping method, moreover Residual Algorithm, coupled with the previous modified code has been introduced. With an effort, solving EVPs by Arnoldi methods in Nek5000 has been introduced briefly. Since mature version of time-stepping code has been developed by Peplinski et al. [88], in this context, all the work in Nek5000 part has been solved based on this remarkable progress. 


\subsection{Direct and adjoint linearized Navier-Stokes equations}

The development of the direct and adjoint linearized Navier-Stokes equations by modifying the incompressible transient solver of the open-source CFD software OpenFOAM ${ }^{\circledR}$, named icoFoam is shown next. This solver, based on a classical finite volume formulation [42] and a PISO [41, 64, 67] algorithm (Pressure-Implicit with Splitting of Operators), has demonstrated a high quality performance in the recent literature $[15,16,54,102]$. This algorithm and its modifications to create solvers for the linearized and adjoint NavierStokes equation, named dirIcoFoam and adjIcoFoam respectively, are explained here in detail. The key idea of the PISO algorithm is that for small time-steps the pressurevelocity coupling is much stronger than the non-linear convective coupling, therefore it is possible to split the solution into a set of corrections where the pressure is decoupled from the velocity, since the velocity in the momentum equation does not need to be updated for each pressure correction. Although this procedure is not formally necessary to solve the linearized Navier-Stokes equations (LNSE), since these equations are linear, the PISO method of solution can be applied in order to treat in a explicit manner the convective term in which the volume flux contribution of the perturbation velocity needs to be evaluated, as it is detailed next.

A similar notation to Jasak [67] will be employed in what follows. As an example, the semi-discretization of the convective term of the Navier-Stokes using finite volumes in a polyhedron is written as:

$$
\int_{V} \nabla \cdot(\boldsymbol{u} u) d V=\int_{S} u \boldsymbol{u} \cdot \hat{n} d S=\sum_{i} \boldsymbol{u}^{i} \cdot S^{i} u^{i}=\sum_{i} \phi^{i} u^{i}=\mathbf{a}_{\mathbf{P}} u_{P}+\sum_{N} \mathbf{a}_{\mathbf{N}} u_{N}
$$

where the velocity is evaluated on the faces $i$ of the polyhedron $P . S^{i}$ is the normal vector to face $i$ which norm equal to the face area, and subscript $N$ represents the neighbors polyhedron to P. $\phi^{i}$ is the flux across face $i$, and $\mathbf{a}_{\mathbf{P}}$ and $\mathbf{a}_{\mathbf{N}}$ are the function of $\mathbf{u}$. Finally, the flux is obtained by interpolation of the node values adjacent to each surface. Taking this formulation into account, the momentum equation of the Navier-Stokes equation in (2.1b) can be equivalently defined and initialized as:

$$
\boldsymbol{u}=\mathbf{a}_{\mathbf{P}}{ }^{-1} \mathbf{H}(\mathbf{u})-\mathbf{a}_{\mathbf{P}}{ }^{-1} \nabla p,
$$

where ap contains the discretization matrix operator of the implicit terms while $\mathbf{H}$ represents the explicit terms of the velocity. The first two terms of the above equations are represented in line 1 of Listing 3.1 in OpenFOAM ${ }^{\circledR}$ notation, where $\mathrm{f} v \mathrm{vm}$ is the namespace which are defined the implicit version of the operators (ddt, div or laplacian). A momentum predictor is then obtained using the momentum equation and the pressure from a previous step, which in OpenFOAM ${ }^{\circledR}$ notation is written in line 3 of Listing 3.1. 
Listing 3.1: Projection step

fvVectorMatrix UEqn (fvm: :ddt(U)+fvm::div(phi, U) - fvm: : laplacian(nu, ...

Recalling equation (3.2), the explicit terms can be written as

$$
\boldsymbol{u}^{*}=\mathbf{a}_{\mathbf{P}}{ }^{-1} \mathbf{H},
$$

This term is represented in lines 5 and 6 in Listing 3.1, where the functions UEqn.A() and UEqn.H() extract the implicit and explicit terms from equation (3.2). The momentum equation is then written as:

$$
\boldsymbol{u}=\boldsymbol{u}^{*}-\mathbf{a}^{-1} \nabla p,
$$

where the left hand side term is divergence-free and the continuity equation can be applied to obtain the laplacian equation:

$$
\nabla \cdot \boldsymbol{u}^{*}=\nabla \cdot \mathbf{a}_{\mathbf{P}}^{-1} \nabla p
$$

in which the left hand side can be treated explicitly. Next the velocity flux $\phi$ is updated with the new velocity, which permits the obtention of the new pressure field, as seen in lines 8 and 9 in Listing 3.1.

Finally the divergence-free velocity $\boldsymbol{u}$ is corrected with the correct pressure gradient with Equation (3.2) and the last step of the PISO algorithm consists of advancing the time-step. Non-orthogonality effects, boundary conditions corrections and details about the discretization have been omitted for simplicity. This PISO algorithm is summarized in Algorithm 1. This algorithm can be easily modified in order to solve the direct and adjoint linearized Navier-Stokes equations. It is trivial to observe from (2.4) and (A.6) that the main difference with the full Navier-Stokes equations are the additional advection terms and the different signs in case of the adjoint equations. Since the terms related with the base flow are constant, and the predictor fluxes of the perturbation are constructed with the perturbation velocity from previous step, the additional advection term of direct and adjoint equations can be formed in a explicit manner, thus the equations to be solved are equivalent to the non-linear case. In other words, the base flow advection term can be treated as a source term. Therefore, the dirIcoFoam and adjIcoFoam solvers for the solution of the temporal evolution of the linearized direct and adjoint Navier-Stokes equations (2.4) and (A.6) can be constructed by modifying the matrix UEqn including the new advection terms without further modifications. For dirIcoFoam this is represented in Listing 3.1, where $\mathrm{U}$ refers now to the perturbation, UB to the base flow and $\mathrm{fvc}$ is the namespace which are defined the explicit version of the operator (div).

Listing 3.2: Correction step in dirIcoFoam 


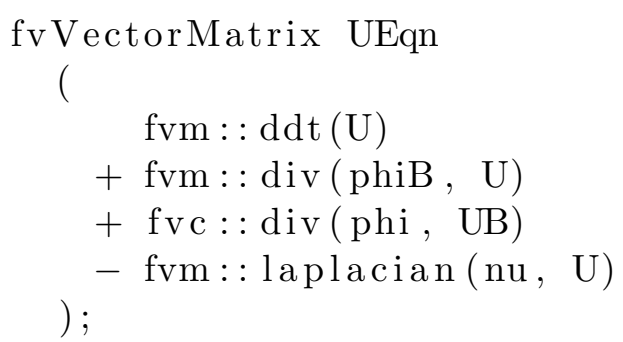

Similarly, the adjoint equation solver dirIcoFoam is represented in Listing 3.3, where V refers to the adjoint perturbation. Note that special treatment of the temporal scheme is required for the backwards temporal integration of the adjoint equations.

Listing 3.3: Correction step in adjIcoFoam

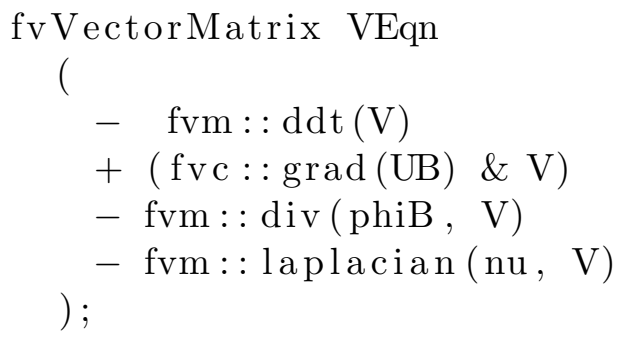

\subsection{Residual Algorithm}

Residual algorithm [112, 114, 119] has been employed to validate the transient behavior of the solver. In the case of steady state flow, when the govern equations of motion (2.1) start from a random initial condition, match as the time integration, the path of the flow quantity $\mathbf{u}$ to the steady state solution is charged by the damping of the least stable flow eigenmodes. As such, during the time prior to convergence, the residual is governed by only the leading damped linear global mode. In the particular case of a steady leading mode, if the time-accurate integration is close to convergence, the decomposition (2.3) is valid, the corresponding damping ratio can be extracted from the logarithmic derivative of the signal, which reads

$$
\lambda_{r}=\frac{\ln \left(\frac{\varepsilon(t+\Delta t)}{\varepsilon(t)}\right)}{\Delta t},
$$

$\lambda_{r}$ is identified as the damping ratio corresponding to the global modes and

$$
\varepsilon(t)=|q(x, y, z, t)-\bar{q}|,
$$

$q$ is any flow quantity obtained from the DNS transient data.

In the case of a traveling leading mode, in order to extract frequency $\lambda_{i}$, fast Fourier transform is performed to the time trace of flow quantity in the finite convergence range.

For the least stable eigenmode, it has largest damping/growth ratio and dominant oscillation than secondary eigenmode. Damping/growth ratio and circular frequency can be settled using aforementioned methods. Residual algorithm developed by Theofilis [111] is used to determine the spatial eigenmode $\hat{\mathbf{u}}$. This consists of writing solution (2.11) at two different times $t_{1}$ and $t_{2}$ with two unknown parameters $\hat{\mathbf{u}}_{r}$ and $\hat{\mathbf{u}}_{i}$

$$
\tilde{\mathbf{u}}\left(x, y, z, t_{1}\right)=\left(\hat{\mathbf{u}}_{r}(x, y, z) \cos \lambda_{i} t_{1}-\hat{\mathbf{u}}_{i}(x, y, z) \sin \lambda_{i} t_{1}\right) e^{\lambda_{r} t_{1}}
$$




$$
\tilde{\mathbf{u}}\left(x, y, z, t_{2}\right)=\left(\hat{\mathbf{u}}_{r}(x, y, z) \cos \lambda_{i} t_{2}-\hat{\mathbf{u}}_{i}(x, y, z) \sin \lambda_{i} t_{2}\right) e^{\lambda_{r} t_{2}}
$$

leading to a easily solvable linear system. Since this method is solely based on power iterations, the present algorithm can only provide the structural sensitivity of the leading eigenvalue of the flow, which is the responsible for the onset on the instability. In addition, this method is equivalent to the temporal integration, so it converges as $\sim e^{\left|\lambda_{1}-\lambda_{2}\right| \tau}$, thus this algorithm is very effective when a gap between first and second eigenvalue is expected in the spectrum. In addition, because of this, the computational resources employed in terms of CPU time in the analysis can be significantly smaller to those required in the obtaining of the steady base flow using a transient method. Further modifications based on the update of the eigenvalue shift $\lambda_{s}$ at each step by making use of the direct adjoint relationship (2.19), as envisaged by Giannetti and Luchini [50] in a shift-invert matrixforming framework, can be introduced in the algorithm.

Alternatively, Krylov-subspace projection methods, such as those described in Tuckerman and Barkley [116] and Barkley et al. [10], are straight forward to implement with the dirIcoFoam and adjIcoFoam solvers. Particularly, this is the prefered method in case the obtention of a larger subset of the spectrum is required. Algorithm 3 shows the implementation of this methodology employing the solvers dirIcoFoam and adjIcoFoam.

Algorithm 1 PISO algorithm

S1: Set initial conditions

S2: Time-step iteration $\left(t=0, \ldots, t_{f}\right)$

T1: Obtain momentum predictor by solving momentum equation using mass flux $\phi$ from previous velocity and pressure $\mathbf{a}_{\mathbf{P}} \mathbf{u}^{*}=\mathbf{H}-\nabla p_{n-1}$

T2: PISO Iteration $\left(i=1, \ldots, N_{P I S O}\right)$

P1: Compute volume fluxes

P2: Solve the pressure equation

P3: Correct the velocity with new pressure field

P4: Update boundary conditions.

S3: Advance time-step 
Algorithm 2 Sensitivity analysis with direct and adjoint power iteration

A1. Direct problem: Solve the linearized Navier-Stokes equations

B1. Initial Condition: Set $\tau, \mathbf{u}^{\prime}(0), \overline{\mathbf{u}}$

B2. Call dirIcoFoam: $\mathbf{u}^{\prime} \leftarrow e^{\mathbf{A} \tau} \mathbf{u}^{\prime}(0)$

C1. Fit resisdual data via least-squares to obtain $\lambda_{r}, \lambda_{i}$ :

C2. Apply residual algorithm to obtain $\hat{u}_{\mathrm{r}}$ and $\hat{\mathrm{u}}_{\mathrm{i}}$ :

A2. Adjoint problem: Solve the adjoint linearized Navier-Stokes equations

B1. Initial Condition: Set $\tau, \mathbf{v}^{\prime}(0), \overline{\mathbf{u}}$

B2. Call adjIcoFoam: $\mathbf{v}^{\prime}(0) \leftarrow e^{\mathbf{A}^{*} \tau} \mathbf{v}^{\prime}$

C1. Fit residual data via least-squares to obtain $\lambda_{r}, \lambda_{i}$ :

C2. Apply residual algorithm to obtain $\hat{v}_{\mathbf{r}}$ and $\hat{\mathbf{v}}_{\mathbf{i}}$ :

A3. Structural sensitivity: Compute $\delta \lambda(x, y)=\frac{\left|\hat{\mathbf{v}}_{1}\right|\left|\hat{\mathbf{v}}_{1}\right|}{\hat{\mathbf{v}}_{1} \cdot \hat{\mathbf{u}}_{1}}$ 
Algorithm 3 Sensitivity analysis following Barkley et al. [10] methodology

A1. Direct problem: Solve direct EVP

B1. Initial condition direct problem: Set $m, \epsilon$ and $\mathbf{u}_{1}^{\prime}$

B2. Arnoldi iteration: Perform until convergence $(l=1, \ldots, m)$,

C1. Call dirIcoFoam: $\mathbf{u}_{l}^{\prime} \leftarrow e^{\mathbf{A} \tau} \mathbf{u}_{l}^{\prime}$

C2. Gram-Schmidt orthonormalization: $(i=1, \ldots, l)$

D1. Form Hessenberg matrix $h_{i l}=\mathbf{u}_{i}^{\prime T} e^{\mathbf{A} \tau} \mathbf{u}_{l}^{\prime}$

D2. Orthogonalize $\mathbf{u}_{l+1}^{\prime}=e^{\mathbf{A} \tau} \mathbf{u}_{l}^{\prime}-\sum_{i=1}^{j} h_{i l} \mathbf{u}_{i}^{\prime}$

D3. Normalize $h_{l+1, l}=\left\|\mathbf{u}_{l+1}^{\prime}\right\|, \mathbf{u}_{l+1}^{\prime}=\frac{\mathbf{u}_{l+1}^{\prime}}{h_{l+1, l}}$

B3. QR: Perform eigenvalue decomposition of the $m \times m$ matrix $\mathbf{H}$ and undo exponential transformation.

A1. Adjoint problem: Solve adjoint EVP

B1. Initial condition adjoint problem: Set $m, \epsilon$ and $\mathbf{v}_{1}^{\prime}$

B2. Arnoldi iteration: Perform until convergence $(l=1, \ldots, m)$,

C1. Call adjIcoFoam: $\mathbf{v}_{l}^{\prime} \leftarrow e^{\mathbf{A}^{*} \tau} \mathbf{v}_{l}^{\prime}$

C2. Gram-Schmidt orthonormalization: $(i=1, \ldots, l)$

D1. Form Hessenberg matrix $h_{i l}^{*}=\mathbf{v}_{i}^{\prime T} e^{\mathbf{A}^{*} \tau} \mathbf{v}_{l}^{\prime}$

D2. Orthogonalize $\mathbf{v}_{l+1}^{\prime}=e^{\mathbf{A}^{*} \tau} \mathbf{v}_{l}^{\prime}-\sum_{i=1}^{j} h_{i l}^{*} \mathbf{v}_{i}^{\prime}$

D3. Normalize $h_{l+1, l}^{*}=\left\|\mathbf{v}_{l+1}^{\prime}\right\|, \mathbf{v}_{l+1}^{\prime}=\frac{\mathbf{v}_{l+1}^{\prime}}{h_{l+1, l}^{*}}$

B3. QR: Perform eigenvalue decomposition of the $m \times m$ matrix $\mathbf{H}^{*}$ and undo exponential transformation.

A3. Structural sensitivity: Compute $\delta \lambda=\frac{\left\|\hat{\mathbf{v}}_{1}\right\|\left\|\hat{\mathbf{u}}_{1}\right\|}{\hat{\mathbf{v}}_{1} \cdot \hat{\mathbf{u}}_{1}}$ 


\subsection{Time-stepping method implemented in Nek5000}

The non-linear Navier-Stokes equations as well as their linear counterparts are partial differential equations, they need to be spatially and temporally discretized in order to be solved numerically. The open-source code Nek5000 is based on high order numerical scheme spectral element method [87], it combines the generality of the finite element method with the accuracy of spectral techniques. Moreover spectral elements mesh relies on two different grid levels: finite element grid and spectral collocation points $n$ within each element, the accuracy of spatial-discretization $p$ depends on $p=n-1$. A stagged grid technique is used for the velocity and pressure spatial-discretization, hence pressure boundary conditions are omitted. Semi-implicit scheme $B D F_{k} / E X T_{k}$ is used for temporal discretization. More details can be found in [69].

Linear stability analysis introduces Jacobian matrix $\mathbf{A}$ in (2.5), which contains the key stability property of the flow. So, the core task becomes how to solve eigenvalue problems from (2.5) and obtain eigenpairs $(\lambda, \hat{\mathbf{u}})$. while, solving the large eigenvalue problems by direct methodology is still a challenge job, especially for three-dimensional complex geometry. So time-stepping method has been taken as one of the efficient methodology, it has been introduced in the mid 90's[38] and popularized since 2009[8].

The initial value problem (2.5) can be transformed to the following form:

$$
\tilde{\mathbf{u}}_{1}(\tau)=e^{\mathbf{A} \tau} \tilde{\mathbf{u}}_{\mathbf{0}}
$$

where $\tilde{\mathbf{u}}_{\mathbf{0}}$ is the arbitrary initial perturbation which arranged as the vector and assume to be of unit norm $\left\|\tilde{\mathbf{u}}_{\mathbf{0}}\right\|=1$, integration time is $\tau$.

The eigenpairs of $\mathbf{A}$ contain the information of instability of the fluid model which can not be solved directly from (3.9). However, the iteration method for calculating the leading eigenparis of matrix exponential $e^{\mathbf{A} \tau}$ is practical, for convenience, denote $e^{\mathbf{A} \tau}$ as $\boldsymbol{\Sigma}$. Moreover, their eigenpairs of $\mathbf{A}$ and $\boldsymbol{\Sigma}$ have a temporal exponential relationship.

$$
\begin{array}{ccc}
\mathbf{A} & \boldsymbol{\Sigma}, \\
(\lambda, \hat{\mathbf{u}}) & \underset{\text { exponential }}{\stackrel{\text { temporal }}{\longrightarrow}}(\sigma, \hat{\boldsymbol{u}})
\end{array}
$$

and

$$
\lambda=\frac{\log (\sigma)}{\tau}, \quad \hat{\mathbf{u}}=\hat{\boldsymbol{u}} .
$$

Hence, eigenvalue problems become solving indirect temporal propagator matrix $\boldsymbol{\Sigma}$ instead of Jacobian Matrix A. The spectrum transformation is required for the conversion of the leading eigenvalues into the dominant eigenvalues, which are the ones that the Arnoldi method can recover. Because of exponential transformation, figure 3.2 shows the temporal exponential transformation of the spectrum, it can be seen how the leading eigenvalue of the Jacobian matrix $\mathbf{A}$ becomes the dominant in the temporal propagator matrix $\sigma$, at the same time, the dominant eigenvalues are shifted to zero.

The approach for solving eigenvalue problem is resorted to Arnoldi method. Arnoldi method is an important iterative methods for eigenvalue algorithm. The Arnoldi iteration [4] uses the stabilized Gram-Schmidt process to produce a sequence of orthonormal vectors, $q_{1}, q_{2}, q_{3}, \ldots$, called the Arnoldi vectors, such that for every Krylov dimension $k$, the 


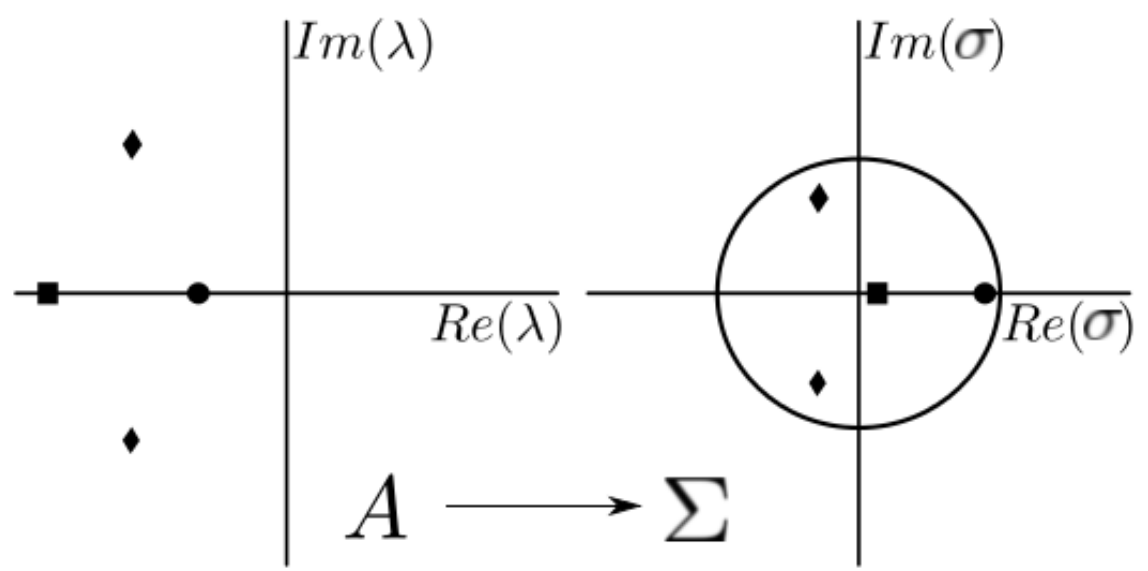

Figure 3.1: Temporal exponential transformation [52].

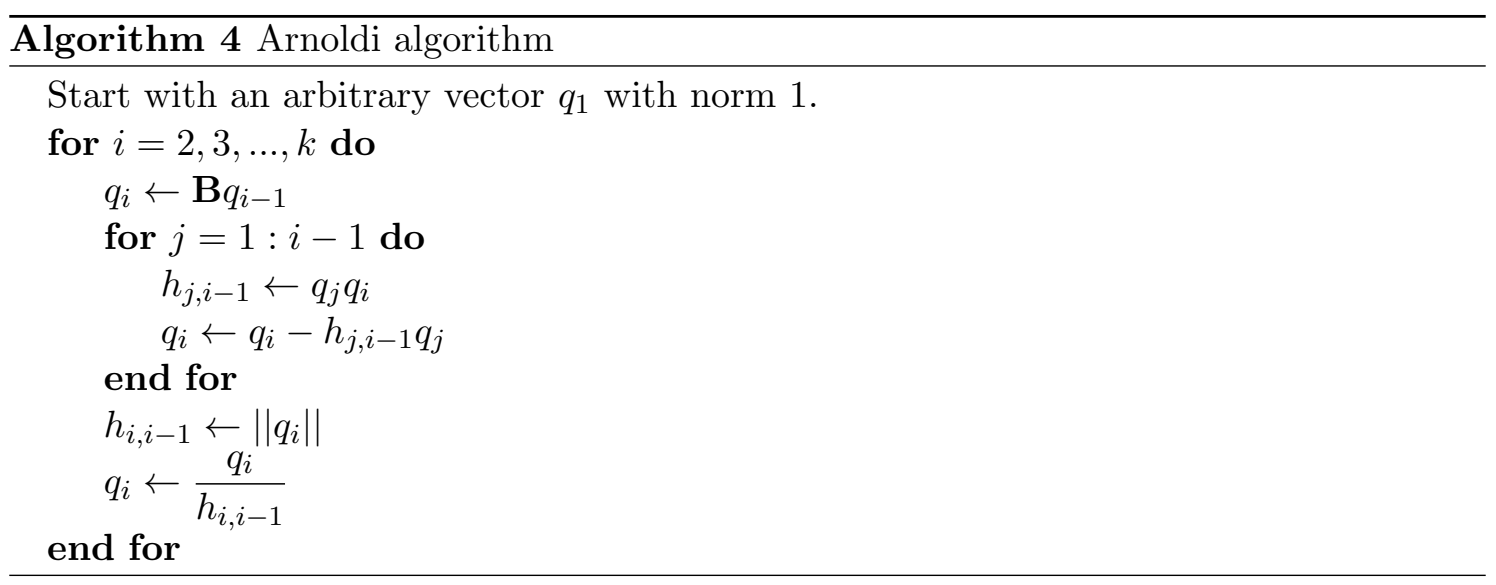


vectors $q_{1}, \ldots, q_{k}$ span the Krylov subspace. Explicitly, the prototype of Arnoldi algorithm is presented in algorithm 4.

One of the most challenging tasks of the proposed time-stepper method is the corrected selection parameters: integrate time $\tau$ and Krylov subspace dimension $k$. Bagheri et al. [7] gave the quantitative criteria for selecting integrate time. Integrate time $\tau$ is a balance value between Nyquist frequency and convergence of numerical simulation. Since every basis vector of the Krylov subspace is created by a simulation running in time iteration $\tau$, the time integration should make sure the created Krylov subspace reflects the physical structures of the flow. Two guidelines can be considered as the reference to choose properly time integration, $\tau$ must be small enough to include at least two sampling points in one period of the highest-frequency mode $\left(\tau<T=2 \pi / \lambda_{i, \max }\right)$; the angular frequency of the global modes must be contained in the range of Nyquist frequency $\omega_{N y q u i s t}=\pi / \tau$.

$$
\lambda_{i, \max } \in[0, \pi / \tau] .
$$

Usually, the frequency spectrum of flow field from DNS can be taken as the reference for time-stepping method.

Note that one does not know beforehand how large the Krylov subspace has to be to converge to the desired number of eigenvalues. Because the convergence of Arnoldi method depends very much on initial condition. The classic Arnoldi method, initialized of random vector, must spend large dimensional of subspace in order to recovery several leading eigenvectors within satisfied tolerance. So it is quite less efficiency for the fully three-dimensional problem. Implicit restarting Arnoldi method uses a refined initial condition and restarts the Arnoldi factorization with this new vector instead, it shrinks the dimensions of the Krylov subspace, the minimal Krylov subspace $k$ can be 2 times of the desired eigenmodes.

\subsection{Steady state solution}

Steady base flow solution of Navier-Stokes equations is a pre-requisite to linear stability studies. Especially when the flow under consideration is globally unstable, it is impossible to using time-marching method to acquire the steady solution of non-linear Navier-stokes. Several conservation approaches have been applied for the limited cases, such as enforcing symmetries in the system ( $2 \mathrm{D}$ flow over a circular cylinder), artificially setting the velocity component in certain directions to zero and fixed point of the equations. The more general approach is Newton iteration methods which relies on the computation of a Jacobian matrix. It becomes quite expensive and inefficient when dealing with fully three-dimensional Navier-Stokes equations.

Åkervik et al. [1] overcame this problem by introducing an iteration method known as the selective frequency damping method. This technique is adapted from large eddy simulation techniques [93, 94], and it enables a damping of oscillations of the unsteady part of the solution using a temporal low-pass filter, this is achieved by adding a forcing term to the right-hand side of the Navier-Stokes equations and extending the system with an extra equation for the filtered state $\overline{\mathbf{u}}$. The extended system is governed by the following equations.

$$
\nabla \cdot \mathbf{u}=0
$$




$$
\begin{aligned}
\frac{\partial \mathbf{u}}{\partial t}+\mathbf{u} \cdot \nabla \mathbf{u} & =-\nabla p+R e^{-1} \nabla^{2} \mathbf{u}-\chi(\mathbf{u}-\overline{\mathbf{u}}) \\
\frac{\partial \overline{\mathbf{u}}}{\partial t} & =\omega_{c}(\mathbf{u}-\overline{\mathbf{u}})
\end{aligned}
$$

where $\chi$ is control coefficient and $\omega_{c}$ being filter width [93]. The choice of two open parameters is critical for the computation. As the guideline, $\chi$ is chosen to be twice the growth rate of the targeted unstable instability mode, and the cutoff frequency $\omega_{c} \approx \frac{1}{2 \cdot \omega_{\text {uns }}}$ in order to make sure the unstable disturbances frequency is within the damped region. It is noteworthy that this technique is failed for the case that the leading unstable global mode is stationary. In that situation, investigators may resort to the conservation approaches reminded before. 


\section{Chapter 4}

\section{Validations}

In the previous chapter time-stepping method is proposed to solve global instability problems and adjoint-based sensitivity analysis for the detection of flow properties. The code modification of direct and adjoint linearized solvers are presented using OpenFoam and Nek5000. These methods and their validations to solve linear global instability and structural sensitivity are presented in this chapter. The validations are separated in two parts.

Residual algorithm is performed on the two-dimensional and three-dimensional open cavity with the aspect ration $\mathrm{L} / \mathrm{D}=2$. The spanwise length of three-dimensional open cavity is the approximately one cavity depth, $L / \Lambda=1.1$. In the case of two-dimensional open cavity, damping rate and circular frequency of the leading eigenmode are detected at $R e=1400$ and $R e=1900$, the results are in good agreement with the work from De Vicente [33]. Moreover, the structural sensitivity results have been revealed in both cases. The obtained damping rate and frequency of the least stable eigenmode in threedimensional open cavity are compared with the results from Brès and Colonius [19]. The visualized spatial structure of direct and adjoint global mode are confined inside the cavity. Additionally, structural sensitivity of three-dimensional open cavity has been identified. More details about these results and the alternative matrix free methods have been published in [78].

The intention of this work was to undertake all the thesis with OpenFoam. However, it become computationally unaffordable for the required resolutions in three-dimensional problems. Hence, we must leave the OpenFoam and turned to high-order numerical methods Nek5000. Luckily, the time-stepping method has been employed in this open source code by Peplinski et al. [88]. Four selected cases from two-dimensional wall-bound to three-dimensional open flows have been verified using this modified code: two-dimensional lid-driven cavity [53], two-dimensional circular cylinder [50], two and half dimensional open cavity [27] and three-dimensional lid-driven cavity [54].

\subsection{Validation in OpenFoam}

\subsubsection{Two-dimensional open cavity flow}

The cavity configuration and flow conditions are controlled by the following parameters: the ratio of the cavity length to depth $L / D$, the Reynolds number depending on the cavity depth $R e_{D}=\frac{U D}{\nu}$, the ratio of the cavity length to the initial boundary layer momentum thickness at the leading edge of the cavity $L / \theta$ and the boundary layer displacement thickness $\delta^{*}$. Reynolds number depending on the displacement thickness $R_{\delta^{*}}$ is also employed in order to control the inlet boundary layer thickness. All of the parameters involved with the Blasius boundary layer are measured in the upstream lip of the cavity, the initial $R e_{\delta^{*}}$ is the approximately the same in the cases of $R e_{D}=1400$ and $R e_{D}=1900$, as shown in the Table 4.1. The sensitive results will show the most unstable region changes 
as the $R e_{D}$ increases.

\begin{tabular}{cccc}
\hline$R e_{D}$ & $L / \theta$ & $\delta^{*}$ & $R e_{\delta^{*}}$ \\
\hline 1400 & 51.948 & 0.0997 & 139.58 \\
\hline 1900 & 70.572 & 0.0734 & 139.46 \\
\hline
\end{tabular}

Table 4.1: Parameters of the two-dimensional open cavity with aspect ratio $\mathrm{L} / \mathrm{D}=2$.

A variation of the Richardson [95] extrapolation generalized by Roache [96] is introduced for the mesh refinement study, Sanmiguel-Rojas et al. [102] successfully used this method for studying the grid independent of the flow past an axisymmetric body. The grid convergence index can be defined as

$$
G C I_{j, j+1}=3 \times\left|\frac{u_{j}-u_{j+1}}{u_{j+1}\left(l^{n}-1\right)}\right| \times 100,
$$

where $u_{j}$ is the variable discretized on mesh $j, j+1$ is finer than $j$, n represents the order of the convergence rate of this method which is

$$
n=\log \left(\frac{u_{j}-u_{j+1}}{u_{j+1}-u_{j+2}}\right) / \log (l) .
$$

As shown in Table 4.2, three different resolutions with refinement ratio $l=1.5$ in each direction is employed. The streamwise velocity $u$ at point $(2.0,0.0)$ which located at the shear layer region of the cavity has been probed in the two-dimensional open cavity at $R e_{D}=1400$. Notice that as the grid is refined, the grid convergence index decreases, $G C I_{1,2}>G C L_{2,3}$. So the mesh M2 with 24280 cells is enough to accurately predict the flow in the open cavity at $R e_{D}=1400$. Here we used M2 as the resolution for the two-dimensional and the following three-dimensional computation, the mesh information is showed in the Figure 4.1.

\begin{tabular}{cccc}
\hline Mesh & cells numbers & $u(2.0,0.0)$ & $G C I_{j+1, j}(\%)$ \\
\hline M1 & 10878 & 0.20243 & \\
\hline M2 & 24280 & 0.20338 & 0.82019 \\
\hline M3 & 54136 & 0.20373 & 0.30266 \\
\hline
\end{tabular}

Table 4.2: Grid convergence study based on $u$ velocity for the two-dimensional open cavity at $\mathrm{Re}=1400$ using three meshes: M1 coarse, M2 medium and M3 fine. Grid convergence index obtained with $l=1.5$ and $n=1.25$.

Four types of boundary conditions are employed to solve two-dimensional incompressible Navier-Stokes equations. The velocity of inlet is uniform $\mathbf{u}=(u, v)=(1,0)$, the same as the far-field boundary condition. Neumann velocity boundary condition is used in the outlet and wall $\mathbf{u}=(u, v)=(0,0)$. The boundary condition and domain size for the outflow in an open-flow problem to the sensitivity research are important issues. As documented in the previous work of optimal growth analysis from Barkley et al. [10], the computational domain for an optimal growth computation must have considerably long inflow and outflow lengths in order to avoid the non-negligible amplitude of perturbation in the outlet, that is the reason we give up the short upstream computational domain by employing Blasius profile as the inlet boundary condition. The summary of the boundary 
4.1. Validation in OpenFoam

\begin{tabular}{cccc}
\hline BCs & Base flow (DNS) & Direct modes & Adjoint modes \\
\hline Inlet & $\mathbf{u}=(1,0), \frac{\partial p(x, y)}{\partial n}=0$ & $\mathbf{u}=(0,0), \frac{\partial p(x, y)}{\partial n}=0$ & $\mathbf{u}=(0,0), \frac{\partial p(x, y)}{\partial n}=0$ \\
\hline Wall & $\mathbf{u}=(0,0), \frac{\partial p(x, y)}{\partial n}=0$ & $\mathbf{u}=(0,0), \frac{\partial p(x, y)}{\partial n}=0$ & $\mathbf{u}=(0,0), \frac{\partial p(x, y)}{\partial n}=0$ \\
\hline Farfield & $\mathbf{u}=(1,0), \frac{\partial p(x, y)}{\partial n}=0$ & $\mathbf{u}=(0,0), \frac{\partial p(x, y)}{\partial n}=0$ & $\mathbf{u}=(0,0), \frac{\partial p(x, y)}{\partial n}=0$ \\
\hline Outlet $^{\prime}$ & $\frac{\partial \mathbf{u}}{\partial n}=0, p(x, y)=0$ & $\frac{\partial \mathbf{u}}{\partial n}=0, p(x, y)=0$ & $\mathbf{u}=(0,0), p(x, y)=0$ \\
\hline Periodic $^{\dagger}$ & & $\mathbf{u}=(0,0), \frac{\partial p(x, y)}{\partial n}=0$ & $\mathbf{u}=(0,0), \frac{\partial p(x, y)}{\partial n}=0$ \\
\hline
\end{tabular}

Table 4.3: The summary of the Boundary conditions of the open cavity simulation with OpenFOAM, Periodic ${ }^{\dagger}$ is special for the spanwise periodic open cavity case with the base flow from the spanwise extent of the $2 \mathrm{D}$ base flow.

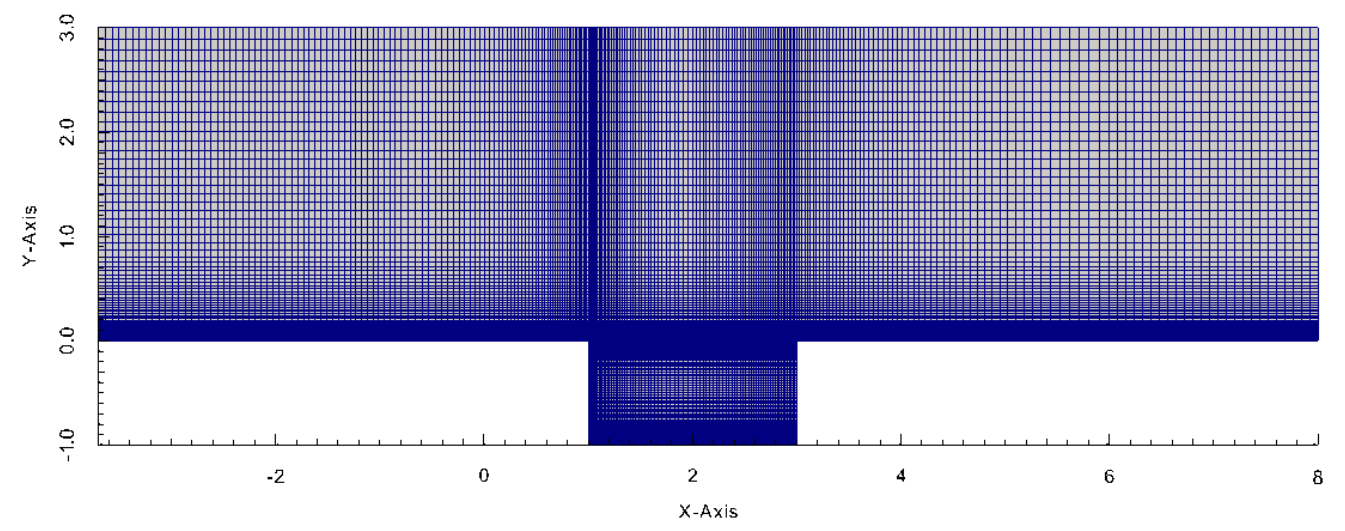

Figure 4.1: Two-dimensional open cavity mesh.

conditions (BCs) of OpenFOAM runs used in the DNS, linear instability simulation and sensitivity simulations are shown in table 4.3.

Figure 4.2 shows the time evolution of the residual of kinetic energy from DNS of the two-dimensional open cavity at $R e_{D}=1400$, the constant of slope corrsponds to the damping rate of the least stable stationary mode $\lambda=0.0179$, where $\Delta E=E(t)-E_{0}, E_{0}$ is the convergence value of kinetic energy. The value is good agreement with the spectrum which getting from matrix-forming method by Vicente[33].

The recovered eigenvalues calculated by residual algorithm[111, 114] with three different solvers can be seen in Table 4.4.

Figure 4.3 shows the corresponding eigenfunctions of these least stable eigenvalues. At $R e=1400$, we can see in the Figure 4.3, the least stable mode is stationary. The direct and adjoint velocity fields are confined inside of the cavity. It is postulated that the instability properties in this flow condition are related to the recirculated region inside cavity. Brès and Colonius [19] have investigated the subcritical condition $R e=1500$ for two-dimensional open cavity flow and point out the shear layer mode damps fast, the oscillation frequency can only be measured at early times which prove the stationary instability mode are more aggressive. The sensitivity result is considered in the good agreement with the reference observations, as shown in the Figure 4.5(a). Figure 4.4 displays the direct and adjoint eigenfunction corresponding to shear layer mode at $R e=1900$. The wave-shedding in the downstream of the cavity has been observed in the direct obtained global mode, while the series of wake shedding instability obtained in the upstream of the cavity of the 


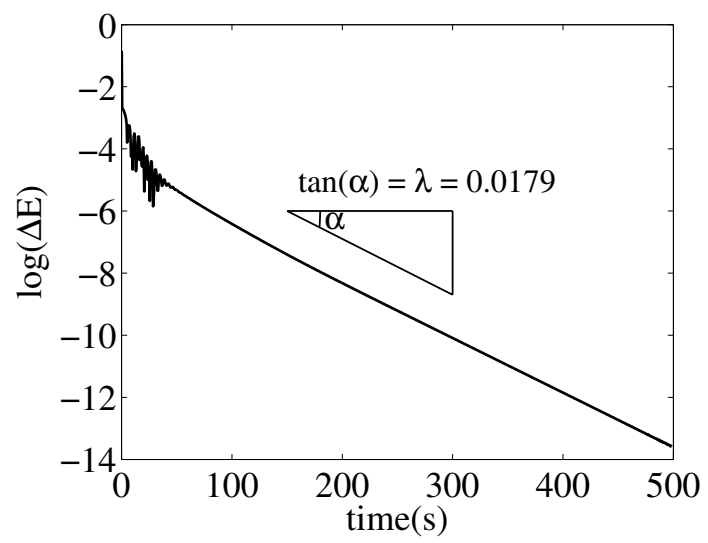

Figure 4.2: The time evolution of kinetic energy residual of the two-dimensional open cavity at $\mathrm{Re}=1400$ by direct numerical simulation.

adjoint case. This is different from the previous cast $R e=1400$. The structural sensitivity is visualized in figure 4.5(b), a clearly shear layer instability has been revealed, which is consistent with the literature.

\begin{tabular}{ccc}
\hline \multirow{2}{*}{ Runs } & $R e=1400$ & $R e=1900$ \\
\cline { 2 - 3 } & $\lambda_{r}+\mathrm{i} \cdot \lambda_{i}$ & $\lambda_{r}+\mathrm{i} \cdot \lambda_{i}$ \\
\hline DNS & $-0.0179+\mathrm{i} \cdot 0.000$ & $-0.0123+\mathrm{i} \cdot 0.5001$ \\
\hline Linear instability & $-0.0175+\mathrm{i} \cdot 0.000$ & $-0.0124+\mathrm{i} \cdot 0.5001$ \\
\hline Adjoint linear instability & $0.0182+\mathrm{i} \cdot 0.000$ & $0.0124+\mathrm{i} \cdot 0.5002$ \\
\hline
\end{tabular}

Table 4.4: The eigenvalues of the least stable mode get by residual algorithm for two-dimensional open cavity flow at $R e=1400$ and $R e=1900$ with direct numerical simulation (icoFoam), linear instability analysis (dirIcoFoam) and adjoint linear instability analysis (adjIcoFoam).

\subsubsection{Spanwise periodic open cavity flow}

A open cavity flow homogeneous in the spanwise direction is simulated in this work using the three-dimensional linearized and adjoint Navier-Stokes equations. The base flow is composed by two-dimensional steady base flow and a Fourier expansion in the homogeneous direction. To determine the least-damped eigenvalue, the residual algorithm and L2 fitting routines is applied to the velocity history. The flow conditions and cavity configuration are controlled by: cavity length and depth ratio $L / D$, spanwise extent $\Lambda$, the Reynolds number based on the cavity depth $R e=U D / \nu$ and the Reynolds number based on the boundary layer displacement thickness $R e_{\delta^{*}}=U \delta^{*} / \nu$ at the leading lip of cavity. In order to keep the laminar flow when it reaches the cavity, the upstream length of cavity is limited by $R e_{\delta^{*}}$, which needs to be smaller than critical Reynolds number $\operatorname{Re}_{\delta^{*}}$ crit $\approx 520$ [105]. Also downstream, after the cavity, $R e_{\delta^{*}}$ should also keep less than $R e_{\delta^{*}}$ crit in order to avoid Tollmien-Schlichting waves. All the parameters are sketched in the Figure 4.6(a), and the values of the flow parameters are listed in table 4.5.

The mesh convergence is the one of the most critical parts for the accurate computation. In this work a resolution of $171 \times 45 \times 15$ grids for the upper domain of cavity and $54 \times 53 \times 15$ 


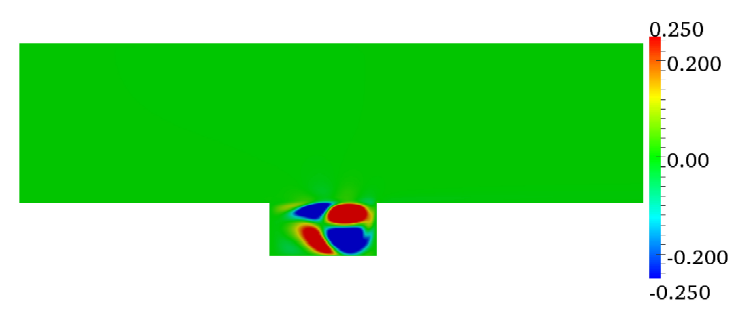

(a) $\hat{u}$

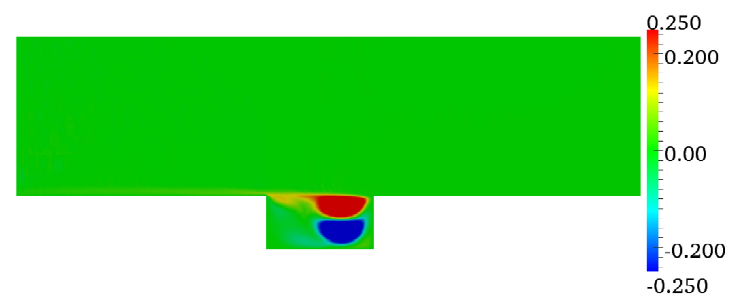

(c) $\hat{u}^{*}$

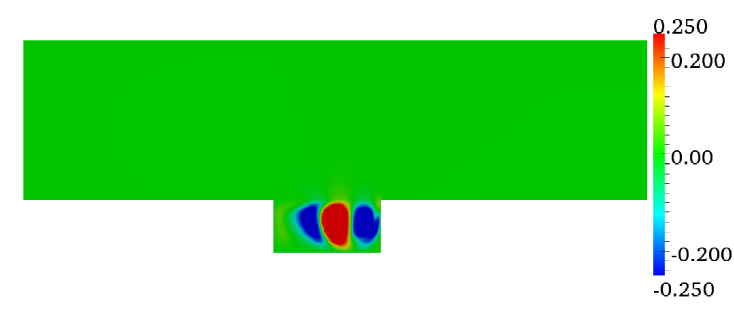

(b) $\hat{v}$

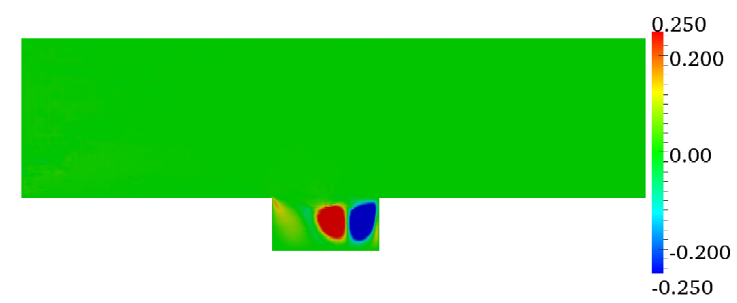

(d) $\hat{v}^{*}$

Figure 4.3: The real part of direct eigenmode $\hat{u}, \hat{v}$ and the real part of adjoint eigenmode $\hat{u}^{*}, \hat{v}^{*}$ of the open cavity at $R e=1400$.

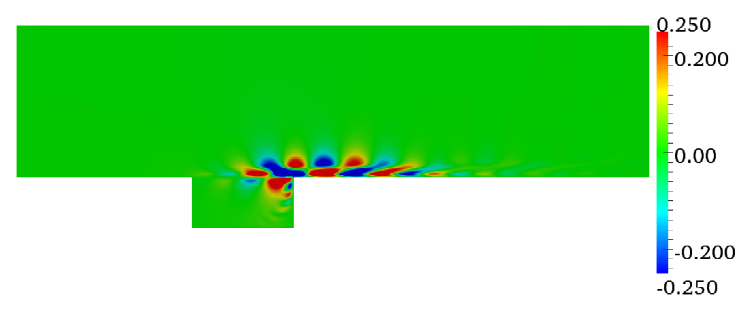

(a) $\hat{u}$

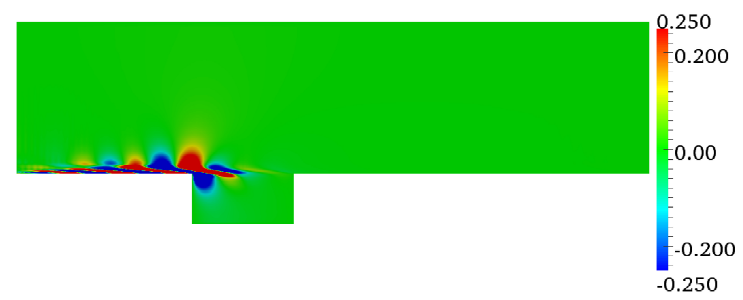

(c) $\hat{u}^{*}$

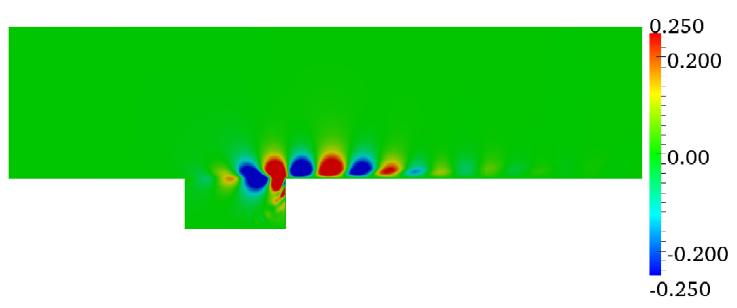

(b) $\hat{v}$

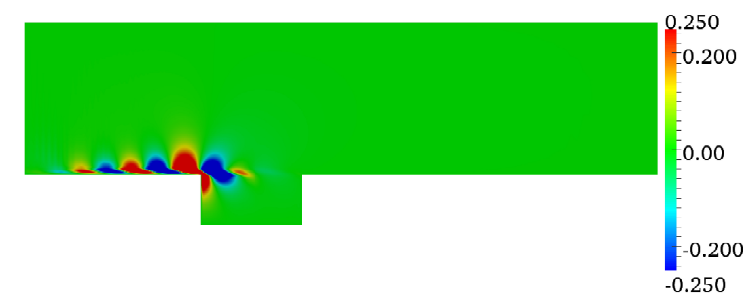

(d) $\hat{v}^{*}$

Figure 4.4: The real part of direct eigenmode $\hat{u}, \hat{v}$ and the real part of adjoint eigenmode $\hat{u}^{*}, \hat{v}^{*}$ of the open cavity at $R e=1900$.

\begin{tabular}{cccccc}
\hline $\mathrm{L} / \mathrm{D}$ & $\Lambda / D$ & $R e$ & $L / \theta$ & $\delta^{*}$ & $R e_{\delta^{*}}$ \\
\hline 2 & 1.1 & 1400 & 51.9488 & 0.0997 & 139.59
\end{tabular}

Table 4.5: Parameters of the open cavity. 


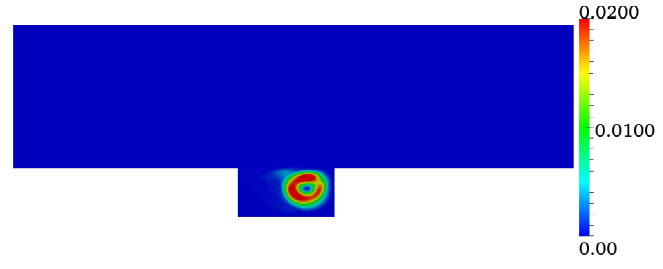

(a) $\delta \lambda(x, y)$

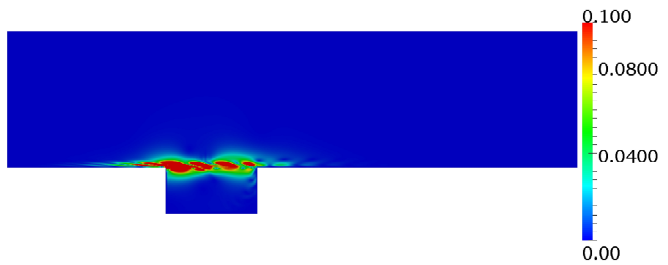

(b) $\delta \lambda(x, y)$

Figure 4.5: The sensitivity functions $\delta \lambda(x, y)$ of the two-dimensional open cavity at $R e=1400$ and $R e=1900$.

degrees of freedom for the cavity domain were considered. Mesh stretching, applied in the wall-normal direction, accumulates the grids near the shear layer in order to reproduce the physical properties as precise as possible.

Five types of the boundary conditions are employed for the direct and adjoint simulations, as summary in the table 4.3. In the adjoint instability case, the size of the computation domain must be a sufficient large as Barkley et al. [10] explained, the perturbations reaches the outlet boundary with non-negligible amplitude will wash the whole computational domain.

We used the icoFoam solver to calculate the two dimensional base flow. For the two dimensional open cavity flow, the shear layer mode is the most important phenomena, and it has been validated by comparing the shear layer spreading rate $d \delta_{\omega} / d x$, where $\delta_{\omega}=U /(d U / d y)_{\max }[101]$ along the shear layer of the cavity, Sarohia [104] found that the spreading was approximately linear, as $L / \theta$ increased from 52.5 to $105.2, d \delta_{\omega} / d x$ increase from 0.025 to 0.088 . In this simulation the value of spreading rate is 0.0482 at $L / \theta=51.948$, which is close to the one found by Rowley et al. [101].

Three dimensional instability results are shown in Table 4.6, the growth/damping rate $\lambda_{r}$, frequency $\lambda_{i}$ and Strouhal number $S t=\lambda_{i} D / 2 \pi U$ are in good agreement with the previous work from Brès and Colonius [19].

Figure 4.6(b) shows the modulus of the velocity $|\hat{\mathbf{u}}|$ of the direct mode at $R e=1400$. It corresponds to the unstable configuration because of the positive growth rate. The direct mode rotates around the primary vortex in the cavity. The three-dimensional instability is a centrifugal instability(e.g. Brès and Colonius [19]) which depends on the recirculating region in the rear part of cavity.

The adjoint mode $|\hat{\mathbf{v}}|$ shows the region of maximum receptivity to momentum forcing in open cavity flow, as seen in Figure 4.6(c). Note that the adjoint mode, near the upstream lip, is not negligible. The spatial distribution of the product between the direct mode and the adjoint mode $\delta \lambda(x, y, z)$ is displayed in Figure 4.6(d). the results show that "wavemaker" (e.g. Giannetti and Luchini [50]) of the instability is located inside of the cavity. 
4.2. Validation in Nek5000

\begin{tabular}{ccccc}
\hline Runs & & $S t$ & $\lambda_{r}$ & $\lambda_{i}$ \\
\hline \multirow{2}{*}{ DNS } & Brès and Colonius [18] & 0.025 & - & - \\
\cline { 2 - 5 } & icoFoam & 0.02440 & - & - \\
\hline \multirow{2}{*}{ Linear instability } & Brès and Colonius [18] & 0.025 & - & - \\
\cline { 2 - 5 } & dirIcoFoam & 0.02548 & 0.0076 & 0.1601 \\
\hline Adjoint linear instability & adjIcoFoam & 0.02561 & -0.0076 & 0.1609 \\
\hline
\end{tabular}

Table 4.6: Comparison of leading mode of 3D instability results from the open cavity by residual algorithm.

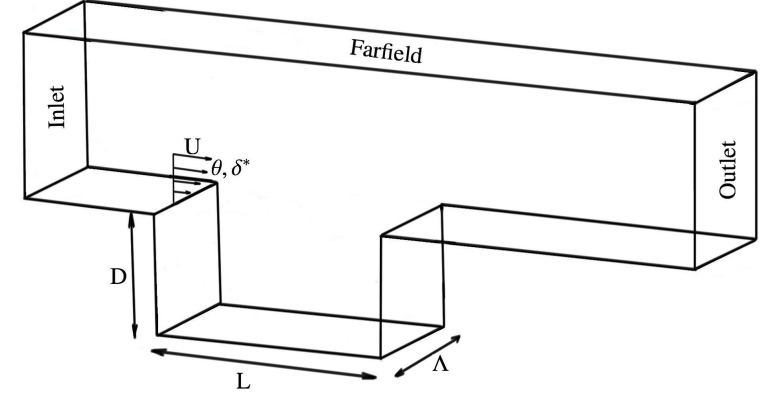

(a) cavity configuration

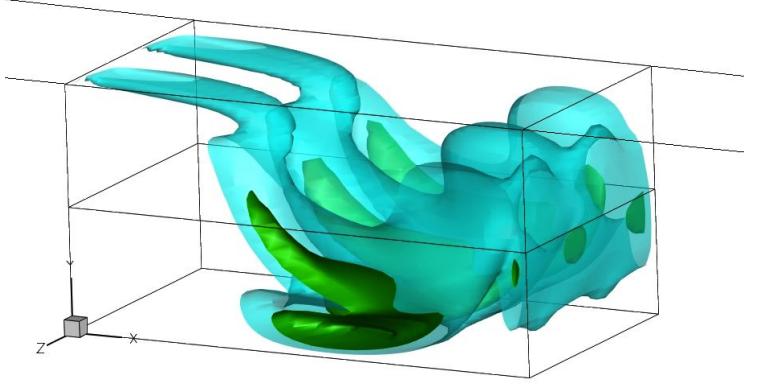

(c) $|\hat{\mathbf{v}}|$

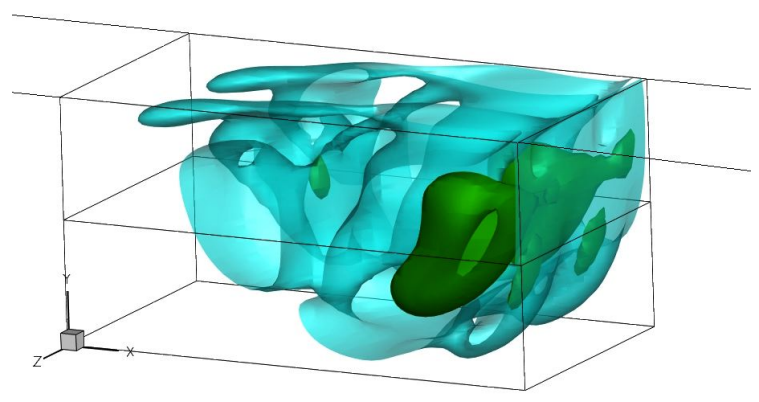

(b) $|\hat{\mathbf{u}}|$

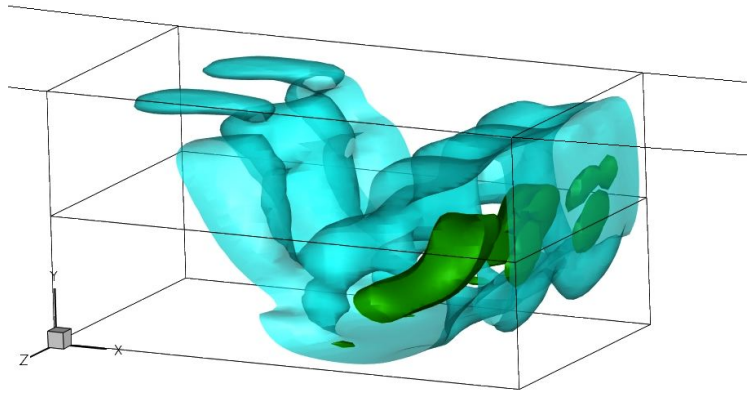

(d) $\delta \lambda(x, y, z)$

Figure 4.6: Normalized module of the $|\hat{\mathbf{u}}|$ direct eigenmode $|\hat{\mathbf{v}}|$ adjoint eigenmode and sensitivity function $\delta \lambda(x, y, z)$ of the open cavity at $R e=1400$. Translucid isosurfaces represent a normalized value of 0.2 ans solid isosurfaces indicate a value 0.5 .

\subsection{Validation in Nek5000}

\subsubsection{Two-dimensional lid-driven cavity validation}

The lid-driven cavity problem is one of the most important benchmarks for numerical Navier-Stokes solvers. The base flow results of the $2 \mathrm{D}$ square lid-driven cavity at $R e=2000$ have served as validation case; a grid convergence analysis has been compared with the benchmark data created by Albensoeder and Kuhlmann [2] and the transient solver quality have tested comparing the damping ratio using the residual algorithm described in Theofilis [112]. 


\begin{tabular}{lccccccccc}
\hline Method & AR & $N_{x}$ & $N_{y}$ & $v_{\min }$ & $x$ & $v_{\max }$ & $x$ & $u_{\min }$ & $y$ \\
\hline SEM & $1: 1$ & 240 & 240 & -0.5262 & 0.9100 & 0.3762 & 0.1580 & -0.3879 & 0.1717 \\
\hline SEM & $1: 1$ & 96 & 96 & -0.5270 & 0.9100 & 0.3769 & 0.1580 & -0.3884 & 0.1717 \\
\hline SCM $^{\dagger}$ & $1: 1$ & 96 & 96 & -0.5270 & 0.9092 & 0.3769 & 0.1578 & -0.3885 & 0.1717 \\
\hline
\end{tabular}

Table 4.7: Comparison of the values of the minimum $u(0.5, y), v(x, 0.5)$ and maximum $v(x, 0.5)$ as well as the position with different resolutions at $R e=1000$ in the lid-driven cavity. $\dagger$ represents the spectral results from Albensoeder and Kuhlmann [2].

\begin{tabular}{cccccccc}
\hline Mesh & elements $\times \mathrm{N}^{2}$ & $v_{\min }$ & $x$ & $v_{\max }$ & $x$ & $u_{\min }$ & $y$ \\
\hline M1 & $144 \times 5^{2}$ & -0.5501 & 0.9300 & 0.4132 & 0.1200 & -0.4193 & 0.1200 \\
\hline M2 & $144 \times 7^{2}$ & -0.5548 & 0.9340 & 0.4140 & 0.1200 & -0.4201 & 0.1200 \\
\hline M3 & $144 \times 9^{2}$ & -0.5558 & 0.9360 & 0.4140 & 0.1200 & -0.4201 & 0.1200 \\
\hline
\end{tabular}

Table 4.8: Comparison of the values of the minimum $u(0.5, y), v(x, 0.5)$ and maximum $v(x, 0.5)$ as well as the position with different resolutions at $R e=2000$ in the lid-driven cavity.

\section{Base flow}

Table 4.7 and table 4.8 show the grid convergence results from the spectral element method: Nek5000[44], the comparison with the research from Albensoeder and Kuhlmann [2] are included. As the resolution is increased, the maximum and minimum velocity components in the center line in the lid-driven cavity are in good agreement with the benchmark date. As shown in figure4.7(a), 12 elements are fixed in the region of $[0,1]$ in $x$ and $y$ direction, respectively, the refined mesh is used near the wall and the inflow direction parallel to $x$ direction and from 0 to 1 . The velocity components $(u, v)$ are plot in figure 4.8, the contour plots are in good agreement with the previous researches.

The base flow is calculated by time-marching until the residual satisfies a pre-determined convergence criterion. Residual algorithm $[114,119]$ is performed on the time history of streamwise velocity in the center of the lid-driven cavity. Figure 4.7(b) shows the damping rate of leading stable global mode. After the initial transient phrase, the numerical solution is governed by a stationary damping mode until convergence attained. The damping rate of this stable mode is calculated $\lambda_{r} \sim-0.068$.

\section{Linear instability analysis results}

In the analysis, a random linear perturbation superimposed upon the base flow used as initial condition, the total integration time $\tau=0.3,10$ eigenvalues have been computed with an initial Krylov basis of dimension 40; the convergence criterion for the Arnoldi iterations is based on a tolerance of $10^{-6}$.

Table 4.9 shows the eigenvalues obtained from current analysis, as well as the eignvalues calculated from different methodologies are listed. All the results are in good agreement. The analysis result confirm the stable flow which comprises both stationary and traveling decaying modes. Additionally, the comparison of the spectrum of eigenvalues from matrixforming and time-stepping are compared in Figure 4.9. The results indicate that the most leading eigenmodes are in good agreement with matrix-forming results.

Figure 4.10(a) and (b) present the two components of the leading global mode, which 


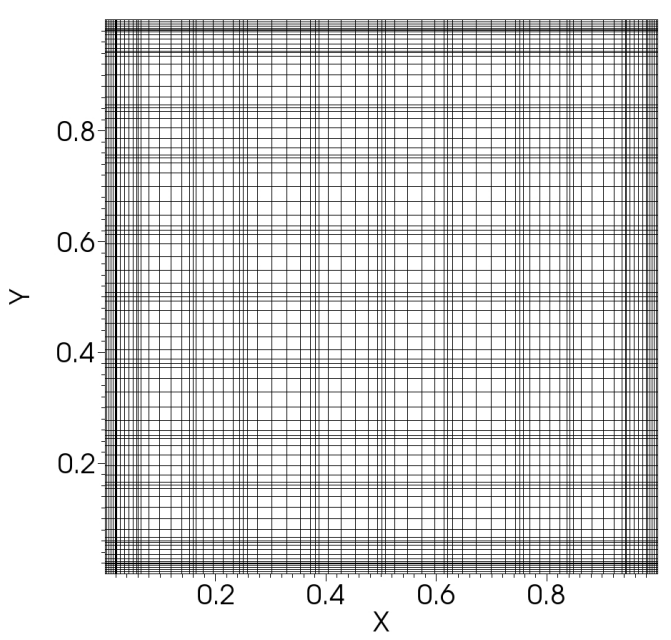

(a)

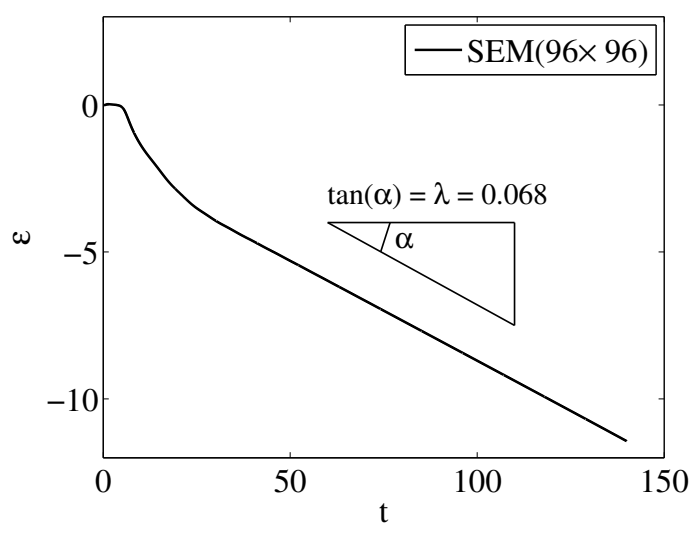

(b)

Figure 4.7: (a) Lid-driven cavity mesh based on the spectral element method. (b) Temporal evolution of the velocity residual of the $2 \mathrm{D}$ lid-driven cavity at $R e=1000$.

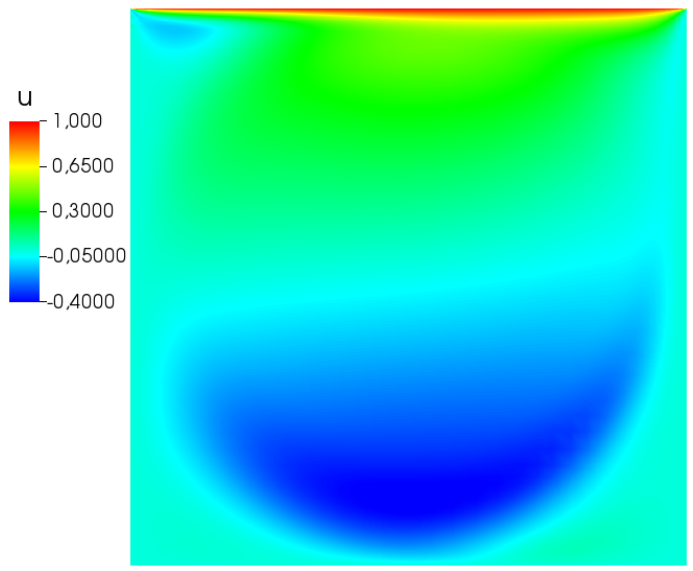

(a) $\mathrm{u}(\mathrm{x}, \mathrm{y})$

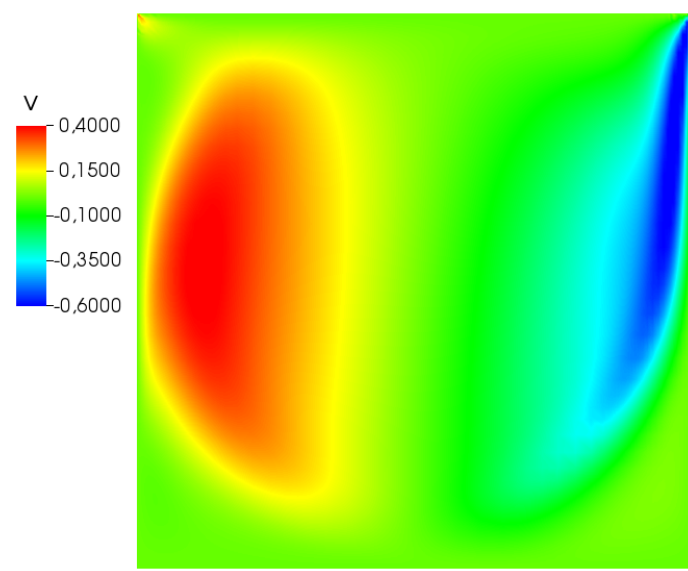

(b) $\mathrm{v}(\mathrm{x}, \mathrm{y})$

Figure 4.8: The steady state stable base flow of lid-driven cavity at $R e=2000$.

\begin{tabular}{ccccc}
\hline & \multicolumn{2}{c}{ Mode I } & \multicolumn{2}{c}{ Mode II } \\
\cline { 2 - 5 } & $\lambda_{r}$ & $\lambda_{i}$ & $\lambda_{r}$ & $\lambda_{i}$ \\
\hline Current & -0.03170 & 0 & 0.98160 & \pm 0.06627 \\
\hline EVP $^{\dagger}$ & -0.03170 & 0 & 0.99662 & \pm 0.06675 \\
\hline TS $^{*}$ & -0.03175 & 0 & 0.96110 & \pm 0.06457 \\
\hline RA $^{*}$ & -0.03170 & 0 & 0.95878 & \pm 0.05998 \\
\hline
\end{tabular}

Table 4.9: Damping rate and frequency of the leading stationary eigenmode and a traveling eigenmode obtained by solution of the global instability eigenvalue problem at $R e=2000{ }^{\dagger}$ refers to the private communication with J.M. Pérez, ${ }^{*}$ is the results from Gómez et al. [53] 


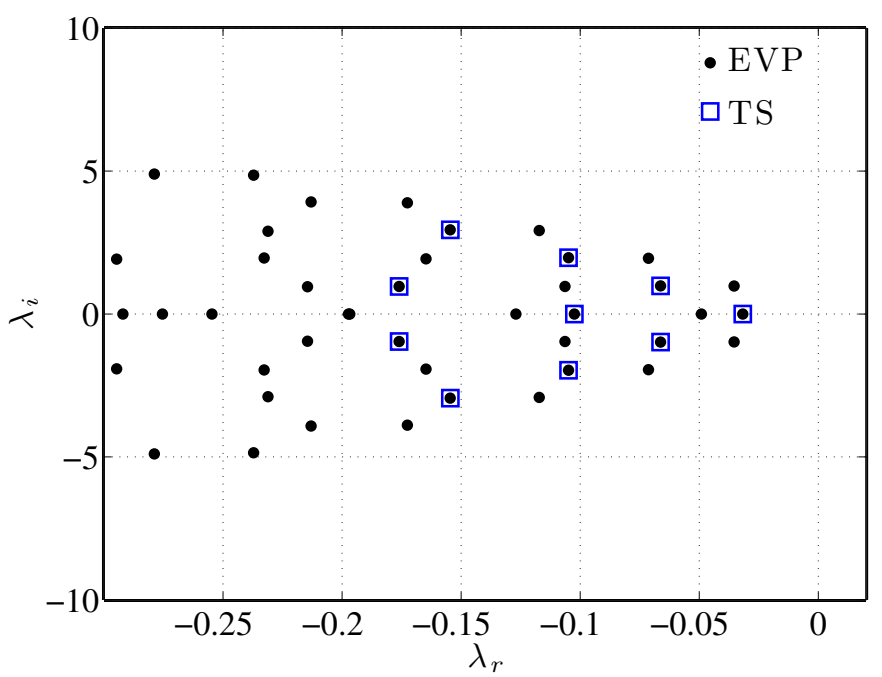

Figure 4.9: The comparison of the spectrum of two-dimensional lid-driven cavity at $R e=2000$ calculated by matrix-forming method (EVP, •) and current method matrix-free method (TS, $\square)$. The matrix-forming results refers to the private communication with J.M. Pérez.

corresponding eigenvalue Mode I in table 4.9, the results are in good agreement with previous from Gómez et al. [53]. Figure 4.10(c) and (d) are flow field of Mode II in table 4.9 . 


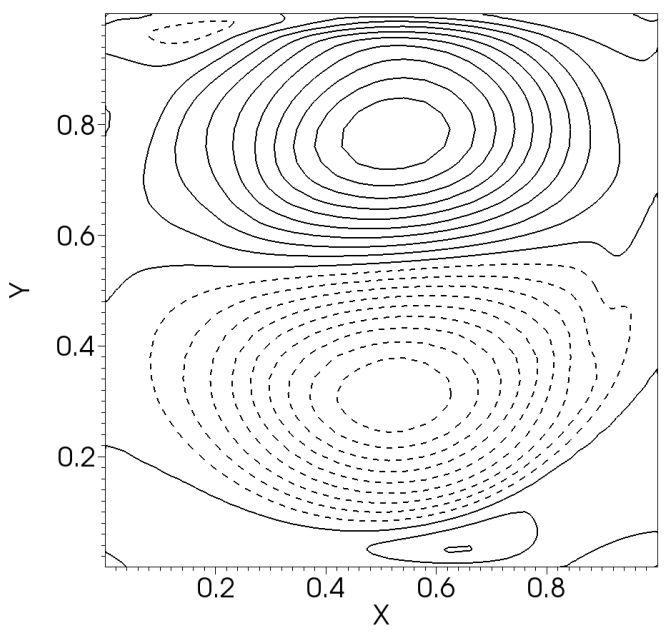

(a) $\hat{u}(x, y)$

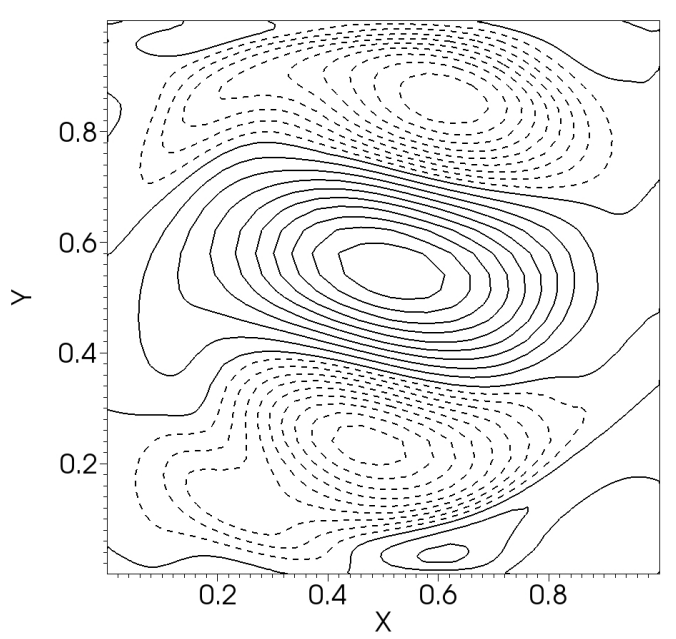

(c) $\hat{u}(x, y)$

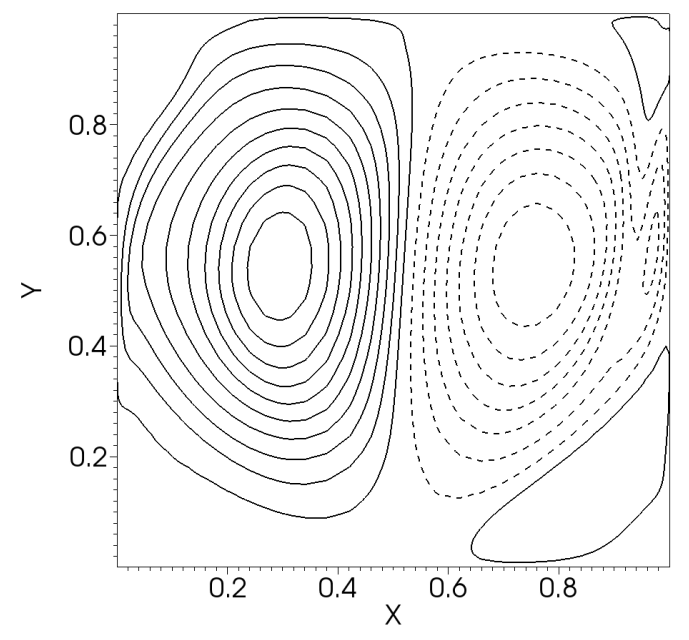

(b) $\hat{v}(x, y)$

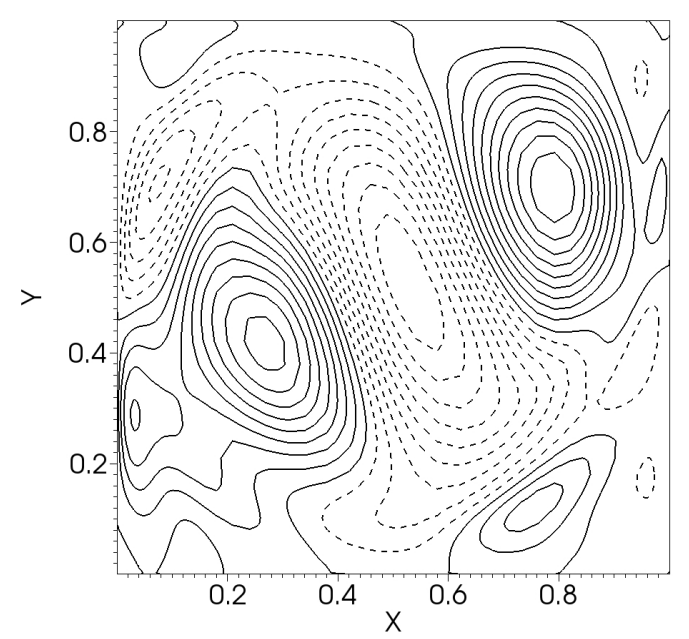

(d) $\hat{v}(x, y)$

Figure 4.10: Eigenmodes of the two-dimensional regularized lid-driven cavity ( LCD) obtained using time-stepping method at $R e=2000$. The solid iso-lines $(-)$ represent positive eigenmodes and the dash iso-line (--) label negative eigenmodes. 10 contour-level of the iso-lines are plotted for the values $\hat{u}(x, y)=[-1,1]$ and $\hat{v}(x, y)=[-0.7,0.65]$, both $\hat{u}(x, y)$ and $\hat{v}(x, y)$ normalized by maximum value of $\hat{u}(x, y)$. 


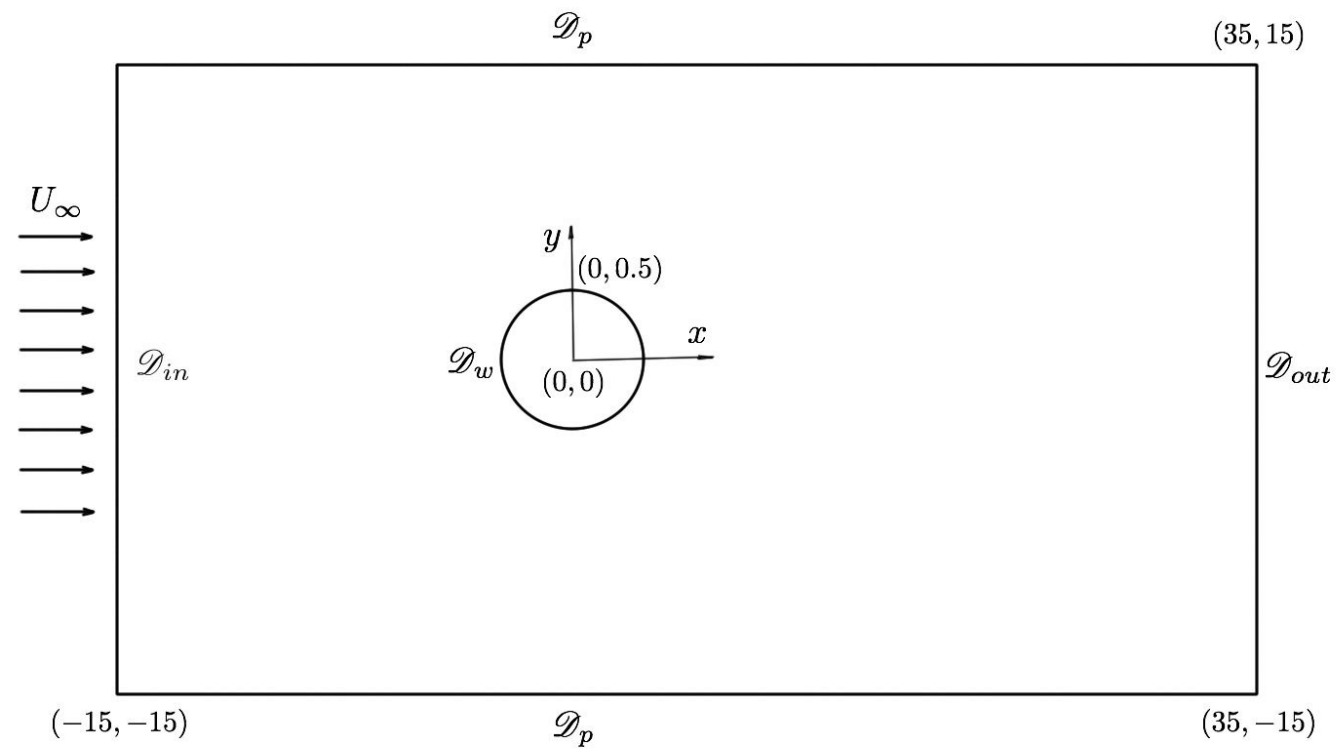

Figure 4.11: Schematic description of the 2D cylinder.

\subsubsection{Two-dimension flow over cylinder}

\section{Base flow}

A cylinder with diameter $D=1$ in a uniform inflow of velocity $U_{\infty}=1$ is proposed as a validation of open flow. The flow quantities are governed by incompressible viscous NavierStokes equations, $R e=U_{\infty} D / \nu$ depended on the diameter of cylinder. The geometry is defined in a Cartesian coordinate system with the original of coordinate $(0,0)$ is in the center of cylinder, the geometry, the frame of reference and the notation adopted in this work are all plotted in figure 4.11. A no-slip boundary condition $U=(u, v)=(0,0)$ is apply on $\mathscr{D}_{w}$, Dirichlet boundary conditions $U=(u, v)=(1,0)$ on $\mathscr{D}_{i n}$, Neumann boundary condition $\partial u / \partial x=0$ on $\mathscr{D}_{\text {out }}$, and periodic boundary conditions on $\mathscr{D}_{p}$.

The numerical procedure described in the preceding section is used to evaluate the steady flow and to carry out the stability analysis. Depend on the grid independent results, simulations of cylinder are performed on the domain $[-15: 35] \times[-15: 15]$ with 34,65 elements in the $x$ and $y$ direction respectively, the polynomial order of Guass Lobatto equations is 7 , which means in every element has $8 \times 8$ points.

The cylinder flow is known to become unstable at a critical Reynolds number $R e_{c} \approx$ $46.7[35,37,49,65,106]$ by computation and $R e_{c} \approx 47$ in the experiments [81, 92, 121].

Figure 4.13 visualizes the spatial variations of $u$ and $v$ as $R e$ increases. A symmetric flow structure can be observed in steady stable state at $R e=40$ in figure 4.13 (a) and (b). When increasing $R e=50$ above the threshold $R e=47$, a strong wake shedding occurs after the cylinder, both flow fields lose the symmetry, as shown in figure 4.13(c) and (d). When $R e=100$, a higher-frequency oscillation appears, with a unstable state in figure 4.13(e) and (f). Steady unstable solution of flow over a cylinder at $R e=100$ are shown in figure $4.13(\mathrm{~g})$ and $(\mathrm{h})$, which is considered as the base flow of linear analysis. 


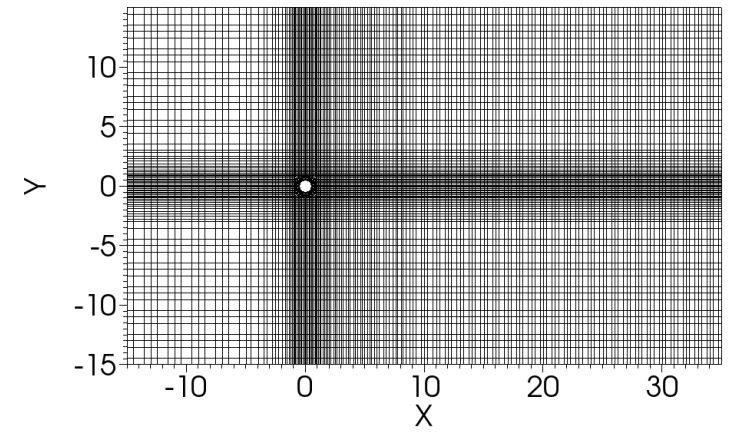

(a)

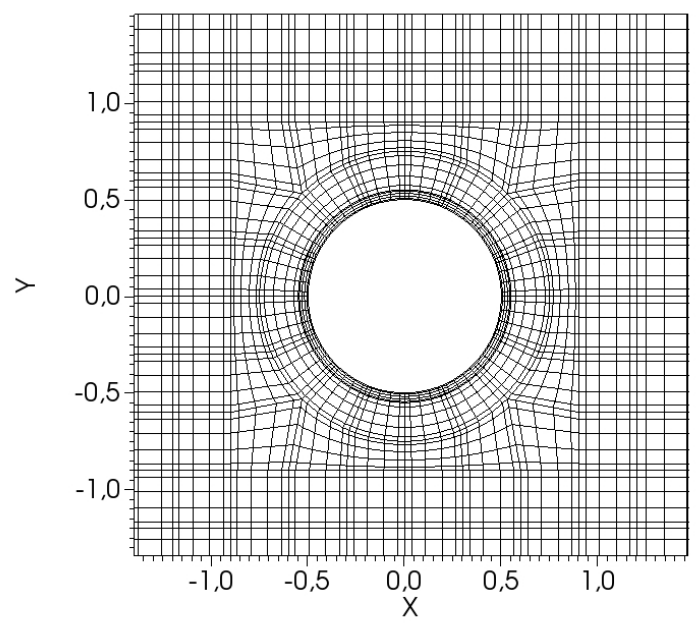

(b)

Figure 4.12: (a) mesh of the cylinder; (b) mesh detail near the cylinder . 


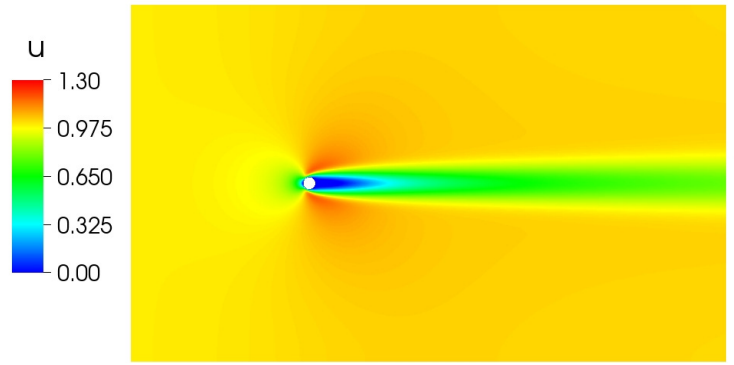

(a) $u(x, y)$

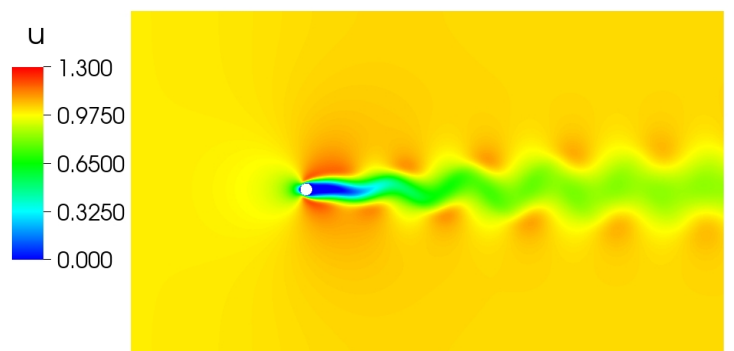

(c) $u(x, y)$

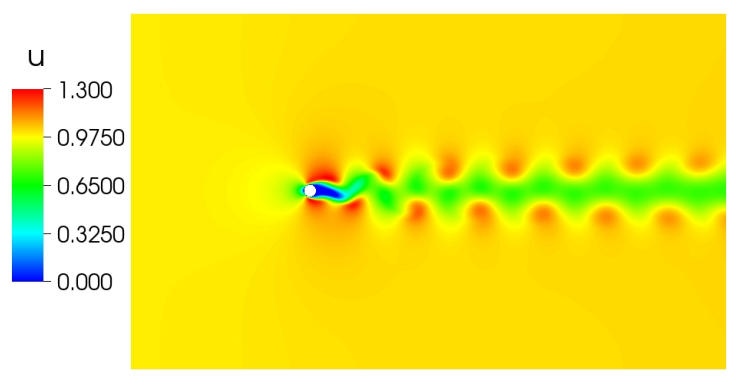

(e) $u(x, y)$

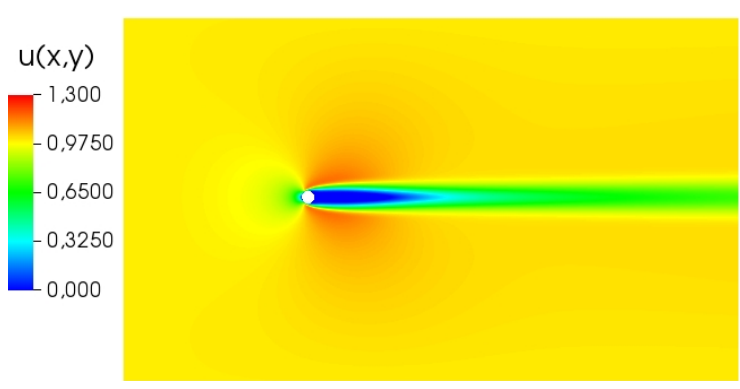

(g) $\bar{u}(x, y)$

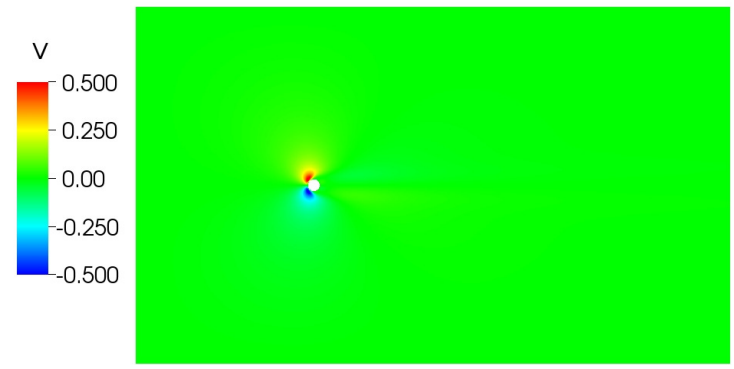

(b) $v(x, y)$

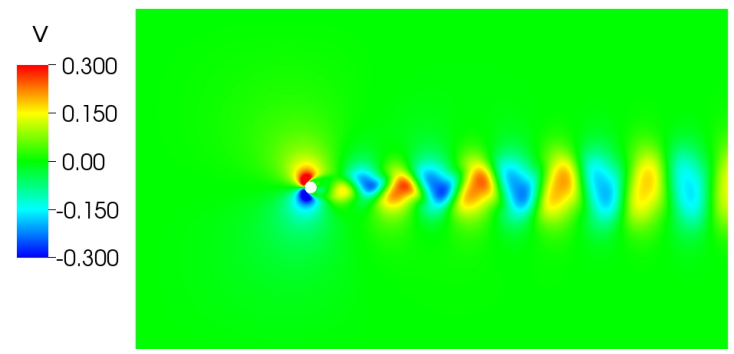

(d) $v(x, y)$

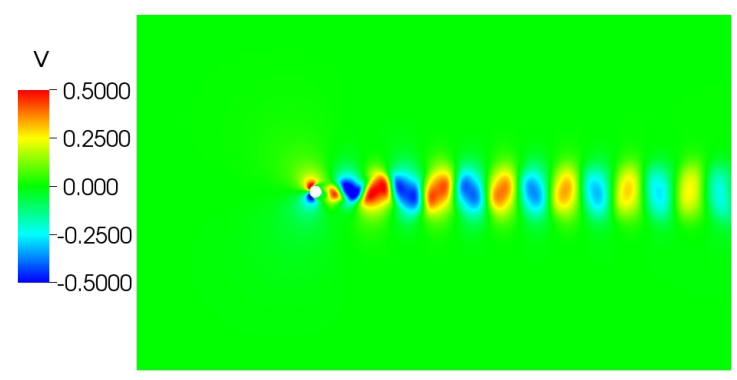

(f) $v(x, y)$

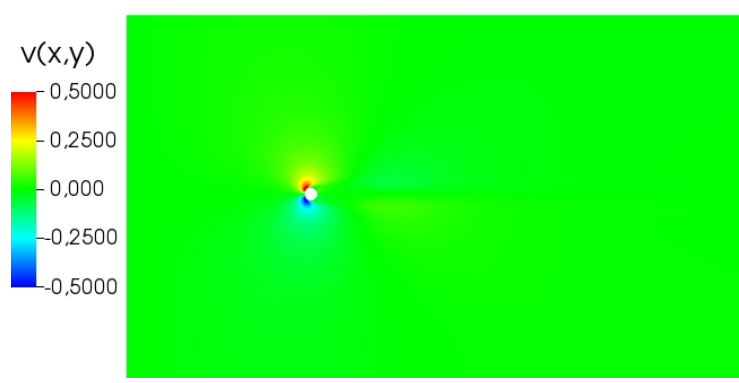

(h) $\bar{v}(x, y)$

Figure 4.13: The snapshot of the time-periodic solution of (a) $u(x, y)$ and (b) $v(x, y)$ at $R e=40$; The snapshot of the time-periodic solution of $(\mathrm{c}) u(x, y)$ and $(\mathrm{d}) v(x, y)$ at $R e=50$; The snapshot of the time-periodic solution of (e) $u(x, y)$ and (f) $v(x, y)$ at $R e=100$; The steady unstable base flow $(\mathrm{g}) u(x, y)(\mathrm{h}) v(x, y)$ of $R e=100$ from selective frequency damping method. 


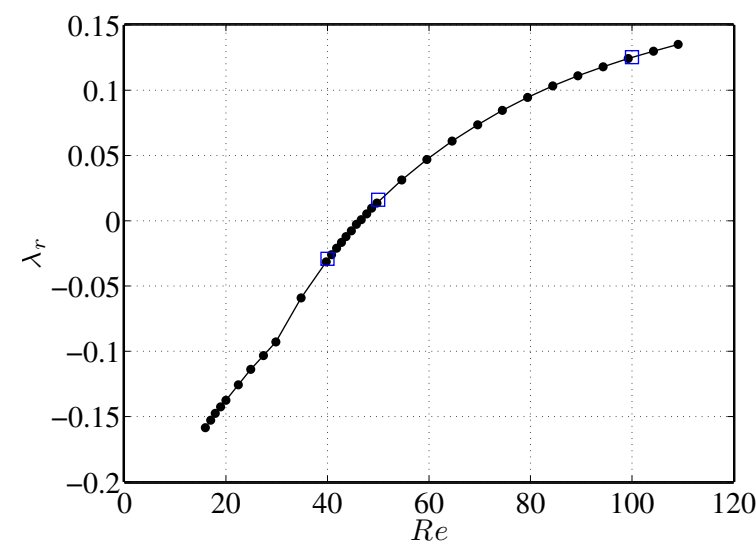

(a)

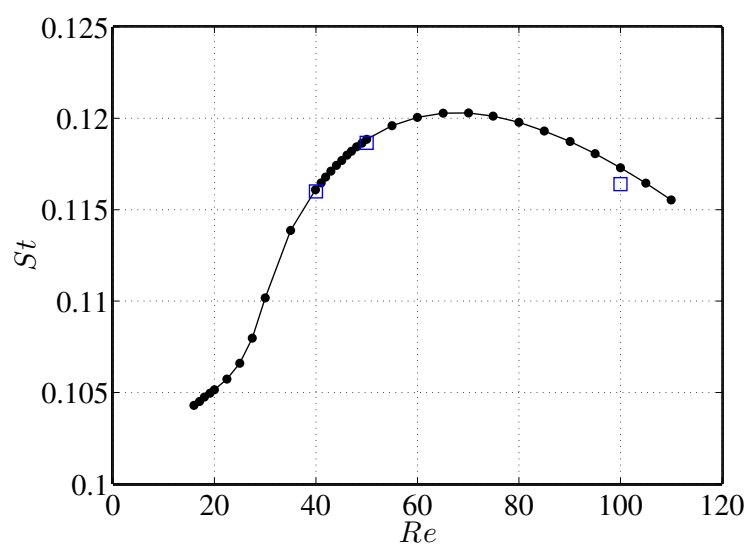

(b)

Figure 4.14: (a) Amplification rate and (b) Strouhal number dependence on Re from the previous research of Giannetti and Luchini [49], $\square$ represent the results from current research.

\section{Linear instability analysis}

The stability characteristics of the base flow are assessed by monitoring the behavior of the most unstable mode in the linearized systems. Figure 4.14 shows the amplification rate $\lambda_{r}$ and the Strouhal number $S t=\lambda_{i} / 2 \pi$ of the leading global mode, where $\lambda_{i}$ is the circular frequency. According to current calculations, the eigenvalues of cylinder at $R e=40$, $R e=50$ and $R e=100$ are in good agreement with the numerical results of Giannetti and Luchini [49], as shown in figure 4.14(a) and 4.14(b). In this time-stepping analysis, Krylov subspace dimensions is $m=40$ for calculating 10 eigenvalues, the tolerance of the Arnoldi iteration is $10^{-6}$, while time integration varies as $R e$ changes, time integration is $\tau=0.3$ at $R e=40, \tau=0.4$ at $R e=50$, and $\tau=1$ at $R e=100$. The leading eigenmode is depicted in figure 4.15 of different Reynolds number by the real part of its velocity field, the modes $\hat{u}(x, y)$ are antisymmetric, as the Reynolds number increased, the maximum magnitude of unstable mode spatially towards to the cylinder. 


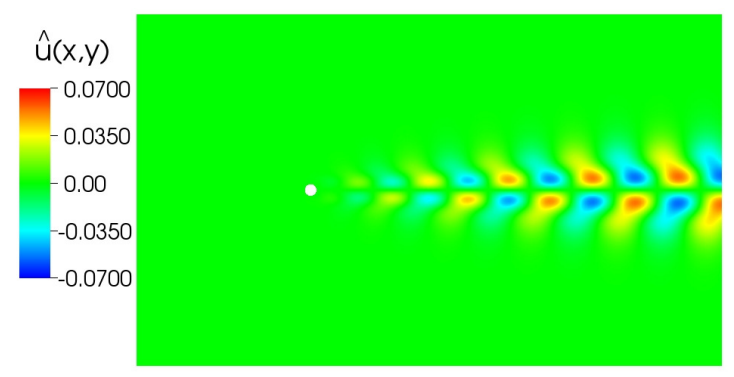

(a) $\hat{u}(x, y)$

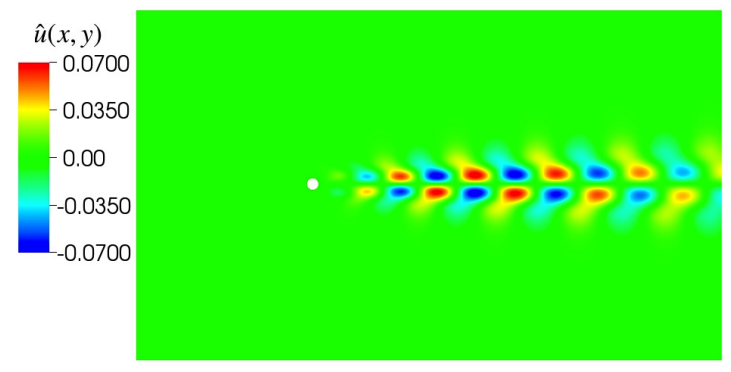

(c) $\hat{u}(x, y)$

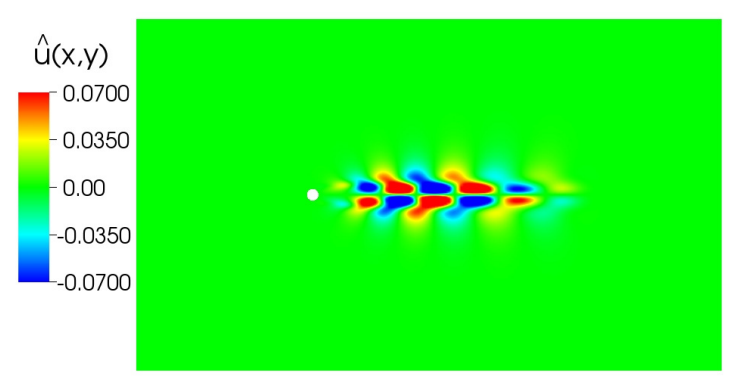

(e) $\hat{u}(x, y)$

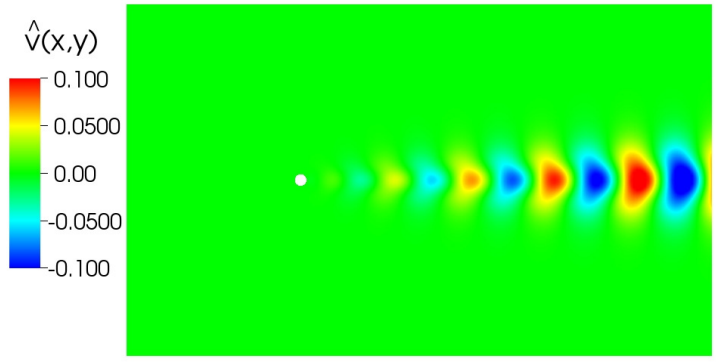

(b) $\hat{v}(x, y)$

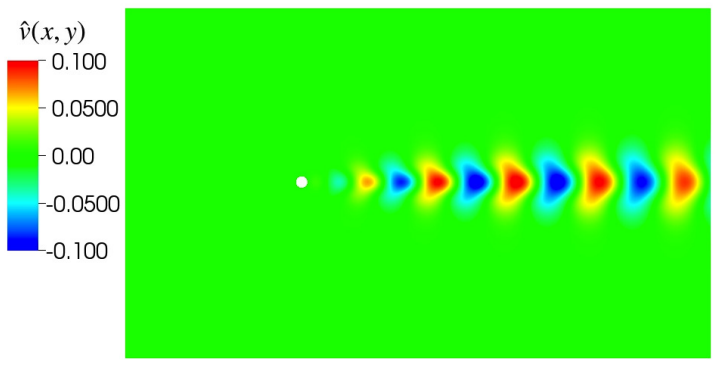

(d) $\hat{v}(x, y)$

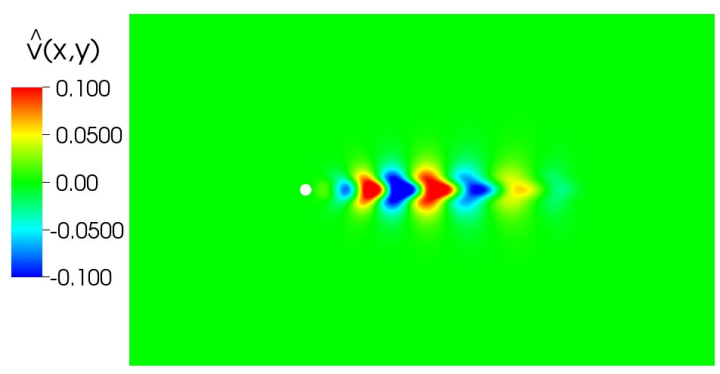

(f) $\hat{v}(x, y)$

Figure 4.15: (a), (b) The leading unstable eigenmode $\lambda=-0.0291+i \cdot 0.7485$ of cylinder at $R e=40$. (c), (d) The leading unstable eigenmode $\lambda=0.01611+i \cdot 0.7452$ of cylinder at $R e=50$. (e), (f) The leading unstable eigenmode $\lambda=0.1253+i \cdot 0.7310$ of cylinder at $R e=100$ 


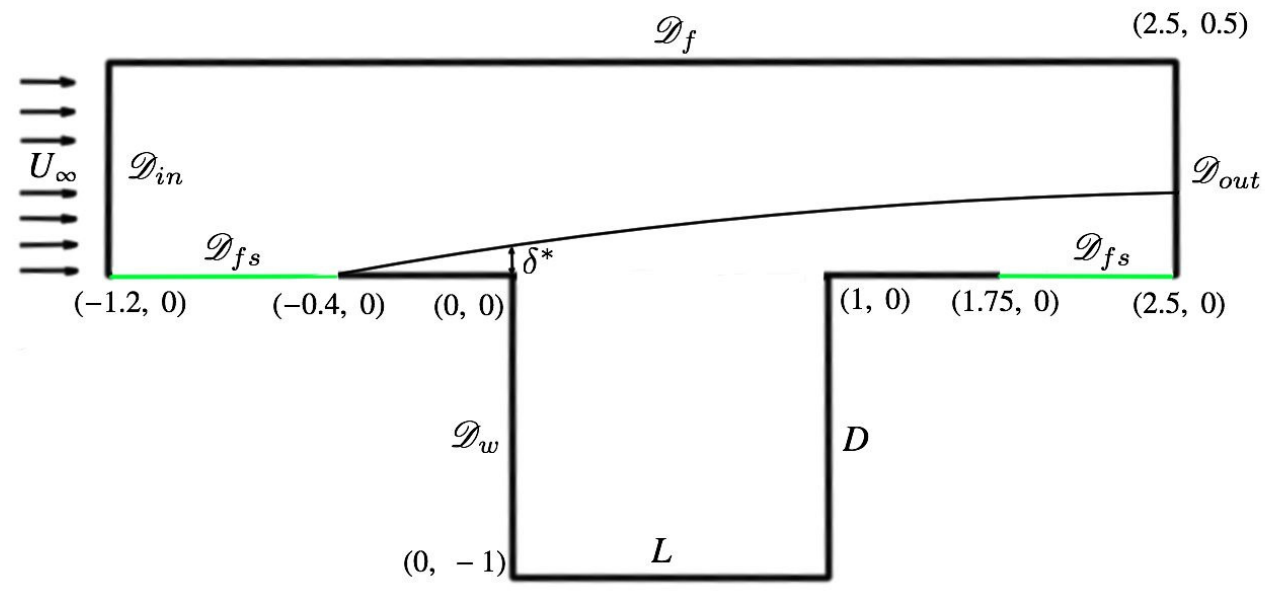

Figure 4.16: Schematic description of the square open cavity.

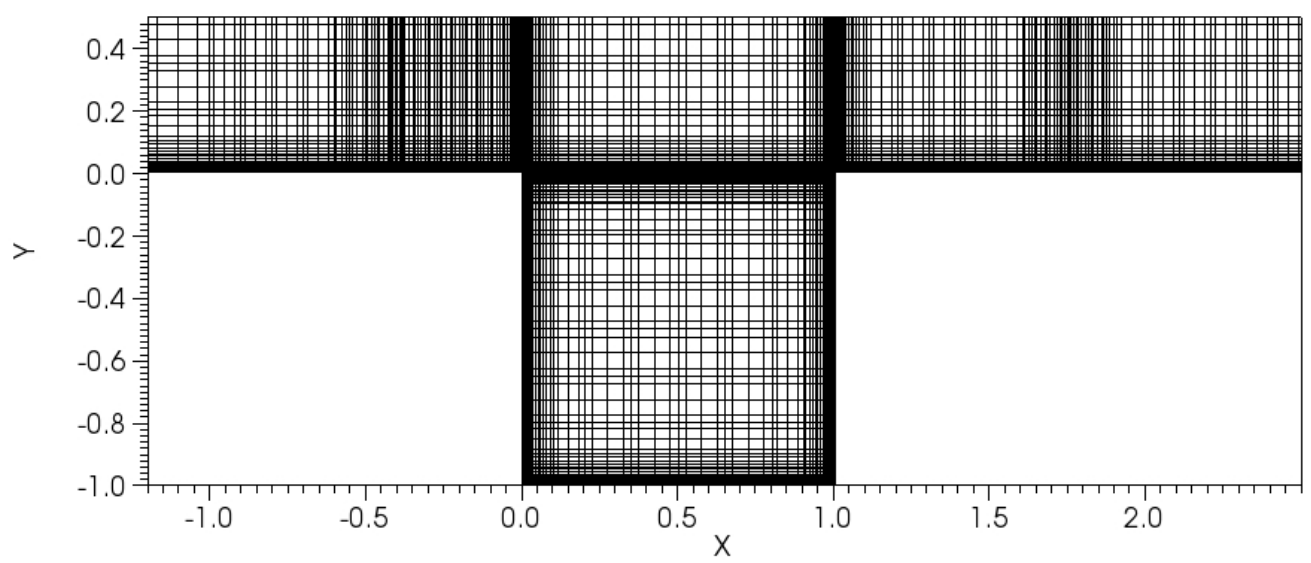

Figure 4.17: Mesh information of the square open cavity.

\subsubsection{Square open cavity with $L: \Lambda: D=1: 1: 1$}

\section{Base flow}

Direct numerical simulation of the flow over a spanwise-periodic square open cavity has been investigated. The computational geometry and the notation of boundary condition adopted in this work are all displayed in figure 4.16. The original of the Cartesian reference system is located on the upstream of the cavity with $x, y$ and $z$ denoting the streamwise, transverse (wall-normal) and spanwise directions. The cavity depth $D=1$ as the characteristic length scale and the length to depth ratio is $L / D=1$, the uniform incoming velocity $U_{\infty}=(u, v)=(1,0)$ as the reference velocity, the Reynolds number is $R e=U_{\infty} D / \nu=4140$ which is larger than the critical Reynolds number of square open cavity $R e_{\text {crit }} \approx 1370$ [27].

The mesh is generated by the spectral element method (genbox) that is part of the Nek5000 package. The refined mesh is supplied in the boundary layer and near the wall in 


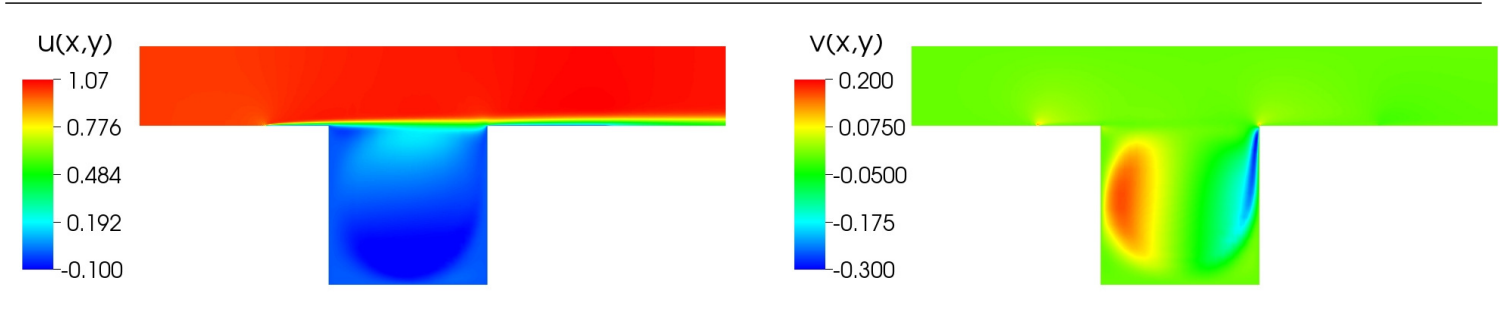

(a) $u(x, y)$

(b) $v(x, y)$

Figure 4.18: (a), (b) 2D steady unstable base flow of square open cavity ( $L: D: \Lambda=1: 1: 1)$ flow at $R e=4140$.

\begin{tabular}{rc}
\hline Domain & Elements \\
\hline$[-1.2,-0.4] \times[0,0.5]$ & $10 \times 7$ \\
\hline$[-0.4,0] \times[0,0.5]$ & $12 \times 7$ \\
\hline$[0,1] \times[0,0.5]$ & $14 \times 7$ \\
\hline$[1,1.75] \times[0,0.5]$ & $13 \times 7$ \\
\hline$[1.75,2.5] \times[0,0.5]$ & $10 \times 7$ \\
\hline
\end{tabular}

Table 4.10: Mesh details of the 2D square open cavity.

order to make sure accurately spatial analog, as shown in figure $4.17 .14 \times 14$ elements are set in the square cavity. The length of first elements which attaches the wall and shear layer are fixed to 0.015 , it is approximate to the displacement thickness $\delta^{*}=0.0169$ of the boundary layer. 5 domains are defined outside of the cavity.

The mesh information are documented in table 4.10. It is noteworthy that because of the change of boundary condition in the upstream and downstream of the cavity, the refined mesh are needed near the intersection.

To ease comparison, the computational domain is the same to Citro et al. [27], as well as the same boundary conditions in Sipp and Lebedev [107], Barbagallo et al. [9] and Citro et al. [27]: The Dirichlet boundary conditions at the inflow $\mathscr{D}_{\text {in }}$ and the stress-free conditions at the outlet $\mathscr{D}_{\text {out }}$, symmetric condition (i.e. $\partial_{y} u=0$ and $v=0$ ) are imposed at the free-stream upper boundary of the computational domain $\mathscr{D}_{f}$ and no-slip conditions $(U=0)$ at the solid walls $\mathscr{D}_{w}$, Note that a slip condition (i.e. $\partial_{y} u=0$ and $\left.v=0\right)$ with zero tangential stress is used on the walls close to the upstream $\mathscr{D}_{f s}$ and downstream $\mathscr{D}_{f s}$. See in figure 4.16 .

The steady base flow of the $2 \mathrm{D}$ square open cavity at $R e=4140$ is calculated from steady version incompressible Navier-Stokes equations by the selective frequency damping method[1], the process is continued until the $L^{2}$-norm of the residual of the governing equations becomes smaller than $10^{-12}$.

Figure 4.18 presents the two components of steady unstable base flow. This base flow is resemble to the flow over lid-driven cavity.

\section{Linear instability analysis}

BiGlobal Stability analysis is carried out on the two-dimensional steady unstable base flow with a third homogeneous direction, the periodic spanwise wavelength is $\Lambda=0.285$. 


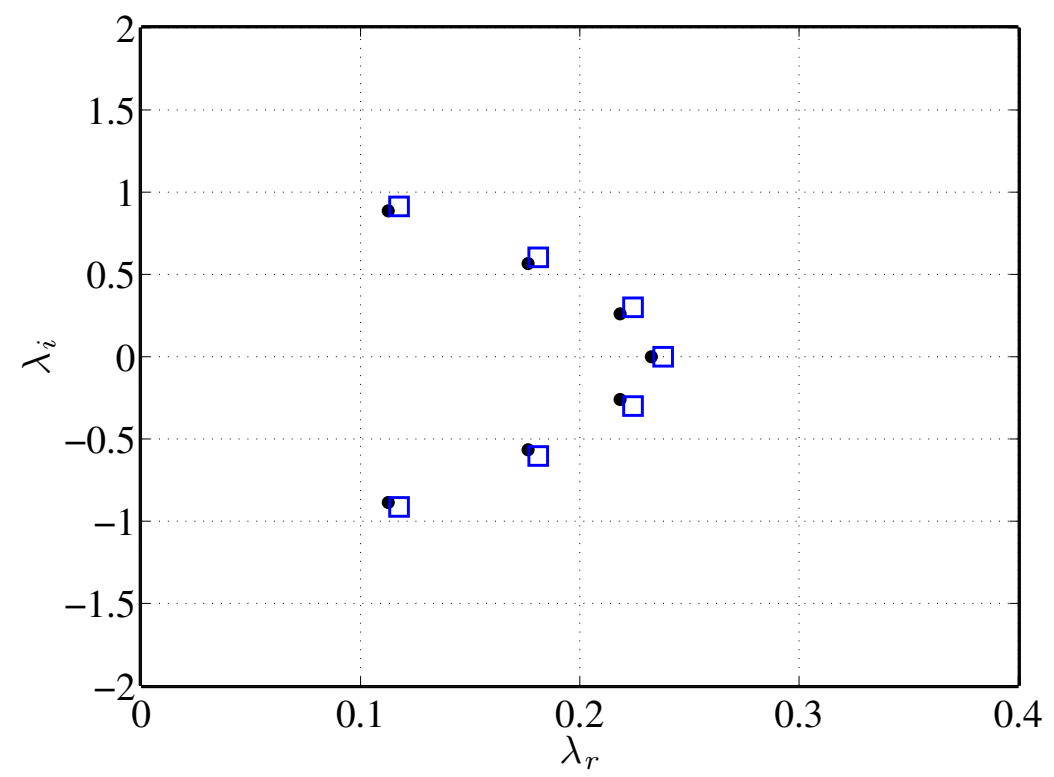

Figure 4.19: Comparison of eigenvalue spectrum of square open cavity at $R e=4140$ and $\beta=22$ from current results $(\square)$ and results from Citro et al. [27] $(\bullet)$.

\begin{tabular}{cc}
\hline Mode & $\lambda_{r} \pm \lambda_{i}$ \\
\hline I & $0.2380 \pm \mathrm{i} \cdot 0.0000$ \\
\hline II & $0.2243 \pm \mathrm{i} \cdot 0.3005$ \\
\hline III & $0.1811 \pm \mathrm{i} \cdot 0.6036$ \\
\hline IV & $0.1176 \pm \mathrm{i} \cdot 0.9134$ \\
\hline
\end{tabular}

Table 4.11: Unstable eigenvalues of the square open cavity at $R e=4140$ and $\beta=22$.

Seven unstable global modes are obtained using time-stepping method. The growth rate and frequency of these unstable modes are compared with the results of Citro et al. [27], as shown in figure 4.19, they are in good agreement. Table 4.11 presents the value of growth rate and frequency. It is obviously to know the most unstable mode is steady, while the others have low frequencies.

By review perturbation flow field, it is clear that the external flow play little role of the three-dimensional instability in this square open cavity geometry. The velocity perturbations are most evident insider of the cavity, with a tail in the shear region just around and above the downstream, see in the figure 4.28. This three-dimensional instability, named centrifugal instability, is related to the primary vortex inside cavity. It is quit similar to the instability property of lid-driven cavity flow [107? ? ? ? ? ]. 


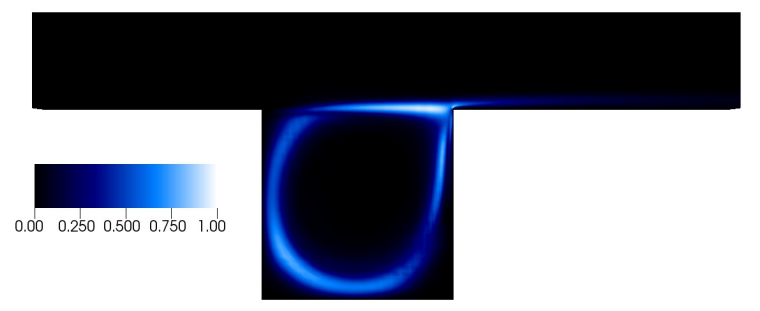

(a) $|\hat{\boldsymbol{u}}(x, y, z)|$

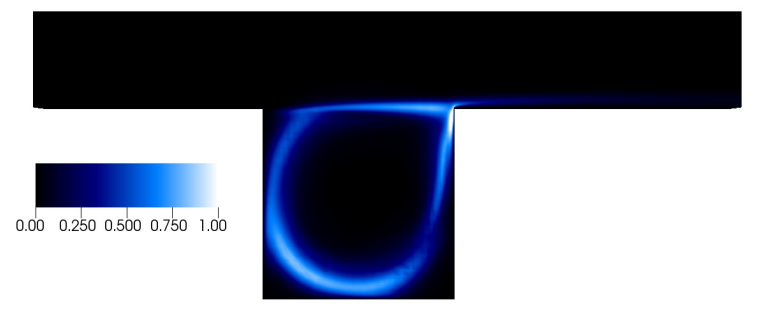

(c) $|\hat{\boldsymbol{u}}(x, y, z)|$

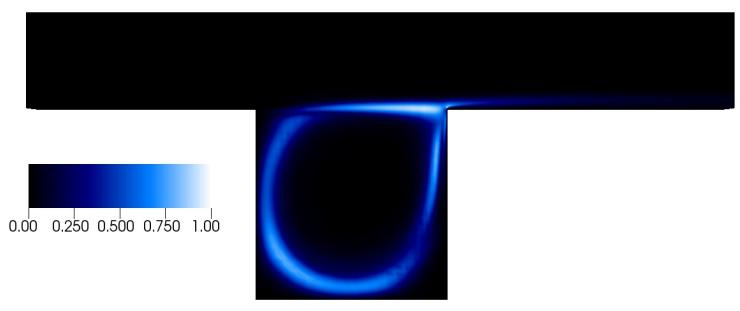

(b) $|\hat{\boldsymbol{u}}(x, y, z)|$

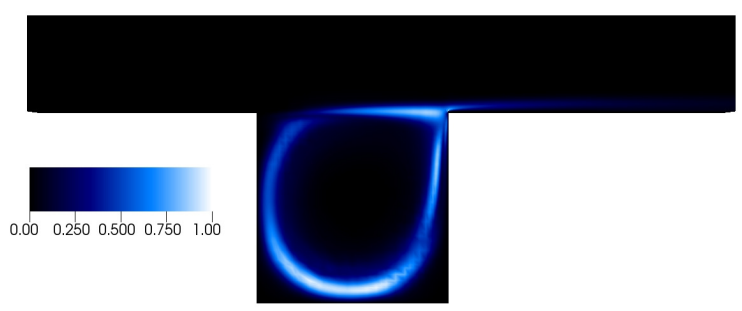

(d) $|\hat{\boldsymbol{u}}(x, y, z)|$

Figure 4.20: Contour plots of the direct modes: (a) mode I: $0.2380+i \cdot 0.000$, (b)mode II $: 0.2243+i \cdot 0.3005,(\mathrm{c})$ mode III: $0.1811+i \cdot 0.6036$, (d) mode IV: $0.1176+i \cdot 0.9134$. 


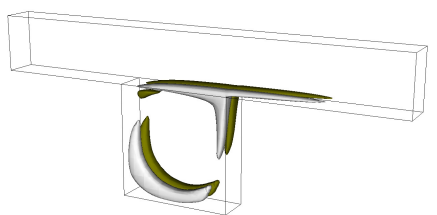

(a) $\hat{u}(x, y, z)$

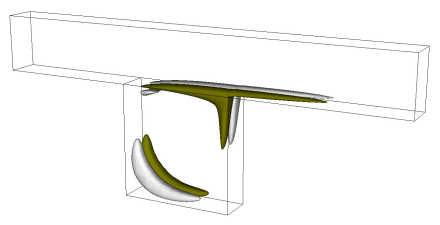

(d) $\hat{u}(x, y, z)$

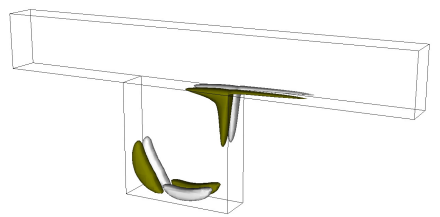

(g) $\hat{u}(x, y, z)$

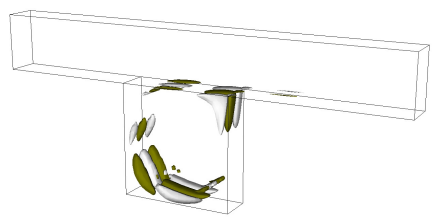

(j) $\hat{u}(x, y, z)$

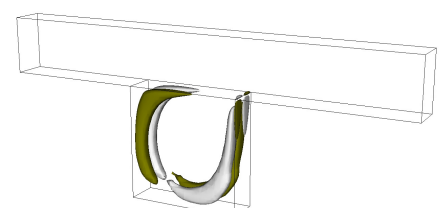

(b) $\hat{v}(x, y, z)$

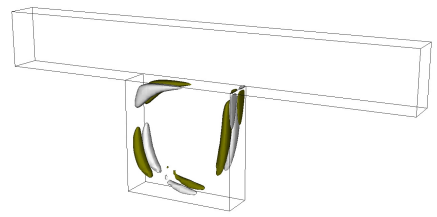

(e) $\hat{v}(x, y, z)$

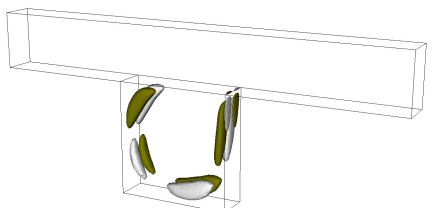

(h) $\hat{v}(x, y, z)$

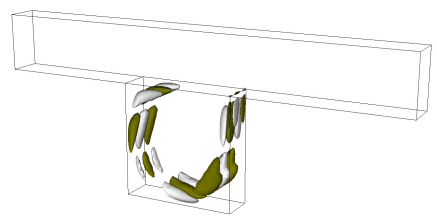

(k) $\hat{v}(x, y, z)$

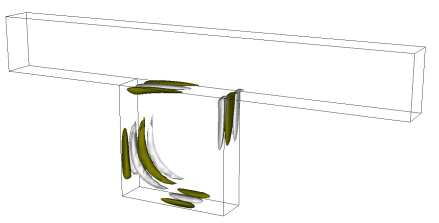

(c) $\hat{w}(x, y, z)$

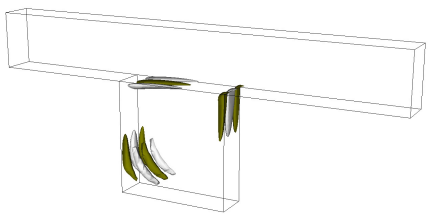

(f) $\hat{w}(x, y, z)$

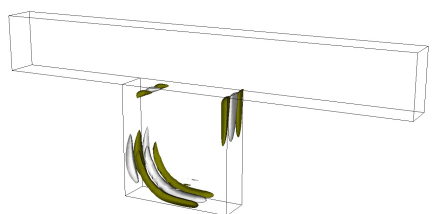

(i) $\hat{w}(x, y, z)$

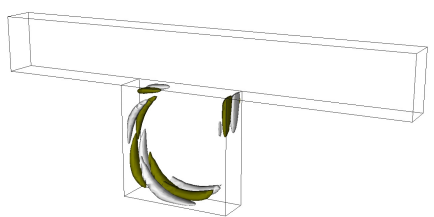

(l) $\hat{w}(x, y, z)$

Figure 4.21: Real part of the eigenfunction velocity field of the spanwise periodic square open cavity at $R e=4140$. Eigenfunctions normalized $\max (\hat{u})$. (a)-(c)mode I: $0.2380+i \cdot 0.000$; (d)(f)mode II :0.2243 $+i \cdot 0.3005$; (g)-(i)mode III: $0.1811+i \cdot 0.6036$; (g)-(i)mode IV: $0.1176+i$. 0.9134. The iso-surface level are: $\hat{v}(x, y, z), \hat{v}(x, y, z), \hat{w}(x, y, z)= \pm 0.15$. 


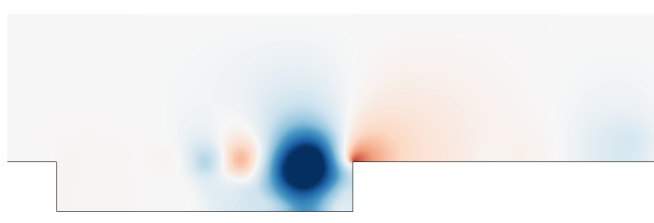

(a) $p$

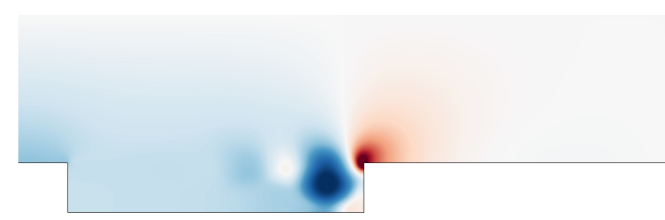

(c) $p$

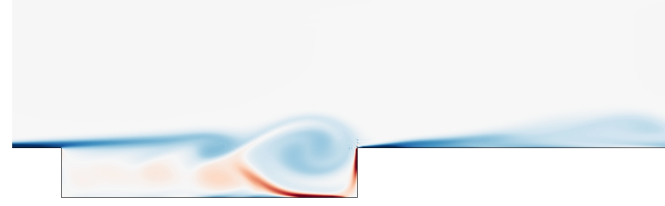

(b) $\omega_{z}$

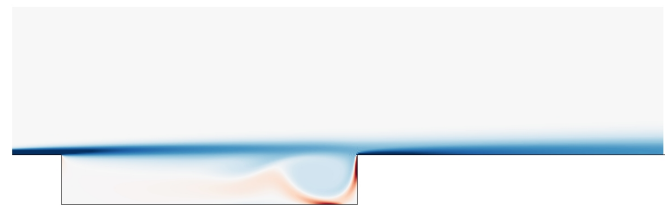

(d) $\omega_{z}$

Figure 4.22: The instantaneous numerical schilieren of (a) $p= \pm 0.4$ and (b) $\omega_{z}= \pm 8$ from DNS. The steady state of (c) $p= \pm 0.04$ and (b) vorticity contours $\omega_{z}= \pm 5$ using SFD.

\subsubsection{Spanwise Periodic Open Cavity $L: \Lambda: D=6: 2: 1$}

\section{Base flow}

The spanwise periodic three-dimensional open cavity of the aspect ration $L / D=$ $6, \Lambda / D=2$ is studied here, which $\Lambda$ is the spanwise wavelength of homogeneous direction. DNS is performed on two-dimensional open cavity $L / D=6$ at $R e=1000$. The flow has been proven to be unstable by reviewing the study of Sun et al. [110], the critical Reynolds number of this kind of open cavity flow has been reveal in the range of $700<R e_{c r}<800$. The selective frequency damping method[1] (SFD) are used to get the steady unstable base flow

Figure 4.22 illustrates the pressure and vorticity contours from DNS and SFD. Initially, the shear layer instability is observed in the unstable results, as shown in figure 4.22(b), the vortex with the longitude about $2 L / 5$ rolls up in the rear part of the cavity, the contour plot levels are \pm 0.4 , that is 10 times of the steady-state case, the recirculation zone inside of the cavity break up into several vortices because of the interrelationship. In the figure $4.22(\mathrm{~d})$, the shear layer keeps horizontal along the cavity, a large recirculation zone preserves over the extent of the cavity, the contour levels of vorticity are \pm 0.04 , even the shear layer vortex and primary vortex have a opposite sign, both are too weak to interfere each other.

\section{Linear Instability Analysis}

Linear instability analysis is carried on the obtained steady unstable two-dimensional base flow imposing a homogeneous spanwise wavenumber $\beta=\pi$, the parameters employed in the time-stepping method are chosen: Krylov subspace dimension $m=20$, time integration $\tau=2,0.48$ and the tolerance of the Arnoldi iteration $10^{-6}$.

The first three unsteady modes acquired, as shown in the table 4.12. The first and secondary unstable mode are related to the Rossiter mode II $(\sim 1.0)$ and Rossiter mode III $(\sim 1.5)$, The Strouhal number of the third mode is the typical centrifugal instability 


\begin{tabular}{ccc}
\hline Parameters & $\tau=2$ & $\tau=0.48$ \\
\hline Mode & $\lambda_{r} \pm i \cdot \lambda_{i}$ & $\lambda_{r} \pm i \cdot \lambda_{i}$ \\
\hline 1 & $0.0850 \pm i \cdot 1.0537$ & $0.0850 \pm i \cdot 1.0537$ \\
\hline 2 & $0.0798 \pm i \cdot 1.5313$ & $0.0798 \pm i \cdot 1.5313$ \\
\hline 3 & $0.0320 \pm i \cdot 0.2427$ & $0.0320 \pm i \cdot 0.2427$ \\
\hline
\end{tabular}

Table 4.12: The first three leading unstable eigenvalues of the spanwise periodic open cavity at the $R e=1000$.

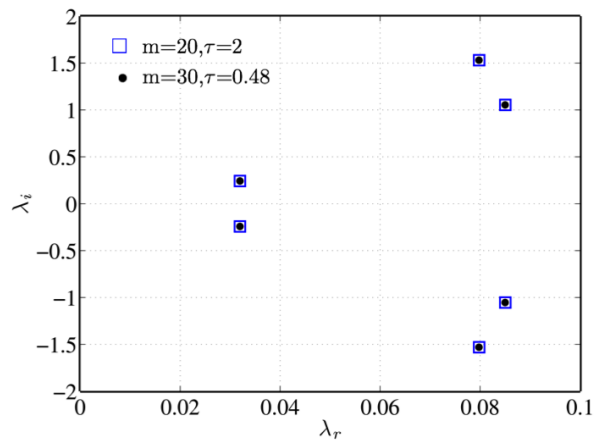

Figure 4.23: Spectrum of spenwise periodic open cavity L: $\Lambda: D=6: 2: 1$ at $R e=1000$.

frequency $(0.02 \sim 0.03)$.

In figure 4.24, spatial structure of the three unstable eigenfunctions are summarized. The Mode 1 and Mode 2 corresponds Rossiter mode II and Rossiter III, respectively, both unstable mode exhibit two-dimensionality property, without spanwise distribution. Mode 3 is three-dimensional centrifugal mode, the eigenfunction locates inside of the cavity. This global mode associates with the recirculation region in the rear part of the cavity. 


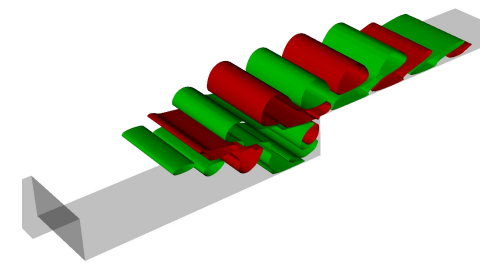

(a) $\hat{u}(x, y, z)$

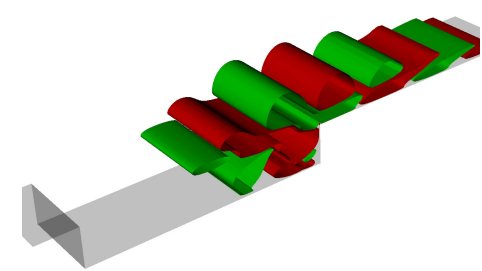

(c) $\hat{u}(x, y, z)$

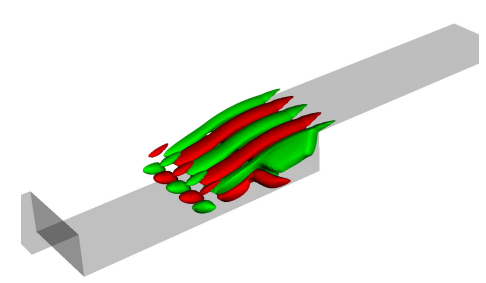

(e) $\hat{u}(x, y, z)$

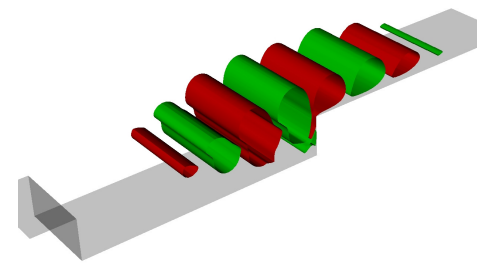

(b) $\hat{v}(x, y, z)$

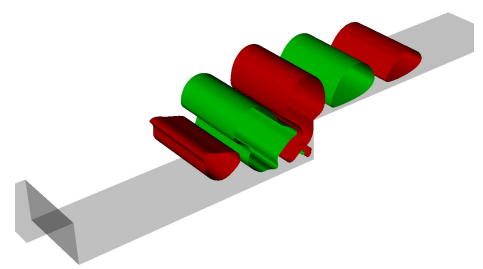

(d) $\hat{v}(x, y, z)$

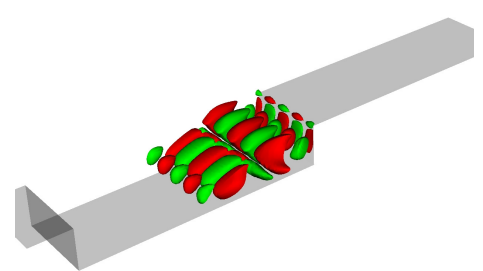

(f) $\hat{v}(x, y, z)$

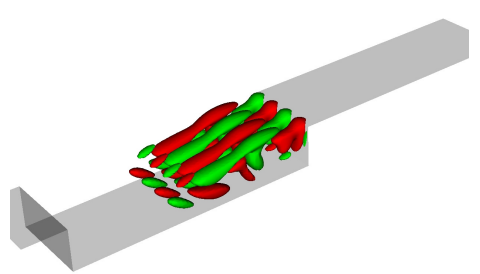

(g) $\hat{w}(x, y, z)$

Figure 4.24: Real part of the eigenfunctions velocity field of the $3 \mathrm{D}$ cavity at $R e=1000$, eigenfunctions normalized with $\max (\hat{u})$. 


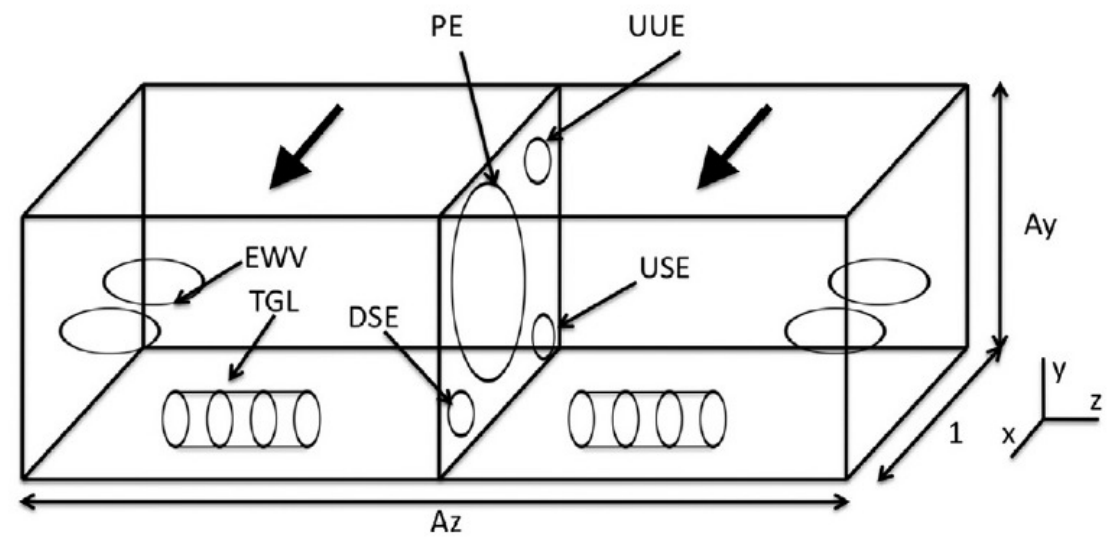

Figure 4.25: Geometrical description of the problem and main flow features from Gómez et al. [54]

\subsubsection{Three-dimensional lid-driven cavity validation}

\section{Base flow}

The instability of the three-dimensional lid-driven cubic cavity has received some significant advances, Giannetti et al. [51] found that a stationary mode becomes unstable just above $R e \sim 2000$ by means of the first TriGlobal linear stability analysis applied to this kind of flows. Late, Liberzon et al. [75] refined the prediction of Giannetti et al. [51] and experimentally and numerically found that the flow becomes oscillatory at $R e<1970$ and a steady-unsteady transition occurs in the range $1700<R e<1970$.

As summarized by Gómez et al. [54]: the flow structure of three-dimensional liddriven cavity are far more complicated compare to the two-dimensional flow. The most importrant three-dimensional flow features are the Taylor-Görtler like (TGL) vortices and corner eddies or end-wall vortices (EWV) in the flow field, primary eddy (PE), downstream secondary eddy (DSE), upstream secondary eddy (USE) and upstream upper eddy (UUE), as shown in figure 4.25 .

The objective of this section is to validate the complex three-dimensional flow. From DNS results, the three-dimensional base flow is obtained accurately by comparing to previous researches. Table 4.13 assembles the minimum and maximum velocity $u, v$ on the centerlines of the current research and the references values from previous studies. As the resolutions increased, the discrepancies between current research and the reference solution are smaller, for the mesh $96 \times 96 \times 96$, it is sufficient to capture the base flow. Figure 4.26 shows comparisons of normal velocities at the centerlines of the cubic lid-driven cavity at $R e=1000$, calculated on mesh $96 \times 96 \times 96$, against reference values.

\section{Linear instability analysis results}

Validation of the present three-dimensional global instability analysis algorithm has been provided by comparison against reference works, starting with those of Giannetti et al. [51] and Gómez et al. [54]. 10 eigenvalues are obtained using times-stepping method with $m=40$ dimensional Krylov subspace, time integration $\tau=0.6$. Table 4.14 demonstrates the comparison between the reference eigenvalues of Gómez et al. [54] and the 


\begin{tabular}{lcccccccccc}
\hline Method & $\mathrm{AR}$ & $N_{x}$ & $N_{y}$ & $N_{z}$ & $v_{\min }$ & $x$ & $v_{\max }$ & $x$ & $u_{\min }$ & $y$ \\
\hline SCM $^{\dagger}$ & $1: 1: 1$ & 96 & 96 & 96 & -0.4350 & 0.9096 & 0.2466 & 0.1091 & -0.2803 & 0.1242 \\
\hline FVM $^{*}$ & $1: 1: 1$ & 144 & 144 & 144 & -0.4333 & 0.9076 & 0.2456 & 0.1073 & -0.2792 & 0.1235 \\
\hline SEM & $1: 1: 1$ & 96 & 96 & 96 & -0.4350 & 0.9096 & 0.2466 & 0.1090 & -0.2803 & 0.1244 \\
\hline SEM & $1: 1: 1$ & 64 & 64 & 64 & -0.4348 & 0.9104 & 0.2466 & 0.1104 & -0.2800 & 0.1296 \\
\hline
\end{tabular}

Table 4.13: Comparison results of the minimum and maximum velocities on the centerlines $v(x, 0.5,0.5)$ and $u(0.5, y, 0.5)$ of the cubic lid-driven cavity at $R e=1000 . \mathrm{SCM}^{\dagger}$ spectral results from Albensoeder and Kuhlmann [2] and $\mathrm{FVM}^{*}$ finite volume method results from Gómez et al. [54], SEM represent current results from spectral element method.
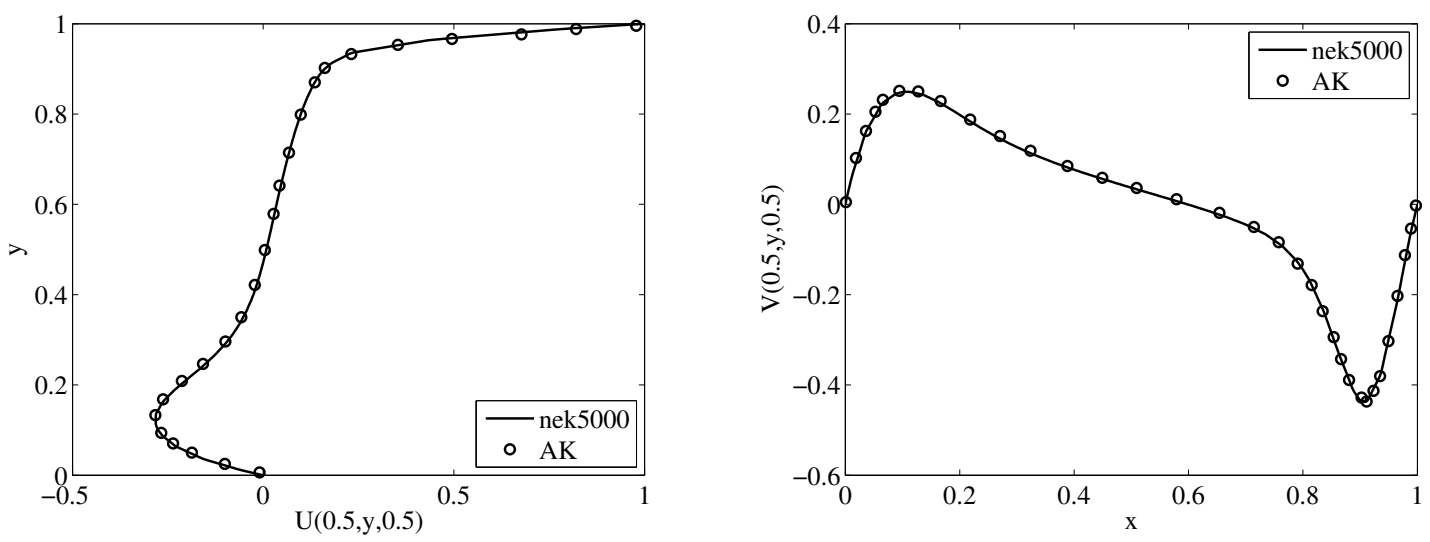

Figure 4.26: Normal velocity on the centerlines $(0.5, y, 0.5)$ and $(x, 0.5,0.5)$ in the cubic cavity for $L: W: D=1: 1: 1$ using a resolution $N_{x} \times N_{y} \times N_{z}=96 \times 96 \times 96$ at $R e=1000$. The symbol $(\diamond)$ represents the results of Albensoeder and Kuhlmann [2]. 


\begin{tabular}{cccc}
\hline & Mode I & Mode II & Mode III \\
\cline { 2 - 4 } & $\lambda_{r} \pm i \cdot \lambda_{i}$ & $\lambda_{r} \pm i \cdot \lambda_{i}$ & $\lambda_{r} \pm i \cdot \lambda_{i}$ \\
\hline Current results & $-0.1353 \pm i \cdot 0.299$ & $-0.1304 \pm i \cdot 0.488$ & $-0.1372 \pm i \cdot 0.000$ \\
\hline Gómez et al. [54] & $-0.1360 \pm i \cdot 0.285$ & $-0.1348 \pm i \cdot 0.485$ & $-0.1382 \pm i \cdot 0.000$ \\
\hline F. Giannetti et al.[51] & $-0.1356 \pm i \cdot 0.284$ & $-0.1377 \pm i \cdot 0.457$ & $-0.1453 \pm i \cdot 0.000$ \\
\hline
\end{tabular}

Table 4.14: Comparison of the three eigenvalues of the lid-driven cavity at $R e=1000$.

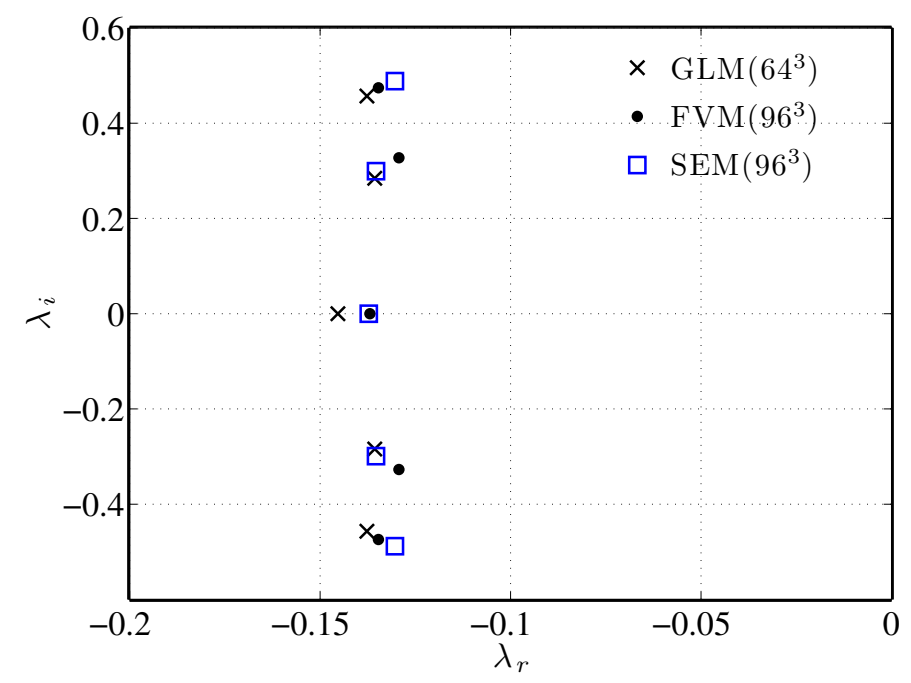

Figure 4.27: Spectrum of cubic lid-driven cavity at $R e=1000$ calculated by Giannetti et al. $[51](\mathrm{GLM}, \times)$ and Gómez et al. [54](FVM, •) and current method (SCM, $\square)$

present results, also the comparison can be checked in figure 4.27 , an overall good agreement between the location of the eigenvalues can be seen, while the differences can be explained by the different numerical methods employed. Figure 4.28 shows the eigenfunctions corresponding of two traveling modes $\lambda_{1}=-0.1353+i \cdot 0.299, \lambda_{2}=-0.1304+i \cdot 0.488$ and one stationary mode $\lambda_{3}=-0.1372+i \cdot 0.000$. At this Reynolds number, it is observed that the two traveling eigenvalues are related to the corresponding modes of the two dimensional flow, properly affected by the presence of the wall and thus the above-mentioned EWV structure, however, the existence of different n-cell symmetric and asymmetric families of linear modes with TGL structure may be inferred from the third mode. 


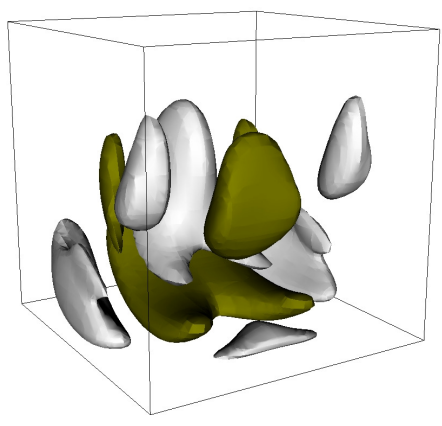

(a) $\hat{u}(x, y, z)$

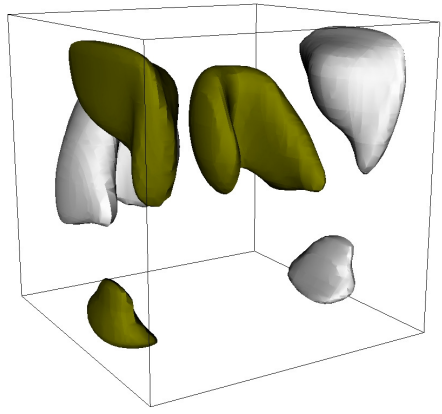

(d) $\hat{u}(x, y, z)$

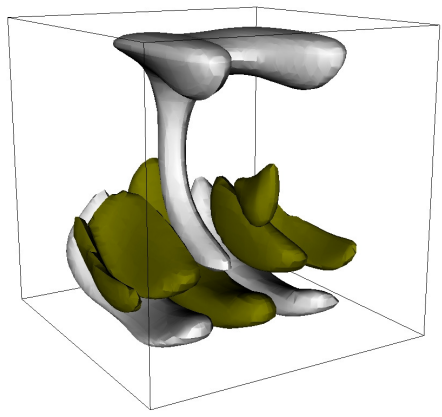

(g) $\hat{u}(x, y, z)$

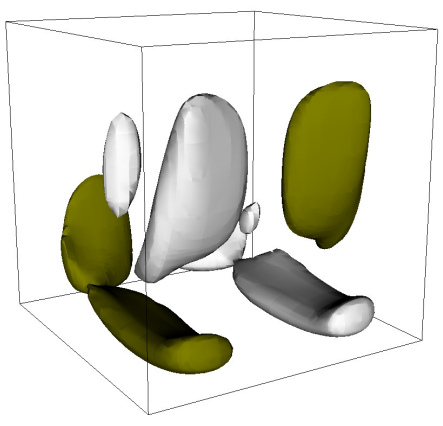

(b) $\hat{v}(x, y, z)$

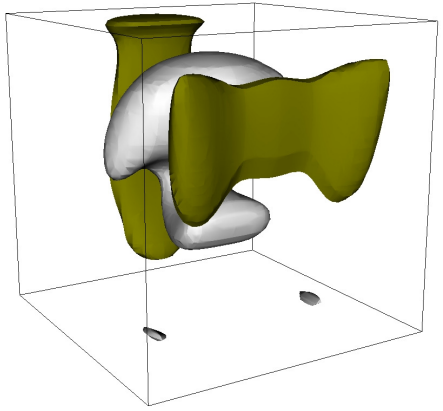

(e) $\hat{v}(x, y, z)$

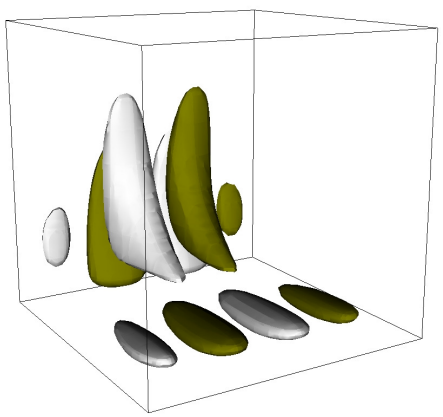

(h) $\hat{v}(x, y, z)$

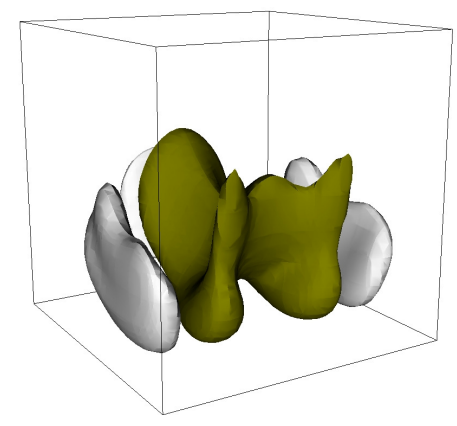

(c) $\hat{w}(x, y, z)$

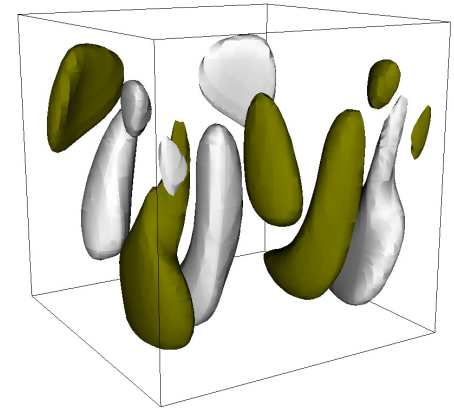

(f) $\hat{w}(x, y, z)$

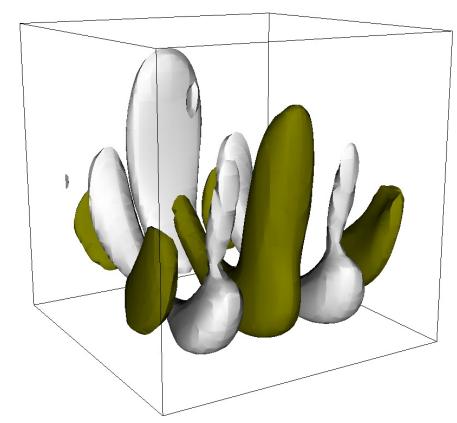

(i) $\hat{w}(x, y, z)$

Figure 4.28: (a)-(c)mode I: $-0.1353+i \cdot 0.299 \hat{u}(x, y, z)= \pm 0.6, \hat{v}(x, y, z)= \pm 0.8, \hat{w}(x, y, z)= \pm 0.8$ (d)-(f)mode II :-0.1304 $+i \cdot 0.488 \hat{u}(x, y, z)= \pm 0.6, \hat{v}(x, y, z)= \pm 0.6, \hat{w}(x, y, z)= \pm 1$ (g)(i)mode III: $-0.1372+i \cdot 0.000, \hat{v}(x, y, z)= \pm 0.8, \hat{v}(x, y, z)= \pm 0.8, \hat{w}(x, y, z)= \pm 1$ 


\section{Chapter 5}

\section{Three-dimensional wall-bounded open cavity flow}

In the previous chapter, the numerical methods have been validation by the cases of twodimensional and three-dimensional open flow. In this chapter, direct numerical simulations (DNS) have been performed at different Reynolds numbers to study the flow over threedimensional open cavity with lateral walls. Flow topology variations are presented. The numerical results are validated with the experiment results [31]. The characteristics of both direct and adjoint perturbation modes are studied and the regions of the structural sensitive to momentum forcing and mass injection are identified.

\subsection{Model description and parameters}

Numerical simulations have been performed to study the flow on a wall-bounded threedimensional open cavity with geometrical ratio $\mathrm{L}: \mathrm{W}: \mathrm{D}=6: 2: 1$, where $\mathrm{L}, \mathrm{W}$ and $\mathrm{D}$ are the length, width and depth of the cavity respectively. The origin Cartesian coordinate $(0,0,0)$ are fixed in the corner of the upstream of the cavity, $x, y, z$ are the direction of streamwise, transverse, spanwise, respectively.

DNS and instability analysis have been carried out over the results whilst increasing Reynolds number, defined as:

$$
R e=\frac{D U}{\nu}
$$

where $U$ is the freestream velocity and $\nu$ represents the dynamic viscosity.

Moreover, the incoming flow is laminar. Blasius velocity profile is implemented at the inlet boundary condition, in which subscripts 1 and 0 represents the position at the beginning of computational domain and at the upstream lip of the cavity, respectively. Reynolds number depending on the boundary layer displace thickness $\delta^{*}$ is defined as $R e_{\delta^{*}}=\frac{\delta^{*} * U}{\nu}$, the boundary layer momentum thickness is labeled as $\theta$, and the Reynolds number depending on the boundary layer displace thickness $\theta$ is defined as $\operatorname{Re}_{\theta}=\frac{\theta * U}{\nu}$.

Figure 5.1 shows the model and the parameters defined in the simulations. The numerical values employed for all these parameters are listed in table 5.1.

\subsection{Boundary conditions and initial conditions}

Because of the mesh employed was created using the Nek5000 utility genbox, it was composed by two sets of spectral element grids: velocity $\mathbf{u}$ are discretized on the Chebyshev Gauss Lobatto (CGL) grids, while pressure $p$ is resolved on the Chebyshev Gauss (CG) grids. This is called staggered grid technique (See in Appendix B), and it removes the need for pressure boundary conditions.

Figure 5.1 provide the boundaries defining the computational region, there are five different kinds of boundaries defining the computational domain in DNS: 


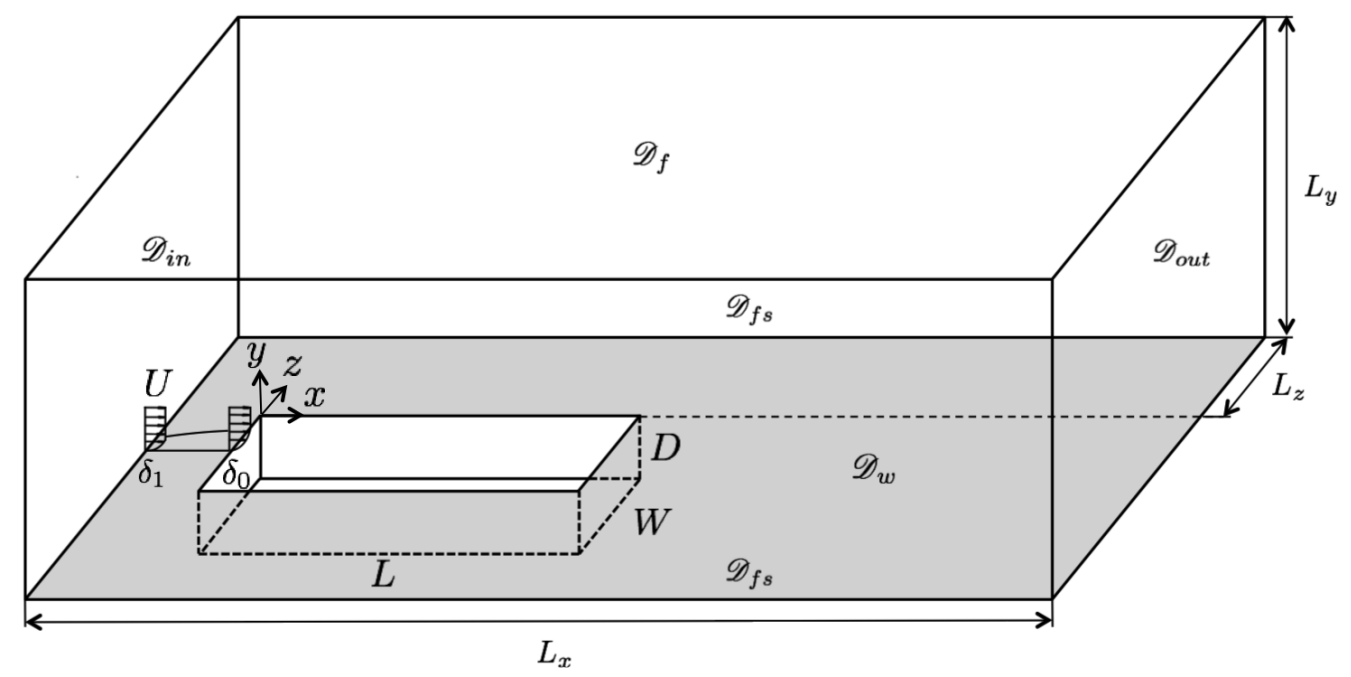

Figure 5.1: Computational configuration of full 3D open cavity

\begin{tabular}{ccccc}
\hline$R e$ & $\theta_{0}$ & $\operatorname{Re}_{\theta_{0}}$ & $\operatorname{Re}_{\delta_{0}^{*}}$ & $\theta_{1}$ \\
\hline 900 & 0.0313 & 28.341 & 73.007 & 0.057 \\
\hline 950 & 0.0305 & 29.117 & 75.008 & 0.056 \\
\hline 1000 & 0.0297 & 29.874 & 76.957 & 0.054 \\
\hline 1050 & 0.0290 & 30.612 & 78.857 & 0.053 \\
\hline 1100 & 0.0285 & 31.332 & 80.731 & 0.052 \\
\hline
\end{tabular}

Table 5.1: Parameters characterizing the inflow Blasius boundary layer of the three-dimensional open cavity with geometric ratio $L: W: D=6: 2: 1$. 
- Inlet $\mathscr{D}_{\text {in }}$ :

$$
\mathbf{u}=\left(u_{\text {blasius }}, v_{\text {blasius }}, 0\right)
$$

- Wall $\mathscr{D}_{w}$ :

$$
\mathbf{u}=(0,0,0)
$$

- Farfield $\mathscr{D}_{f}$

$$
u=U=1, \frac{\partial v}{\partial y}=0
$$

- Outlet $\mathscr{D}_{\text {out }}$

$$
\left(p-\frac{1}{R e} \nabla \mathbf{u}\right) \cdot \mathbf{n}=0
$$

- Sides of the computational domain $\mathscr{D}_{f s}$

$$
\mathbf{u} \cdot \mathbf{n}=0 ; \quad(\nabla \mathbf{u} \cdot t) \cdot \mathbf{n}=0
$$

While the perturbation boundary condition in linear stability analysis is defined as following:

- Inlet $\mathscr{D}_{i n}$ :

$$
\mathbf{u}=(0,0,0)
$$

- Wall $\mathscr{D}_{w}$ :

$$
\mathbf{u}=(0,0,0)
$$

- Farfield $\mathscr{D}_{f}$

$$
u=0, \frac{\partial v}{\partial y}=0
$$

- Outlet $\mathscr{D}_{\text {out }}$

$$
\left(p-\frac{1}{R e} \nabla \mathbf{u}\right) \cdot \mathbf{n}=0
$$

- Sides of the computational domain $\mathscr{D}_{f s}$

$$
\mathbf{u} \cdot \mathbf{n}=0 ; \quad(\nabla \mathbf{u} \cdot t) \cdot \mathbf{n}=0
$$

\subsubsection{Domain and grid validations}

It is critical importance that results of the open flow be independent of the size of the computational domain and the boundary treatment. Colonius et al. [29] has studied boundary conditions for the direct computation, it shows that if the boundary is treated improperly, the physical instability will be difficult to identify with the spurious reflections of waves, which leads to self-forcing of the flow, also the improper domain size will destroy flow structure inside the cavity. So before the parameters studies, domain validation analysis needs to be performed, the corresponding relative percentage error is introduced to quantify the discrepancy between each computational domain, it is defined as below:

$$
\epsilon_{j}=\left|\frac{u_{j}-u_{r e}}{u_{r e}}\right| \times 100,
$$


5. Three-dimensional wall-bounded open cavity flow

\begin{tabular}{rcccccc}
\hline Domain & $|u|_{\max }$ & $x$ & $\epsilon(\%)$ & $|w|_{\max }$ & $z$ & $\epsilon(\%)$ \\
\hline$L_{z}=4 D$ & 0.248183 & 5.862 & 0.02136 & 0.014882 & 0.193 & 0.09385 \\
\hline$L_{z}=8 D$ & 0.248134 & 5.862 & 0.00161 & 0.014895 & 0.193 & 0.00671 \\
\hline$L_{z}=12 D$ & 0.248130 & 5.862 & - & 0.014896 & 0.193 & - \\
\hline
\end{tabular}

Table 5.2: The maximum absolute value of $|u|_{\max }$ at the location of $(x,-0.5,0.5)$ and $|w|_{\max }$ at the location of $(5,-0.5, z)$ inside cavity at $R e=1000$.

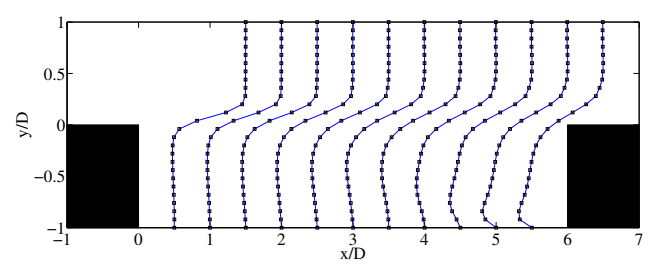

(a)

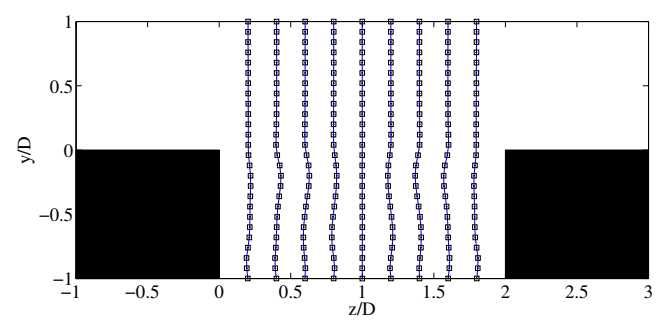

(b)

Figure 5.2: (a) Comparison of the streamwise velocity $u$ in $x-y$ plane at $z=0.5$ in three difference spanning of computational domains at $R e=1000$. (b) Comparison of the spanwise velocity $w$ in $\mathrm{y}$ z plane at $x=5$ in the different lateral computational domain sizes at $R e=1000$. The streamwise velocity of $L_{z}=4 D, L_{z}=8 D$ and $L_{z}=12 D$ are plotted by $-\bullet, \square$, respectively.

where $u_{r e}$ yields any field value discretized on the largest domain size.

We have run the three-dimensional cavity case with three different computational domain size $L_{z}=4 \mathrm{D}, 8 \mathrm{D}, 12 \mathrm{D}$ and $L_{y}=3 \mathrm{D}, 5 \mathrm{D}$ and $7 \mathrm{D} . u_{r e}$ is any flow variable probed from the largest domain size $L_{z}=12 D$ and $L_{y}=7 D$.

Table 5.2 shows the relative percentage errors of three difference spanning of computational domains. The relative errors show that the result of the flow field is independent of the computational domain size since $L_{z}=4$, the streamwise velocity from domain size $L z=4$ are consistent with the other larger domains $L z=8$ and $L z=12$, it is enough to accurately simulate the cuboid cavity flow. Further, spatial visualization of the velocity component $u$ in $x-y$ plane at $z=0.5$ and $w$ in $y-z$ plane at $x=5$ are shown in figure 5.2. The discretized velocity components are in good agreements along three different spanning of computational domains. Considering the cost of the computational, $L_{z}=4$ is chosen as the side domain size.

The evaluations of the height of computational domain $L_{y}$ have been carried out through review of previous studies. In the work of Citro et al. [26], simulations were performed on an open cavity with a length to depth ratio $L / D=1$. The computational domain height is $L_{y}=0.5 \mathrm{D}$, and the initial boundary layer thickness displacement $\delta^{*}=0.0169$ 


\begin{tabular}{ccc}
\hline Height & $u(L / 2,0, W / 2)$ & $\epsilon(\%)$ \\
\hline$L_{y}=3 D$ & -0.0828 & 1.222 \\
\hline$L_{y}=5 D$ & -0.0820 & 0.244 \\
\hline$L_{y}=8 D$ & -0.0818 & - \\
\hline
\end{tabular}

Table 5.3: Comparison of the streamwise velocity $u(x, y)$ with three different height domain in the probe point $(x=L / 2, y=0, z=W / 2)$ of the cavity.

\begin{tabular}{rc}
\hline Domain $[x] \times[y] \times[z]$ & Elements \\
\hline$[-1,0] \times[0,5] \times[-4,0]$ & $4 \times 12 \times 8$ \\
\hline$[-1,0] \times[0,5] \times[0,2]$ & $4 \times 12 \times 8$ \\
\hline$[-1,0] \times[0,5] \times[2,6]$ & $4 \times 12 \times 8$ \\
\hline$[0,6] \times[0,5] \times[-4,0]$ & $16 \times 12 \times 8$ \\
\hline$[0,6] \times[0,5] \times[0,2]$ & $16 \times 12 \times 8$ \\
\hline$[0,6] \times[0,5] \times[2,6]$ & $16 \times 12 \times 8$ \\
\hline$[6,12.2] \times[0,5] \times[-4,0]$ & $11 \times 12 \times 8$ \\
\hline$[6,12.2] \times[0,5] \times[0,2]$ & $11 \times 12 \times 8$ \\
\hline$[6,12.2] \times[0,5] \times[2,6]$ & $11 \times 12 \times 8$ \\
\hline$[0,6] \times[-1,0] \times[0,2]$ & $16 \times 6 \times 8$ \\
\hline
\end{tabular}

Table 5.4: Mesh details of the cuboid open cavity.

at the leading edge of the cavity. The ratio between $L_{y} / \delta^{*}=0.5 / 0.0169 \sim 30$ was proved as a sufficient domain size. It is worth to note the instabilities of this type flow are confined in the cavity, centrifugal instability, it has a very little influence of the outside domain.

Following above reference, height validations are carried on $L_{y}=3 \mathrm{D}, 5 \mathrm{D}$ and $7 \mathrm{D}$ with the initial boundary layer thickness displacement $\delta^{*}=0.0734$. The streamwise velocity $u$ at $(3,0,1)$ are probed in these three different computational domain at $R e=1000$, as shown in table 5.3. The computational domain with $L_{y}=5 \mathrm{D}$ is adequate to guarantee the open flow simulation. Moreover, the effect of the height domain on instability analysis are documented in section 5.7.

The mesh is generated by the spectral element method (genbox) that is part of the Nek5000 package, the refined mesh is supplied in the boundary layer and near the wall in order to make sure accurately spatial analog. The computational domain is split into 10 blocks. The shallow cavity is in the one block with $16 \times 6 \times 8$ elements, the length of first elements which attach the wall and shear layer are fixed to 0.16, it is approximate to the twice of the displacement thickness $\delta_{0}^{*}=0.086$ of the boundary layer, the polynomial order is tested in $n=5,7,9$ with a constant element distribution. The details of mesh information are documented in figure 5.3 and table 5.4. The validations of the grid convergence are in figure 5.4, it is evident that the grid with polynomial order $n=5$ is low-fidelity to interpret the flow field, but the grid with polynomial order $n=7$ is good agreement with higher order $n=9$, hence the mesh with polynomial order $n=7$ is choice for the sake of less computational cost. 


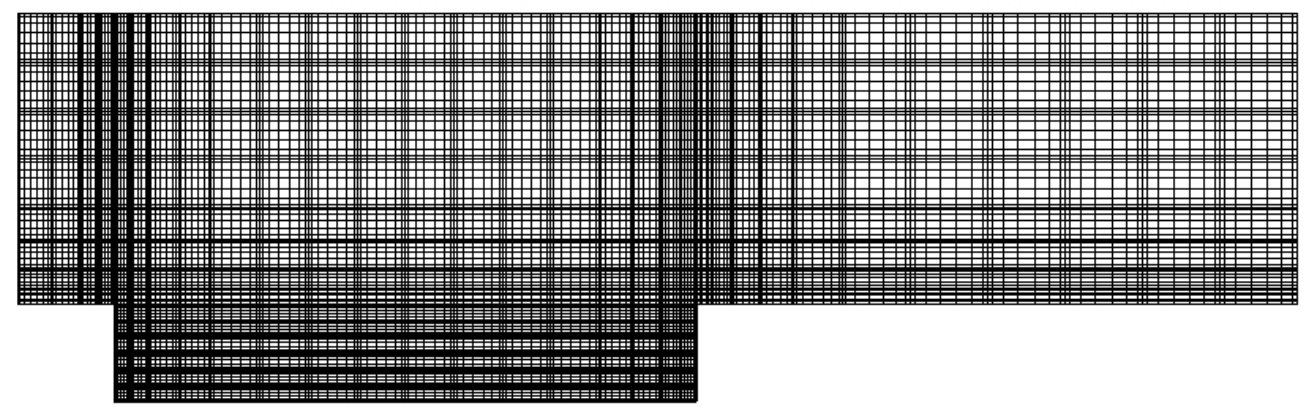

(a) $x-z$ plane

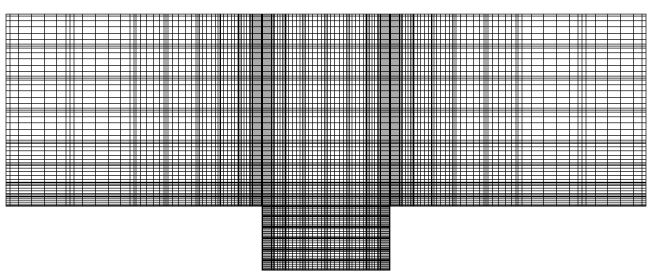

(b) $y-z$ plane

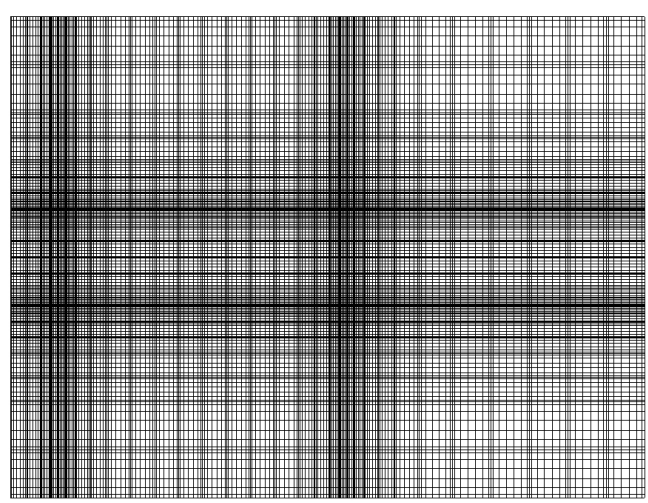

(c) $x-y$ plane

Figure 5.3: The grid detail for computation

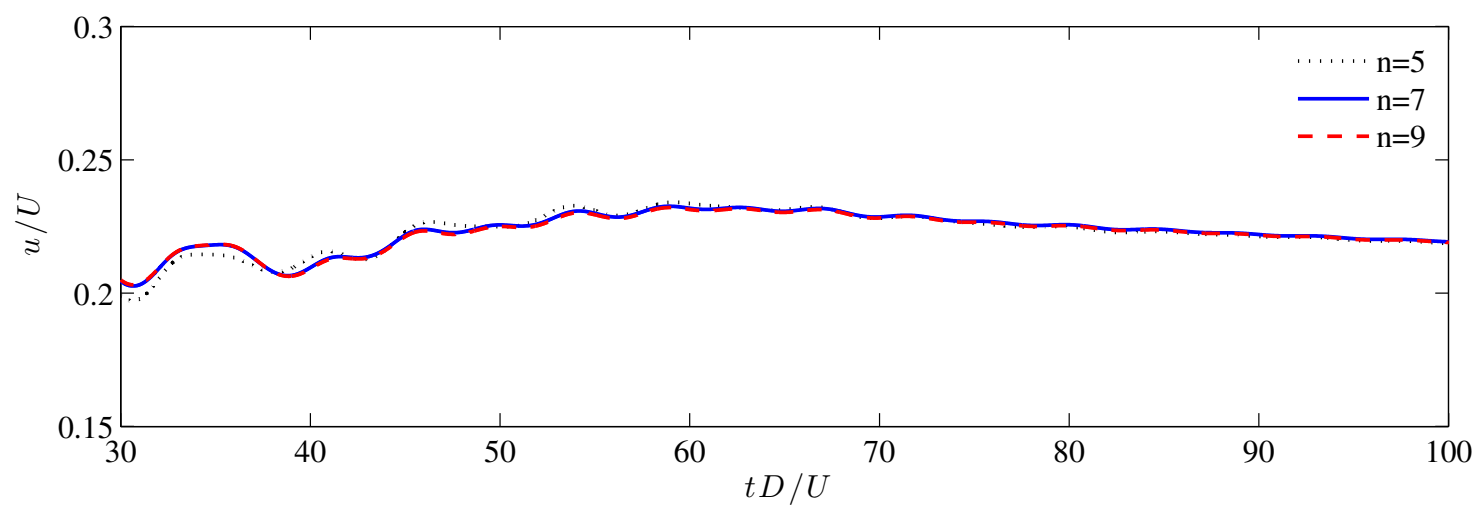

Figure 5.4: Validation of the time history of the streamwise velocity $u(x, y, z)$ in the center of the cavity $(3,0,1)$ at $R e=1000$ with three different polynomial orders. 
5.3. Tri-Global linear instability of three-dimensional open cavity flow

\subsection{Tri-Global linear instability of three-dimensional open cavity flow}

The flow over the three-dimensional cavity is analyzed here with an increasing of Reynolds number. Our interest is to highlight the variation of the flow topology and instability characteristics as the flow conditions transit from subcritical to supercritical condition. According to the previous studies [77], the critical Reynolds number of this kind of flow with cavity aspect ratio $L: W: D=6: 2: 1$ is revealed at these conditions: $1100<R e_{c r}<1150$, this condition is higher than $2 \mathrm{D}$ and $3 \mathrm{D}$ open cavity with the same length to depth ratio. In the study of Sun et al. [110], they pointed the stability diagram of the 2D open cavity flow for $L / D=6$, the critical Reynolds number is around $R e_{c r} \sim 790$ at low Mach number.

A more complex flow structure and unsteadiness comes with a higher Reynolds number. In order to thorough understand the instability properties, subcritical flow conditions $(R e=900, R e=950, R e=1000$ and $R e=1050)$ are considered here using DNS and TriGlobal linear instability analysis.

\subsubsection{Case 1: $R e=900$}

\section{Base Flow}

The DNS is carried out on incompressible flow over the cavity at $R e=900$. The simulation is initialized by a Blasius velocity profile above the cavity, flow undergoes a transition phrase and becomes steady gradually, and ultimately convergences to a steady state.

Figure 5.5 shows the time-history of the streamwise velocity $u(3,0,1)$. The other two components of the velocity have the similar behavior as streamwise velocity. Intuitively, the amplitude of the velocity oscillation decrease considerably, steamwise velocity $u(x, y, z)$ converges to a constant value ultimately.

Time evolution of kinetic energy $E$ residual is calculated by the equation (3.6). The kinetic energy $\mathrm{E}$ is defined as

$$
E=\sum^{N}\left(\frac{1}{2}\left(u^{2}+v^{2}+w^{2}\right)\right),
$$

where $\mathrm{N}$ is the discretization points of the computational domain.

Figure 5.6 shows the convergence history of kinetic energy. The numerical residuals is identified as being the stationary least damped perturbation. After a short initial transient, exponential decay of residuals comes out, this decay purses at the same rate for almost three decades. So the numerical solution is attracted by this steady large damping rate disturbance.

A visualization of the base flow structures are presented in figure 5.7(a). Spanwise velocity $w(x, y, z)$ is shown with a isosurface levels are \pm 0.01 . Antisymmetric structure are identified with a mainly distribution located in the rear of the cavity. Figure 5.7(b) plots the negative streamwise velocity (blue color) and the streamwise vorticity (grey color). The negative streamwise velocity is relate to the recirculation zone in the cavity. Except the rear vortex inside of cavity, a pair of edge vortices are generated along cavity edge due to the small difference in pressure between the outside and inside of the cavity. 


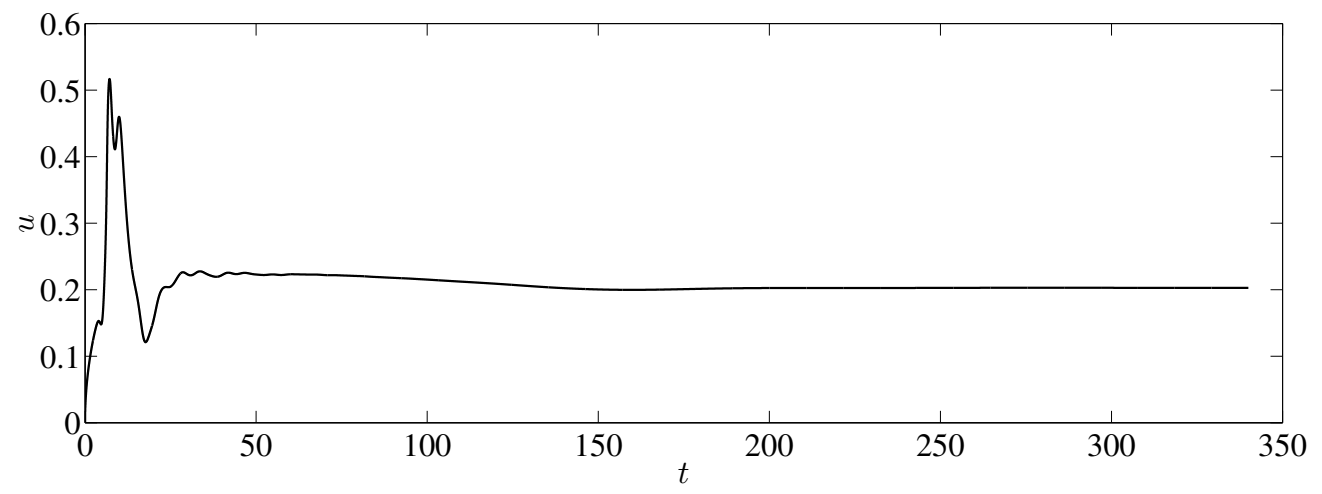

Figure 5.5: Time trace of streamwise velocity $u(3,0,1)$ and the details of the signal for the cuboid cavity at $R e=900$.

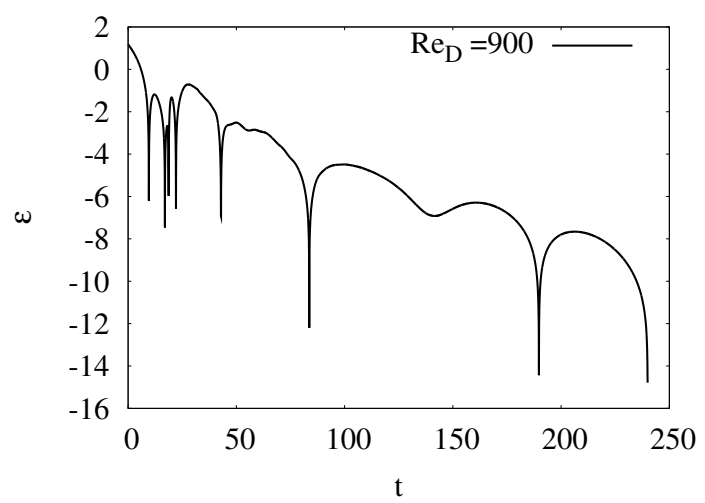

Figure 5.6: (a) Time evolution of kinetic energy residual of wall-bounded three-dimensional open cavity at $R e=900$. (b) Eigen-spectrum of obtained global modes at $R e=900$.

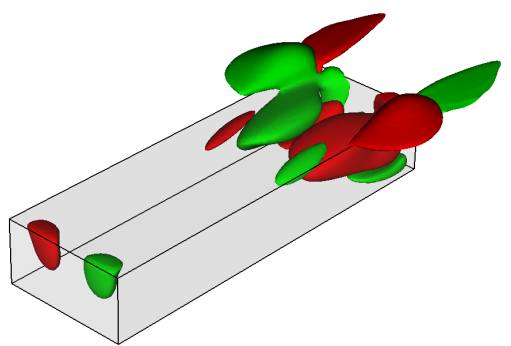

(a) $w(x, y, z)$

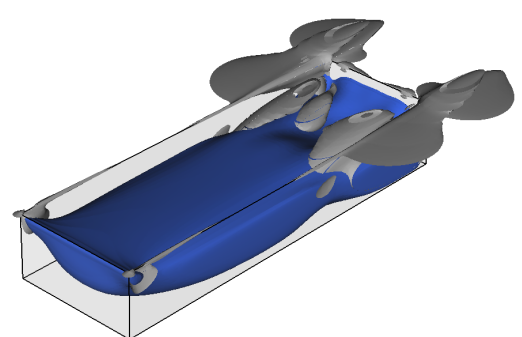

(b)

Figure 5.7: (b) Contour of the spanwise velocity $w(x, y, z)$ of the cuboid cavity at $R e=900$, isosurface level are $w(x, y, z)= \pm 0.01$. (c) Grey isosurfaces are streamwise vorticity $\omega_{x}= \pm 0.15$, the blue isosurfaces are negative streamwise velocity $u(x, y, z)$. 


\begin{tabular}{cc}
\hline Mode & $\lambda_{r} \pm \mathrm{i} \cdot \lambda_{i}$ \\
\hline CS & $-0.0136 \pm \mathrm{i} \cdot 0.0000$ \\
\hline CT & $-0.0198 \pm \mathrm{i} \cdot 0.0340$ \\
\hline CT1 & $-0.0310 \pm \mathrm{i} \cdot 0.0810$ \\
\hline CT2 & $-0.0380 \pm \mathrm{i} \cdot 0.0924$ \\
\hline CT3 & $-0.0424 \pm \mathrm{i} \cdot 0.1016$ \\
\hline CT4 & $-0.0409 \pm \mathrm{i} \cdot 0.0310$ \\
\hline
\end{tabular}

Table 5.5: The first six leading eigenvalues of the cuboid cavity at $R e=900$.

\section{Linear Instability Analysis}

TriGlobal instability analysis has been employed in this obtained base flow. Krylov subspace dimensions $m=60$ is performed for convergence of the 11 eigenmodes, the convergence criterion for the Arnoldi iteration is based on a tolerance of $10^{-6}$. From the residual of the DNS results in figure 5.6, the most of kinetic energy contains in the steady mode, the time integration $\tau=0.8$ make sure there is no aliasing phenomenon for the instability analysis.

Table 5.5 lists the calculated leading eigenvalues. For convenience, they are denoted as: centrifugal steady modes (CS), centrifugal traveling modes (CT) and centrifugal modes CT1, CT2, CT3, CT4 by frequency. The least stable eigenmode at this flow condition is the stationary eigenmode CS, with a largest damping ratio $\lambda_{c s}=-0.0136$. Here circular frequency $\lambda_{i}$ have been transferred using following equation,

$$
f=\frac{\lambda_{i}}{2 \pi} .
$$

Centrifugal traveling modes CT, CT1, CT2, CT3, CT4 have small frequencies $f=0.005-$ 0.016 .

Figure 5.8 shows the obtained global eigenvalues corresponding to the centrifugal modes. The leading eigenmode is a steady global mode. The spatial structure of each eigemode is visualized in figure 5.9. Spatial structures of three components of CS mode reveal the antisymmetric property of this instability, which associates to the primary vortices. Moreover, streamwise perturbation has a long tail along the downstream of the cavity. From the observation, CT, CT2 and CT3 are also the antisymmetric modes. Whereas, CT1 and CT4 are symmetric eigenmodes. All the global modes are confined inside cavity with various structures 